\title{
Parton distributions and lattice QCD calculations: a community white paper
}

Huey-Wen Lin ${ }^{1,2}$, Emanuele R. Nocera ${ }^{3,4}$, Fred Olness ${ }^{5}$, Kostas Orginos ${ }^{6,7}$, Juan Rojo ${ }^{8,9}$ (editors), Alberto Accardi ${ }^{7,10}$, Constantia Alexandrou ${ }^{11,12}$, Alessandro Bacchetta ${ }^{13}$, Giuseppe Bozzi ${ }^{13}$, Jiunn-Wei Chen ${ }^{14}$, Sara Collins ${ }^{15}$, Amanda Cooper-Sarkar ${ }^{16}$, Martha Constantinou ${ }^{17}$, Luigi Del Debbio ${ }^{4}$, Michael Engelhardt ${ }^{18}$, Jeremy Green ${ }^{19}$, Rajan Gupta $^{20}$, Lucian A. Harland-Lang ${ }^{3,21}$, Tomomi Ishikawa ${ }^{22}$,

Aleksander Kusina ${ }^{24}$, Keh-Fei Liu ${ }^{25}$, Simonetta Liuti26,27, Christopher Monahan ${ }^{28}$, Pavel Nadolsky ${ }^{5}$, Jian-Wei Qiu ${ }^{7}$, Ingo Schienbein ${ }^{23}$, Gerrit Schierholz ${ }^{29}$, Robert S. Thorne ${ }^{21}$, Werner Vogelsang ${ }^{30}$, Hartmut Wittig ${ }^{31}$, C.-P. Yuan ${ }^{1}$, and James Zanotti ${ }^{32}$

${ }^{1}$ Department of Physics and Astronomy, Michigan State University, East Lansing, MI 48824, USA

${ }^{2}$ Department of Computational Mathematics, Science and Engineering, Michigan State University, East Lansing, MI 48824, USA

${ }^{3}$ Rudolf Peierls Centre for Theoretical Physics, 1 Keble Road, University of Oxford, OX1 3NP Oxford, United Kingdom

${ }^{4}$ Higgs Centre for Theoretical Physics, School of Physics and Astronomy, University of Edinburgh, EH9 3FD, UK

${ }^{5}$ Department of Physics, Southern Methodist University, Dallas, TX 75275, USA

${ }^{6}$ Physics Department, College of William and Mary, Williamsburg, VA 23187, USA

${ }^{7}$ Thomas Jefferson National Accelerator Facility, Newport News, VA 23606, USA

${ }^{8}$ Department of Physics and Astronomy, VU University Amsterdam,

De Boelelaan 1081, NL-1081, HV Amsterdam, The Netherlands

${ }^{9}$ Nikhef, Science Park 105, NL-1098 XG Amsterdam, The Netherlands

${ }^{10}$ Hampton University, Hampton, VA 23668, USA

${ }^{11}$ Department of Physics, University of Cyprus, P.O. Box 20537, 1678 Nicosia, Cyprus

${ }^{12}$ Computation-based Science and Technology Research Research,

The Cyprus Institute, 20 Kavafi Str., Nicosia 2121, Cyprus

${ }^{13}$ Dipartimento di Fisica, Università degli Studi di Pavia, and INFN, Sezione di Pavia, 27100 Pavia, Italy

${ }^{14}$ Department of Physics, Center for Theoretical Sciences, and Leung Center for Cosmology and Particle Astrophysics, National Taiwan University, Taipei, Taiwan 106

${ }^{15}$ Institute for Theoretical Physics, Universität Regensburg, D-93040 Regensburg, Germany

${ }^{16}$ Department of Physics, University of Oxford,

Denys Wilkinson Building, 1 Keble Road, OX1 3RH Oxford, United Kingdom

17 Department of Physics, Temple University, Philadelphia, PA 19122, USA

${ }^{18}$ Department of Physics, New Mexico State University, Las Cruces, NM 88003-8001, USA

${ }^{19}$ NIC, Deutsches Elektronen-Synchrotron, 15738 Zeuthen, Germany

${ }^{20}$ Los Alamos National Laboratory, Theoretical Division, T-2, Los Alamos, NM 87545, USA

21 Department of Physics and Astronomy, University College London, WC1E 6BT, United Kingdom

${ }^{22}$ T. D. Lee Institute, Shanghai Jiao Tong University, Shanghai, 200240, P. R. China

${ }^{23}$ Laboratoire de Physique Subatomique et de Cosmologie, Universitè Grenoble-Alpes, CNRS/IN2P3, 53 avenue des Martyrs, 38026 Grenoble, France.

${ }^{24}$ Institute of Nuclear Physics Polish Academy of Sciences, PL-31342 Krakow, Poland

${ }^{25}$ Department of Physics and Astronomy, University of Kentucky, Lexington, KY 40506, USA

${ }^{26}$ Physics Department, University of Virginia, 382 MCormick Road, Charlottesville, VA 22904, USA

${ }^{27}$ Laboratori Nazionali di Frascati, INFN, Frascati, Italy

${ }^{28}$ Institute for Nuclear Theory, University of Washington, Seattle, WA 98195, USA

${ }^{29}$ Deutsches Elektronen-Synchrotron DESY, 22603 Hamburg, Germany

${ }^{30}$ Institute for Theoretical Physics, Tübingen University, D-72076 Tübingen, Germany

${ }^{31}$ PRISMA Cluster of Excellence and Institute for Nuclear Physics Johannes Gutenberg University of Mainz, Johann-Joachim-Becher-Weg 45, 55128 Mainz, Germany

${ }^{32}$ CSSM, Department of Physics, The University of Adelaide, Adelaide, SA, Australia 5005

Preprint numbers:

DESY 17-185, IFJPAN-IV-2017-19, INT-PUB-17-042, MSUHEP-17-017, Nikhef-2017-047, OUTP-17-15P, SMU-HEP-17-08. 


\begin{abstract}
In the framework of quantum chromodynamics (QCD), parton distribution functions (PDFs) quantify how the momentum and spin of a hadron are divided among its quark and gluon constituents. Two main approaches exist to determine PDFs. The first approach, based on QCD factorization theorems, realizes a QCD analysis of a suitable set of hard-scattering measurements, often using a variety of hadronic observables. The second approach, based on first-principle operator definitions of PDFs, uses lattice QCD to compute directly some PDF-related quantities, such as their moments. Motivated by recent progress in both approaches, in this document we present an overview of lattice-QCD and globalanalysis techniques used to determine unpolarized and polarized proton PDFs and their moments. We provide benchmark numbers to validate present and future lattice-QCD calculations and we illustrate how they could be used to reduce the PDF uncertainties in current unpolarized and polarized global analyses. This document represents a first step towards establishing a common language between the two communities, to foster dialogue and to further improve our knowledge of PDFs.
\end{abstract}

\title{
Contents
}

1 Introduction and motivation $\quad 3$

2 Theory overview $\quad 5$

2.1 Parton distribution functions . . . . . . . . . . . . . . . . . . . . 5

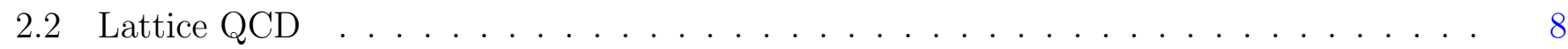

2.2.1 Systematic uncertainties . . . . . . . . . . . . . . . . . . 8

2.2.2 Mellin moments of PDFs from lattice QCD . . . . . . . . . . . . . . . 10

2.2.3 The $x$-dependence of PDFs from lattice QCD . . . . . . . . . . . . . . . . . . 12

2.3 Global PDF fits . . . . . . . . . . . . . . . . . . . . . . . . . . . . 18

2.3.1 General framework . . . . . . . . . . . . . . . . . . . . . . . 18

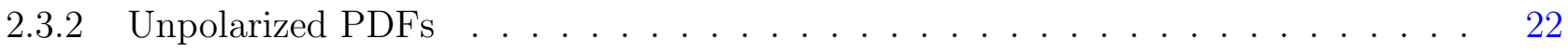

2.3.3 Polarized PDFs . . . . . . . . . . . . . . . . . . . . . . . 25

3 Benchmarking PDF moments $\quad 31$

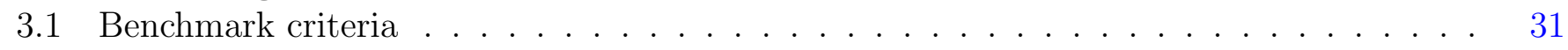

3.1.1 Benchmark quantities . . . . . . . . . . . . . . . . . . 31

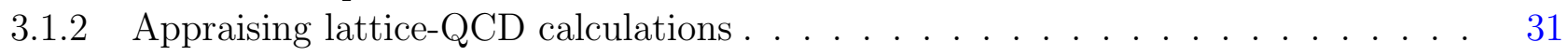

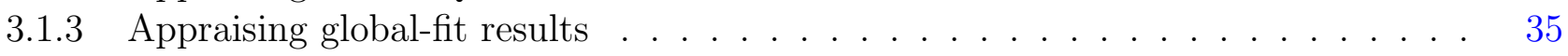

3.2 Comparing lattice-QCD and global-fit benchmark moments . . . . . . . . . . . . . . 39

4 Improving PDF fits with lattice-QCD calculations 42

4.1 Impact of lattice calculations of PDF moments . . . . . . . . . . . . . . . . . . . . . . . . . . . . . . . . . .

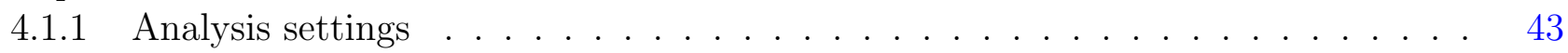

4.1 .2 Bayesian reweighting analysis . . . . . . . . . . . . . . . . . . . . . . . . 44

4.1.3 Hessian profiling analysis . . . . . . . . . . . . . . . . . . . . 50

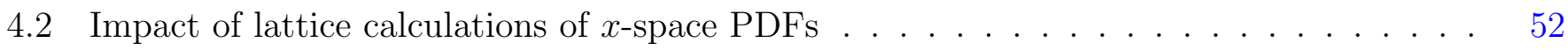

4.3 Discussion . . . . . . . . . . . . . . . . . . . . . . . . . 54

$\begin{array}{llr}5 & \text { Outlook } & 55\end{array}$

$\begin{array}{ll}\text { A Definition of the PDF moments } & 57\end{array}$

$\begin{array}{lll}\text { B PDF moments from lattice QCD } & 58\end{array}$

C PDF fit results for higher moments $\quad 65$ 


\section{Introduction and motivation}

The detailed understanding of the inner structure of nucleons is an active research field with phenomenological implications in high-energy, hadron, nuclear and astroparticle physics. Within quantum chromodynamics (QCD), information on this structure - specifically on how the nucleon's momentum and spin are divided among quarks and gluons — is encoded in parton distribution functions (PDFs).

There exist two main methods to determine PDFs.

The first method is the global QCD analysis [1-10]. It is based on QCD factorization of physical observables, i.e. the fact that a class of hard-scattering cross-sections can be expressed as a convolution between short-distance, perturbative, matrix elements and long-distance, nonperturbative, PDFs. By combining a variety of available hard-scattering experimental data with state-of-the-art perturbative calculations, complete PDF sets, including the gluon and various combinations of quark flavors, are currently determined for protons, in both the unpolarized [11-15] and polarized [16-19] case.

Recent progress in global QCD analyses has been driven, on the one hand, by the increasing availability of a wealth of high-precision measurements from Jefferson Lab, HERA, RHIC, the Tevatron and the LHC and, on the other hand, by the advancement in perturbative calculations of QCD and electroweak (EW) higher-order corrections. Parton distributions are now determined with unprecedented precision, in many cases at the few-percent level. A paradigmatic illustration of this progress is provided by both the unpolarized and polarized gluon PDFs, which were affected by rather large uncertainties until recently, due to the limited experimental information available. In the unpolarized case, the gluon PDF is now constrained quite accurately from small to large $x$ thanks to the inclusion of processes such as inclusive deep-inelastic scattering (DIS) [20], D-meson production [21,22], the transverse momentum of $Z$ bosons [23], inclusive jet production [24], and top-quark pair distributions [25,26]. In the polarized case, the gluon PDF is now constrained from double spin-asymmetries for high- $p_{T}$ jet and pion production in proton-proton collisions [16,27], although only in the medium-to-large $x$ region.

The second method is lattice $Q C D[28,29]$. It is based on the direct computation of the QCD path integral in a discretized finite-volume Euclidean space-time, providing a suitable ultraviolet cutoff. To connect with experimental measurements, extrapolations to the continuum and infinite-volume limits are necessary so that any cut-off dependence and finite-volume effects, respectively, are removed. Lattice-QCD calculations require minimal external input: one needs only to set the hadronic scale

$\Lambda_{\mathrm{QCD}}$ and the values of the quark masses. For calculations relevant to low-energy hadron structure, this means setting the up, down and strange quark masses, which is usually done using the pion and kaon masses as external inputs. The overall hadronic scale can be set using well-determined baryon masses such as that of the $\Omega$ baryon. A variety of QCD quantities can then be computed using lattice QCD, including moments of PDFs or of certain quark flavor PDF combinations.

Early lattice-QCD attempts to determine the proton PDFs were limited by the available computational resources and various technical challenges, with most results restricted to the first few moments of nonsinglet PDFs at relatively large (unphysical) quark masses. Overcoming these limitations, recent progress has been mostly driven by advances in two main areas. First, by improved systematic control (physical pion mass, excited-state contamination, large volumes) for quantities such as the nucleon matrix elements corresponding to the low moments of PDFs. Second, by the development of novel strategies for the computation of the first few moments [30-32], the determination of more challenging quantities such as gluon and flavor-singlet matrix elements, and for the direct calculation of the Bjorken- $x$ dependence of PDFs [33-36].

These developments have pushed lattice-QCD calculations to the point where, for the first time, it is possible to provide information on the PDF shape of specific flavor combinations, both for quarks and for antiquarks, and where meaningful comparisons with global fits can be made. Indeed, one of the main motivations for these lattice-QCD efforts is to achieve a sufficient accuracy to constrain the PDFs obtained from global analyses. 
Despite these developments in both the global QCD analysis and lattice-QCD methods, interplay between the two - and communication between the respective communities of physicists - have been rather limited so far. This situation led some of us to organize the first workshop on Parton Distributions and Lattice Calculations in the LHC Era (PDFLattice2017), which took place in Balliol College, University of Oxford, in March 2017. ${ }^{1}$ The main goal of this workshop was to establish a common ground and language for discussions between the two communities. In addition, we aimed to carry out a first quantitative exploration of how PDF fits can be exploited to benchmark existing and future lattice calculations, and of how lattice-QCD calculations could be used to improve global PDF fits. In this context, some of the questions that were addressed during this workshop included the following.

- What information from PDF fits is relevant to constrain, test, or validate lattice calculations?

- What PDF-related quantities are most compelling to compute in lattice QCD in terms of phenomenological relevance?

- What accuracy do we need from lattice quantities in order to have a significant impact on global PDF fits?

- What information does lattice QCD provide on the shape (Bjorken- $x$ dependence) of the PDFs? Which specific PDF moments can be computed?

- How do we consistently quantify the systematic uncertainties in lattice-QCD calculations?

- To what extent do available lattice results agree with the results of global PDF fits? Is there a tension between global PDF fits, PDF fits based on reduced datasets, and PDF calculations from the lattice?

- What is the accuracy that can be expected from lattice-QCD calculations in the near and medium future? What will be their constraining power on PDFs?

This white paper summarizes the joint effort between the two communities to address some of these questions, and follows up on the very fruitful discussions and interactions that took place both during the workshop and in the subsequent months. While this document does not represent the final word on this topic, it provides a solid starting point for further collaborative efforts, and should facilitate smooth interactions between the two communities in the future.

The outline of this white paper is the following. In Sec. 2 we review the global QCD analysis and lattice-QCD methods for the determination of polarized and unpolarized PDFs. In Sec. 3 we present state-of-the-art benchmarks for selected PDF moments between the most recent lattice-QCD calculations and global QCD analyses. In Sec. 4 we quantitatively assess the impact that lattice calculations of PDF-related quantities could have on unpolarized and polarized global analyses, assuming different scenarios for the uncertainties in the lattice-QCD calculations. In Sec. 5 we conclude and discuss future interactions between the global-analysis and lattice-QCD communities. In Appendix A we summarize the conventional notation adopted in this document for the definition of the PDF moments; in Appendix B we compile bibliographical tables for existing lattice-QCD calculations of PDF moments; and in Appendix $\mathrm{C}$ we collect some additional results of PDF moments from global QCD analyses.

\footnotetext{
${ }^{1}$ http://www.physics.ox.ac.uk/confs/PDFlattice2017/index.asp
} 


\section{Theory overview}

In this section we summarize the theoretical background that underlies lattice-QCD calculations of PDF-related quantities, on the one hand, and phenomenological fits of PDFs, on the other hand. We first review the general framework in which unpolarized and polarized PDFs are defined, then we present the available lattice-QCD and global-fit approaches to determine them. The discussion is restricted to the information required to connect the lattice-QCD and global-fit methods. We have devoted particular attention to ensuring a unified and consistent notation between the two. An extended treatment of the subjects discussed in this section can be found in dedicated reviews. We refer the interested reader to Refs. [28, 29] for lattice QCD and to Refs. [1-10] for global fits. Details on the framework underlying PDFs can be found in general textbooks [37-41].

\subsection{Parton distribution functions}

Quantum chromodynamics is the non-abelian quantum field theory that describes the strong interaction. It provides the theoretical foundation for the phenomenological ideas of quark model, color charge, and partons as hadron constituents. The power of QCD to describe physics from the pion mass scale all the way up to the scale of high-energy colliders, such as the LHC, relies on the remarkable properties of asymptotic freedom [42-45] and factorization [46, 47].

At high energies, or short distances, the QCD coupling is small and perturbation theory can accurately characterize the relevant scattering processes [48]. At low energies, or larger distances, nonperturbative effects give rise to quark confinement and spontaneous chiral symmetry breaking [49]. The connection between low- and high-energy dynamics is provided by QCD factorization theorems [46,47]: short-distance physics above the factorization scale $\mu$ is captured by partonic hard-scattering crosssections calculated perturbatively as a power series expansion in the QCD coupling, while the longdistance physics below the factorization scale $\mu$ is described by nonperturbative quantities. In a collinear, leading-twist factorization framework, these quantities are universal (i.e. process-independent) PDFs. Depending on the helicity state of the parent hadron, one usually distinguishes between helicity-averaged (unpolarized, henceforth) and helicity-dependent (polarized, henceforth) PDFs.

Unpolarized PDFs are denoted as

$$
f\left(x, \mu^{2}\right) \equiv f^{\rightarrow}\left(x, \mu^{2}\right)+f^{\leftarrow}\left(x, \mu^{2}\right), \quad f=\{g, u, \bar{u}, d, \bar{d}, s, \bar{s}, \ldots\},
$$

where $x$ is the fraction of the hadron longitudinal momentum carried by the parton, and the sum over parton's helicities aligned along $(\rightarrow)$ and opposite $(\leftarrow)$ the parent's nucleon helicity is made explicit. An additional index could be used to denote the hadronic species (proton, neutron, pion, ... ). However, we omit such a designation, as we only refer to the proton in this paper.

At leading order (LO) in the QCD coupling series, unpolarized PDFs describe the probability distribution of a parton with a specified momentum fraction $x$. The total momentum carried by each parton flavor is then given by the first moment of the corresponding PDF, for instance

$$
\begin{aligned}
\int_{0}^{1} d x x\left[u\left(x, \mu^{2}\right)\right] & =\langle x\rangle_{u}\left(\mu^{2}\right), \\
\int_{0}^{1} d x x\left[u\left(x, \mu^{2}\right)+\bar{u}\left(x, \mu^{2}\right)\right] & =\langle x\rangle_{u^{+}}\left(\mu^{2}\right) .
\end{aligned}
$$

Here, $\langle x\rangle_{u}$ is the momentum carried by the up-quark, and $\langle x\rangle_{u^{+}}$is the momentum carried by the sum of up and anti-up quarks ${ }^{2}$, see Appendix A for our notational conventions.

\footnotetext{
${ }^{2}$ We always refer to $q^{+}$to indicate the sum of the quark and anti-quark PDFs of the same flavor.
} 
Polarized PDFs describe the extent to which quarks and gluons with a given momentum fraction $x$ have their spins aligned with the spin direction of a fast moving nucleon in a helicity eigenstate. They are denoted as

$$
\Delta f\left(x, \mu^{2}\right) \equiv f^{\rightarrow}\left(x, \mu^{2}\right)-f^{\leftarrow}\left(x, \mu^{2}\right), \quad f=\{g, u, \bar{u}, d, \bar{d}, s, \bar{s}, \ldots\},
$$

where, as in Eq. (2.1), $x$ is the fractional momentum carried by the parton, and the parton's spin alignment along $(\rightarrow)$ or opposite $(\leftarrow)$ the polarization direction of its parent nucleon is made explicit.

Much of the interest in polarized PDFs is related to the fact that their zeroth moments can be interpreted as the fractions of the proton's spin carried by the corresponding partons. They are therefore the key to one of the most fundamental, but not yet satisfactorily answered questions in hadronic physics, i.e., how the spin of the proton is distributed among its constituents. Specifically, the zeroth moments of the singlet and the gluon polarized PDFs,

$$
\begin{aligned}
& \Delta \Sigma\left(\mu^{2}\right)=\sum_{q}^{N_{f}} \int_{0}^{1} d x\left[\Delta q\left(x, \mu^{2}\right)+\Delta \bar{q}\left(x, \mu^{2}\right)\right] \equiv \sum_{q}^{N_{f}}\langle 1\rangle_{\Delta q^{+}}\left(\mu^{2}\right), \\
& \Delta G\left(\mu^{2}\right)=\int_{0}^{1} d x \Delta g\left(x, \mu^{2}\right) \equiv\langle 1\rangle_{\Delta g}\left(\mu^{2}\right),
\end{aligned}
$$

where $N_{f}$ is the number of active flavors, directly contribute to the proton spin sum rule [50].

Beyond LO, PDFs are renormalization scheme-dependent quantities, typically worked out in the $\overline{\mathrm{MS}}$ scheme $[51,52]$. When PDFs are convolved with the appropriate partonic hard-scattering cross-sections, computed in the same scheme, the corresponding physical observables are scheme-independent, up to subleading spurious terms in the perturbative expansion.

Both unpolarized and polarized PDFs are accessible, theoretically and experimentally, through the forward Compton scattering amplitude

$$
T_{\mu \nu}(p, q, s)=\int \mathrm{d}^{4} z e^{i q z}\left\langle p, s\left|T J_{\mu}(z) J_{\nu}(0)\right| p, s\right\rangle
$$

at large virtual photon momenta $q^{2}=-Q^{2}$. Here $T$ is the time-ordering operator, $J_{\mu}(z)$ and $J_{\nu}(0)$ are vector currents at space-time points $z$ and 0 respectively, and the external states are hadronic states with momentum $p$ and spin $s$.

The most general form of the Compton amplitude $T_{\mu \nu}(p, q)$ reads [53]

$$
\begin{aligned}
T_{\mu \nu}(p, q, s)=( & \left.-g_{\mu \nu}+\frac{q_{\mu} q_{\nu}}{q^{2}}\right) \mathcal{F}_{1}\left(\omega, Q^{2}\right)+\left(p_{\mu}-\frac{p \cdot q}{q^{2}} q_{\mu}\right)\left(p_{\nu}-\frac{p \cdot q}{q^{2}} q_{\nu}\right) \frac{1}{p \cdot q} \mathcal{F}_{2}\left(\omega, Q^{2}\right) \\
& +i \epsilon_{\mu \nu \lambda \sigma} q^{\lambda} s^{\sigma} \frac{1}{p \cdot q} \mathcal{G}_{1}\left(\omega, Q^{2}\right)+i \epsilon_{\mu \nu \lambda \sigma} q^{\lambda}\left(p \cdot q s^{\sigma}-s \cdot q p^{\sigma}\right) \frac{1}{(p \cdot q)^{2}} \mathcal{G}_{2}\left(\omega, Q^{2}\right),
\end{aligned}
$$

where $\omega=2 p \cdot q / q^{2}$ and $\mathcal{F}_{1}, \mathcal{F}_{2}, \mathcal{G}_{1}$ and $\mathcal{G}_{2}$ are the Compton amplitude structure functions. They can be related to the electromagnetic structure functions $F_{1}, F_{2}, g_{1}$ and $g_{2}$, used to parametrize the deep-inelastic scattering (DIS) hadronic tensor ${ }^{3}$

$$
\begin{aligned}
W_{\mu \nu}(p, q, s)= & \frac{1}{4 \pi} \int d^{4} z e^{i q z}\left\langle p, s\left|\left[J_{\mu}(z), J_{\nu}(0)\right]\right| p, s\right\rangle \\
= & \left(-g_{\mu \nu}+\frac{q_{\mu} q_{\nu}}{q^{2}}\right) F_{1}\left(x, Q^{2}\right)+\left(p_{\mu}-\frac{p \cdot q}{q^{2}} q_{\mu}\right)\left(p_{\nu}-\frac{p \cdot q}{q^{2}} q_{\nu}\right) \frac{1}{p \cdot q} F_{2}\left(x, Q^{2}\right) \\
& +i \epsilon_{\mu \nu \lambda \sigma} q^{\lambda} s^{\sigma} \frac{1}{p \cdot q} g_{1}\left(x, Q^{2}\right)+i \epsilon_{\mu \nu \lambda \sigma} q^{\lambda}\left(p \cdot q s^{\sigma}-s \cdot q p^{\sigma}\right) \frac{1}{(p \cdot q)^{2}} g_{2}\left(x, Q^{2}\right),
\end{aligned}
$$

\footnotetext{
${ }^{3} \mathrm{~A}$ more general expression of the DIS hadronic tensor including electroweak currents can be worked out, see [54,55].
} 
where $x=1 / \omega$ is the Bjorken variable identified with the parton fractional momentum at Born level; see $[53,55]$ for details. Specifically, given the definitions in (2.8) and (2.9), the optical theorem implies that twice the imaginary part of $T_{\mu \nu}$ is equal to $W_{\mu \nu}$ times $4 \pi$. Neglecting target mass corrections, one has

$$
\begin{aligned}
& \mathcal{F}_{1}\left(\omega, Q^{2}\right)=2 \omega^{2} \int_{0}^{1} d x \frac{x F_{1}\left(x, Q^{2}\right)}{1-(\omega x)^{2}}=\sum_{n=2,4, \cdots}^{\infty} 2 \omega^{n} \int_{0}^{1} d x x^{n-1} F_{1}\left(x, Q^{2}\right) \\
& \mathcal{G}_{1}\left(\omega, Q^{2}\right)=2 \omega \int_{0}^{1} d x \frac{g_{1}\left(x, Q^{2}\right)}{1-(\omega x)^{2}}=\sum_{n=1,3, \cdots}^{\infty} 2 \omega^{n} \int_{0}^{1} d x x^{n-1} g_{1}\left(x, Q^{2}\right) .
\end{aligned}
$$

At a sufficiently high momentum transfer $Q^{2}$, power corrections can be neglected and QCD factorization allows one to write the structure functions $F_{1}\left(x, Q^{2}\right)$ and $g_{1}\left(x, Q^{2}\right)$ as a convolution between perturbatively-computable hard-scattering cross-sections and nonperturbative parton distributions:

$$
\begin{aligned}
& F_{1}\left(x, Q^{2}\right)=x \sum_{f} \int_{x}^{1} \frac{\mathrm{d} z}{z} C_{1, f}\left(\frac{x}{z}, \alpha_{s}\left(Q^{2}\right)\right) f\left(z, Q^{2}\right), \\
& g_{1}\left(x, Q^{2}\right)=\sum_{f} \int_{x}^{1} \frac{d z}{z} \Delta C_{1, f}\left(\frac{x}{z}, \alpha_{s}\left(Q^{2}\right)\right) \Delta f\left(z, Q^{2}\right) .
\end{aligned}
$$

Here, the sums run over the number of active flavors at the scale $Q^{2}$ (including the gluon), $C_{1, f}$ and $\Delta C_{1, f}$ are the perturbative partonic hard-scattering cross-sections, $\alpha_{s}$ is the QCD strong coupling, and $f\left(x, Q^{2}\right)$ and $\Delta f\left(x, Q^{2}\right)$ are the unpolarized and polarized PDFs.

Parton distributions allow for a proper field-theoretic definition as matrix elements in a hadron state of bilocal operators that act to count the number of quarks and gluons carrying a fraction $x$ of the hadron's momentum. The definitions are usually stated in the light-cone frame, where the hadron carries momentum $p$ with plus/minus components $p^{ \pm}=\left(p^{0} \pm p^{3}\right) / \sqrt{2}$, and transverse components equal to zero. For example, in the case of unpolarized and polarized quark PDFs, one has

$$
\begin{aligned}
q(x) & =\frac{1}{4 \pi} \int d y^{-} e^{-i y^{-} x p^{+}}\left\langle p\left|\bar{\psi}\left(0, y^{-}, \mathbf{0}_{\perp}\right) \gamma^{+} \mathcal{G} \psi(0,0, \mathbf{0})\right| p\right\rangle \\
\Delta q(x) & =\frac{1}{4 \pi} \int d y^{-} e^{-i y^{-} x p^{+}}\left\langle p, s\left|\bar{\psi}\left(0, y^{-}, \mathbf{0}_{\perp}\right) \gamma^{+} \gamma^{5} \mathcal{G} \psi(0,0, \mathbf{0})\right| p, s\right\rangle
\end{aligned}
$$

where $\psi$ is the quark field and $\mathcal{G}$ is an appropriate gauge link required to make Eqs. (2.14)-(2.15) gauge invariant. See Refs. [47,56-58] for the definition of $\mathcal{G}$ and for explicit light-cone formulæ of unpolarized an polarized gluon PDFs.

While PDFs cannot be calculated perturbatively, their dependence on the scale $\mu$ resulting from factorization can be. This is done by means of the DGLAP (Dokshitzer-Gribov-Lipatov-Altarelli-Parisi) evolution equations [59-61], a set of integro-differential coupled equations of the form

$$
\begin{gathered}
\frac{\partial f^{\prime}\left(x, \mu^{2}\right)}{\partial \ln \mu^{2}}=\sum_{f=g, q, \bar{q}} \int_{x}^{1} \frac{\mathrm{d} z}{z} P_{f^{\prime} f}\left(\frac{x}{z}, \alpha_{s}\left(\mu^{2}\right)\right) f\left(z, \mu^{2}\right) \\
\frac{\partial \Delta f^{\prime}\left(x, \mu^{2}\right)}{\partial \ln \mu^{2}}=\sum_{f=g, q, \bar{q}} \int_{x}^{1} \frac{\mathrm{d} z}{z} \Delta P_{f^{\prime} f}\left(\frac{x}{z}, \alpha_{s}\left(\mu^{2}\right)\right) \Delta f\left(z, \mu^{2}\right) .
\end{gathered}
$$

In short, the logarithmic derivative of the PDF is determined by a convolution of the PDFs with the unpolarized (polarized) DGLAP kernels $P_{f^{\prime} f}\left(\Delta P_{f^{\prime} f}\right)$, which can be computed perturbatively in powers 
of $\alpha_{s}$. The unpolarized splitting functions $P_{f^{\prime} f}$ are currently completely known up to NNLO [62,63] in the $\overline{\mathrm{MS}}$ renormalization scheme. Results for the unpolarized nonsinglet splitting functions have appeared recently at $\mathrm{N}^{3} \mathrm{LO}[64,65]$. The polarized splitting functions $\Delta P_{f^{\prime} f}$ are currently known up to NNLO [66] in the $\overline{\mathrm{MS}}$ scheme. The DGLAP evolution equations can be solved numerically using either $x$-space or Mellin $N$-space techniques that are widely available in various public codes [67-71]. The typical level of agreement for the results of the PDF evolution has been demonstrated to be of $\mathcal{O}\left(10^{-5}\right)[72,73]$

\section{$2.2 \quad$ Lattice QCD}

The lattice-QCD method is based on regularizing QCD on a finite Euclidean lattice and is generally studied by numerical computation of QCD correlation functions in the path-integral formalism [29, 7476], using methods adapted from statistical mechanics [77,78]. To make contact with experimental data, the numerical results are extrapolated to the continuum and infinite-volume limits. The past decade has seen significant progress in the development of efficient algorithms for the generation of ensembles of gauge field configurations and tools for extracting the relevant information from latticeQCD correlation functions. In this respect, lattice-QCD calculations have reached a level where they not only complement, but also guide current and forthcoming experimental programs [79, 80].

In this section, we discuss the sources of systematic uncertainties that affect current lattice QCD calculations, and we present lattice-QCD methods to determine either the Mellin moments of PDFs or the complete PDF $x$-dependence.

\subsubsection{Systematic uncertainties}

Lattice-QCD calculations must demonstrate control over all sources of systematic uncertainty introduced by the discretization of QCD on the lattice to make meaningful contact with experimental data. These include discretization effects that vanish in the continuum limit; extrapolation from unphysically heavy pion masses; finite volume effects; and renormalization of composite operators. To take the continuum limit requires accurate determinations of the lattice spacing. We briefly review these main sources of systematic uncertainty here; for a fuller account see, for example, Ref. [81].

- Discretization effects and the continuum limit. There is a fair degree of flexibility in discretizing the QCD action. This has led to a variety of formulations, which differ mainly in the choice of the action for quarks. In the continuum limit, which corresponds to taking the lattice spacing $a$ to zero with all physical quantities fixed, the simplest discretizations differ from continuum QCD at $\mathcal{O}(a)$. In practice, one cannot afford to perform numerical simulations at arbitrarily small lattice spacings, because the cost of computation increases with a large inverse power of the lattice spacing, and $\mathcal{O}(a)$ effects can be significant even with current lattice spacings ranging from $0.15 \mathrm{fm}$ to $0.05 \mathrm{fm}$. To accelerate the convergence to the continuum limit, improved quark and gluon actions are widely used, which include higher-dimension operators to reduce the discretization errors to $\mathcal{O}\left(a^{2}\right)$ or better. Chiral fermions with automatic $\mathcal{O}(a)$ improvement and small $\mathcal{O}\left(a^{2}\right)$ discretization errors are also adopted to admit calculations on coarser lattice spacings $[82-84]$.

- Pion mass dependence. The computational cost of the fermion contribution to the path integral increases with a large inverse power of the bare quark mass (or, equivalently, the pion mass). Lattice-QCD calculations are therefore often performed at unphysically heavy pion masses, although results calculated directly with physical pion masses have become increasingly common, albeit with larger errors. To obtain results at the physical pion mass, lattice data are generated at a sequence of pion masses and then extrapolated to the physical pion mass. To control the associated 
systematic uncertainties, these extrapolations are guided by effective theories. In particular, the pion-mass dependence can be parametrized using chiral perturbation theory $(\chi \mathrm{PT})[85]$, which accounts for the Nambu-Goldstone nature of the lowest excitations that occur in the presence of light quarks.

- Finite volume effects. Numerical lattice-QCD calculations are necessarily restricted to a finite space-time volume, e.g., a hypercube of side $L$. For most simple quantities, these effects decay exponentially with the size of the lattice $[86,87]$, and therefore the easiest way to minimize or eliminate finite volume effects is to choose the volume sufficiently large in physical units. Unfortunately, this can be prohibitively expensive as one approaches the continuum limit, requiring the number of lattice sites to grow as $L / a$ in all four directions. Finite volume $\chi \mathrm{PT}$ is the preferred tool to develop systematic expansions that provide quantitative information on finite-volume effects. In general, finite volume effects of hadrons are dominated by their interactions with pions, which can travel around the (periodic) lattice many times. Numerical evidence suggests that lattice sizes of $m_{\pi} L \geq 4$, where $m_{\pi}$ is the pion mass, are generally sufficiently large that finite volume effects are negligible for mesons, within the current precision of lattice-QCD calculations. From the studies of the pseudoscalar and electromagnetic form factors of the nucleon, it is evident that larger physical volumes are needed for the baryons.

- Excited state contamination. At small Euclidean times, a lattice-QCD correlation function is a sum over a tower of states that behave as $e^{-m_{i} t}$, where $m_{i}$ is the energy of the state and $t$ is the Euclidean time. Thus, at large Euclidean times, ground-state quantities can be extracted by fitting to the dominant exponential behavior. Unfortunately, the signal-to-noise ratio is exponentially suppressed as $e^{-\left(E_{N}-3 m_{\pi} / 2\right) t}$, where $E_{N}$ is the nucleon energy [88]. Thus, lattice-QCD results are extracted from an intermediate region in which excited state contributions are either small or well-controlled and the signal-to-noise ratio is sufficiently large that the signal can be reliably extracted. This is a particular challenge for baryons and is one of the largest sources of systematic uncertainties for nucleon matrix elements.

- Renormalization. The matrix elements extracted from a lattice-QCD calculation at a given lattice spacing are bare matrix elements, rendered finite by the presence of the lattice spacing, which serves as a gauge-invariant UV regulator. To take the continuum limit, i.e., remove the regulator, one must renormalize the corresponding operators and fields and match them to some common scheme and scale used by phenomenologists. Although renormalization is traditionally discussed in the framework of perturbation theory, at hadronic energy scales the renormalization constants should be computed nonperturbatively to avoid uncontrolled uncertainties due to truncated perturbative results. To compare with phenomenology, which uses the $\overline{\mathrm{MS}}$ scheme, a conversion factor from the nonperturbative scheme must be computed perturbatively. This requires a renormalization condition that can be implemented on the lattice and in continuum perturbation theory. In QCD with only light quarks it is technically advantageous to employ so-called mass-independent renormalization schemes. A common choice is the regularizationindependent/momentum (RI/MOM) scheme [89].

In addition, on a hypercubic lattice, the orthogonal group $O(4)$ of continuum Euclidean space-time is reduced to the hypercubic group $H(4)$. Thus, operators are classified according to irreducible representations of $H(4)$ [90]. Different irreducible representations belonging to the same $O(4)$ multiplet will, in general, give different answers at finite lattice spacing, an effect that can be reduced by improving the operators [91]. Conversely, operators that lie in different irreducible representations of $O(4)$, but the same irreducible representations of $H(4)$, will mix at finite lattice spacing but not in the continuum. When these operators have lower mass dimensions, the mixing coefficients scale with the inverse lattice spacing to some power, and diverge in the continuum limit. 
This power-divergent mixing must be removed nonperturbatively, and is a particular challenge for lattice calculations of the Mellin moments of PDFs (see Sec. 2.2.2).

- Lattice-spacing determination. Numerical lattice-QCD calculations naturally determine all dimensionful quantities in units of the lattice spacing. Thus, extracting physical values requires the determination of the lattice scale. This is achieved by matching a quantity with mass dimension to its experimental value or through a well-defined theoretical procedure, that is referred to as scale-setting. Popular reference scales include light decay constants, hadron masses, scales defined in terms of the heavy quark potential or, most recently, the length scales $\sqrt{t_{0}}[92]$ and $w_{0}$ [93] defined via the Wilson gradient flow [92]. These scales can be computed cheaply and can be used to match scales between different gauge ensembles very accurately. However, a hadron mass or a decay constant — which are known accurately from experiment and can be computed precisely in lattice-QCD — have to be used for absolute scale setting. A popular hadronic mass for this purpose is the mass of the triply strange $\Omega$ baryon [94] or the 2S-1S splitting in the Upsilon spectrum [95].

These sources of systematic uncertainty all need to be under control when confronting experimental data with lattice results, or vice versa. For a coherent assessment of the present state of lattice-QCD calculations of various quantities, the degree to which each systematic has been controlled in a given calculation is an important consideration. In Sec. 3.1.2, we characterize the quality of the lattice calculations, based on criteria inspired by the FLAG analysis of flavor physics on the lattice [81].

\subsubsection{Mellin moments of PDFs from lattice QCD}

Parton distributions cannot be directly determined in Euclidean lattice QCD, because their fieldtheoretic definition involves fields at light-like separations. Instead, the traditional approach for latticeQCD calculations has been to determine the matrix elements of local twist-two operators, where twist is the dimension minus the spin, that can be related to the Mellin moments of PDFs. In principle, given a sufficient number of Mellin moments, PDFs can be reconstructed from the inverse Mellin transform. In practice, however, the calculation is limited to the lowest three moments, because power-divergent mixing occurs between twist-two operators on the lattice. Three moments are insufficient to fully reconstruct the momentum dependence of the PDFs without significant model dependence [96]. The lowest three moments do provide, however, useful information, both as benchmarks of lattice-QCD calculations and as constraints in global extractions of PDFs. Here we briefly review the determination of Mellin moments of PDFs from lattice QCD.

Using the operator product expansion (OPE) [97], the Mellin moments of the structure functions and the corresponding PDFs can be expressed, up to higher-twist effects, in terms of matrix elements of local operators:

$$
\begin{aligned}
2 \int_{0}^{1} d x x^{n-1} F_{1}\left(x, Q^{2}\right) & =\left.\sum_{a} C_{1, a}^{n}\left(\mu^{2}\right) v_{a}^{n}\left(\mu^{2}\right)\right|_{\mu^{2}=Q^{2}}=\sum_{a} C_{1, a}^{n}\left(Q^{2}\right) \int_{0}^{1} d x x^{n-1} f_{a}\left(x, Q^{2}\right), \\
4 \int_{0}^{1} d x x^{n} g_{1}\left(x, Q^{2}\right) & =\left.\sum_{a} \Delta C_{1, a}^{n}\left(\mu^{2}\right) a_{a}^{n}\left(\mu^{2}\right)\right|_{\mu^{2}=Q^{2}}=\sum_{a} \Delta C_{1, a}^{n}\left(Q^{2}\right) \int_{0}^{1} d x x^{n} 2 \Delta f_{a}\left(x, Q^{2}\right),
\end{aligned}
$$

where $v_{i}^{n}\left(\mu^{2}\right)$ and $a_{i}^{n}\left(\mu^{2}\right)$ are reduced matrix elements of the appropriate twist-two operators [98],

$$
\begin{aligned}
\frac{1}{2} \sum_{s}\left\langle p, s\left|\mathcal{O}_{\left\{\mu_{1}, \cdots, \mu_{n}\right\}}^{i}\right| p, s\right\rangle & =2 v_{i}^{n}\left[p_{\mu_{1}} \cdots p_{\mu_{n}}-\text { traces }\right] \\
\left\langle p, s\left|\mathcal{O}_{\left\{\sigma \mu_{1}, \cdots, \mu_{n}\right\}}^{5 i}\right| p, s\right\rangle & =\frac{1}{n+1} a_{i}^{n}\left[s_{\sigma} p_{\mu_{1}} \cdots p_{\mu_{n}}-\text { traces }\right]
\end{aligned}
$$




\begin{tabular}{ccc} 
Matrix element & Operator & PDF moment \\
\hline$v_{q}^{2}, v_{\bar{q}}^{2}$ & $(\mathrm{i} / 2) \bar{q}(x) \gamma_{\mu_{1}} \overleftrightarrow{D}_{\mu_{2}} q(x)$ & $\langle x\rangle_{q^{+}}$ \\
$v_{q}^{3}, v_{\bar{q}}^{3}$ & $(\mathrm{i} / 2)^{2} \bar{q}(x) \gamma_{\mu_{1}} \overleftrightarrow{D}_{\mu_{2}} \overleftrightarrow{D}_{\mu_{3}} q(x)$ & $\left\langle x^{2}\right\rangle_{q^{-}}$ \\
$a_{q}^{0}$ & $\bar{q}(x) \gamma_{\sigma} \gamma_{5} q(x)$ & $2\langle 1\rangle_{\Delta q^{+}}$ \\
$a_{q}^{1}$ & $(\mathrm{i} / 2) \bar{q}(x) \gamma_{\sigma} \gamma_{5} \overleftrightarrow{D}_{\mu_{1}} q(x)$ & $2\langle x\rangle_{\Delta q^{-}}$ \\
$v_{g}^{2}$ & $-\operatorname{Tr} F_{\mu_{1} \alpha} F_{\mu_{2} \alpha}$ & $\langle x\rangle_{g}$ \\
\hline
\end{tabular}

Table 2.1: List of operators relevant for the computation of the lowest two Mellin moments of polarized and unpolarized PDFs. Here we indicate, for each operator, the corresponding matrix element and the specific PDF moment that can be evaluated (see Appendix A for the notation used).

and $C_{1, i}^{n}\left(\mu^{2}\right)$ and $\Delta C_{1, i}^{n}\left(\mu^{2}\right)$ are the Mellin moments of the corresponding Wilson coefficients

$$
C_{1, i}^{n}\left(\mu^{2}\right)=\int_{0}^{1} d y y^{n-1} C_{1, i}\left(y, \mu^{2}\right), \quad \Delta C_{1, i}^{n}\left(\mu^{2}\right)=\int_{0}^{1} d y y^{n} \Delta C_{1, i}\left(y, \mu^{2}\right) .
$$

The trace terms include operators with at least one factor of the metric tensor $g^{\mu_{i} \mu_{j}}$ multiplied by operators of dimension $(n+2)$ with $n-2$ Lorentz indices. The operators relevant for the lowest two moments are listed in Table 2.1. The operator $\mathcal{O}_{\mu_{1} \mu_{2}}^{q}$ decomposes into two different representations of $H(4)$ [90], each with different lattice artifacts and renormalization factors. In the continuum limit, however, both operators should lead to the same result. In contrast, the operator $\mathcal{O}_{\mu_{1} \mu_{2} \mu_{3}}^{q}$ splits into several representations of $O(4)$ transforming identically under $H(4)$ and causing the corresponding operators to mix under renormalization on the lattice.

Higher-twist contributions. The discussion so far has focused on the limit in which higher-twist contributions, suppressed by powers of the momentum transfer, have been ignored. In fact, highertwist contributions to the lowest moment of the structure function $F_{1}\left(x, Q^{2}\right)$ are found to be of $\mathcal{O}\left(1 \mathrm{GeV}^{2} / Q^{2}\right)[99]$. For lattice QCD, typically $Q^{2} \simeq 1 / a^{2}$, and at present lattice spacings this corresponds to $Q^{2}=\mathcal{O}\left(10 \mathrm{GeV}^{2}\right)$ or a higher-twist contribution of $5-10 \%$. With contributions of higher-twist included, the OPE for $F_{1}$ reads

$$
2 \int_{0}^{1} d x x F_{1}^{q}\left(x, Q^{2}\right)=\left.C_{1, q}^{2}\left(\mu^{2}\right) v_{q}^{2}\left(\mu^{2}\right)\right|_{\mu^{2}=Q^{2}}+\left.\frac{\bar{C}_{1, q}^{2}\left(\mu^{2}\right)}{Q^{2}} \bar{v}_{q}^{2}\left(\mu^{2}\right)\right|_{\mu^{2}=Q^{2}}+\cdots,
$$

where $\bar{C}_{1, q}^{2}$ and $\bar{v}_{q}^{2}\left(\mu^{2}\right)$ are the Wilson coefficient and reduced matrix element of a generic twist-four operator. Both twist-two and four contributions mix under renormalization, to the extent that the perturbative series for the Wilson coefficients $C_{1, q}^{2}\left(\mu^{2}\right)$ diverges due to the presence of infrared (IR) renormalon singularities. This ambiguity is canceled by that in the twist-four matrix element $\bar{v}_{q}^{2}\left(\mu^{2}\right)$ that arises as a result of an ultraviolet (UV) renormalon singularity [100]. If mixing effects are ignored, the uncertainties will be, at least, comparable to the power corrections themselves. Power corrections can be assessed most efficiently, and the twist expansion tested, by a direct lattice-QCD evaluation of the Compton amplitude, which we discuss in Sec. 2.2.3.

Beyond the first three moments. Moving beyond the lowest three moments requires overcoming the challenge of power-divergent mixing for lattice-QCD twist-two operators. One novel approach to this 
problem [101] builds upon the physical intuition that as long as the scale associated with the operator (for the twist-two operators, this is the renormalization scale $\mu$ ) is taken to be much larger than the hadronic scale but much smaller than the inverse lattice spacing, no singularity necessarily arises as one takes the continuum limit. The operator can still probe the correct hadron structure at the scale $\mu$, but should be insensitive to the details of the discretization of the operator at shorter distances. A simple way to incorporate an intrinsic smearing scale for an operator is to sum over bilinears of quark fields that are displaced over many lattice sites in a small (compared to the scale $1 / \mu$ ) region of Euclidean space-time (an alternative approach appears in Ref. [102]).

To ensure that the correct $S O(4)$ transformation properties of the matrix elements are recovered in the continuum limit, one must project the sum using hyper-spherical harmonics. The properties of these operators, such as their mixing patterns and scaling properties, are discussed in detail in Ref. [101]. In particular, while the classical mixing with lower and higher spin operators are both suppressed by $\sim a^{2}$ for spatially improved operators, the mixing at one-loop in lattice perturbation theory is suppressed by $\mathcal{O}\left(\alpha_{s} a\right)$ or $\mathcal{O}\left(\alpha_{s} a^{2}\right)$. The suppression depends on the lattice action used, provided that the gauge action adopted to construct the gauge-invariant bilinears is tadpole-improved and smeared over a region whose physical size is held fixed as the continuum limit is taken. In principle, this allows higher moments of PDFs to be obtained from lattice QCD, without power divergences. Numerical investigations of this approach, which requires gauge configurations with very fine lattice spacings, are underway. Other approaches that avoid power-divergent mixing have also been suggested, including coupling fictitious heavy quarks to light-quark currents [103], and calculating current correlators in position space [104]. The practical application of these ideas is yet to be studied nonperturbatively.

\subsubsection{The $x$-dependence of PDFs from lattice QCD}

While the lowest three moments of PDFs can provide important benchmarks for lattice-QCD calculations of nucleon structure, and useful constraints in global extractions of PDFs, they are not in themselves sufficient to determine the $x$-dependence of PDFs. In the following section we summarize recent approaches to determining the $x$-dependence of PDFs directly from lattice QCD.

Hadronic tensor. In principle, PDFs can be determined from hadronic tensors provided the highertwist contributions, which have different $Q^{2}$ dependence than the leading-twist, can be subtracted. Calculating the hadronic tensor in the Euclidean path-integral approach has the advantage that no renormalization is required if conserved vector currents are used in the current-current correlation and only finite renormalizations are needed for the local currents. Furthermore, since the structure functions are frame-independent, they can be calculated in any momentum frame of the nucleon. One can choose the nucleon momenta and momentum transfers judiciously to have a desirable coverage of $x$ for a given $Q^{2}$. However, the inverse Laplace transform that is needed to convert the hadronic tensor from Euclidean space to Minkowski space can be a challenge [105, 106]. Three numerical approaches, the Backus-Gilbert method [107], improved maximum entropy, and fitting with model spectral functions, are suggested to tackle this inverse Laplace-transform problem [108]. In Ref. [105] sea partons are separated into connected sea and disconnected sea contributions, based on the distinct topologies of the diagrams in a lattice computation. This distinction can help identify the impact on PDF uncertainties of improving the uncertainties associated with disconnected diagrams determined using lattice-QCD. The extended evolution equations to accommodate both the connected sea (CS) and disconnected sea (DS) partons are derived in Ref. [109].

The inversion method. The Compton amplitude $T_{\mu \nu}(p, q)$, Eq. (2.7), can be directly obtained in lattice QCD, including disconnected contributions, by a simple extension [110] of existing implementations of the Feynman-Hellmann technique to lattice QCD [111-113]. Provided one works at sufficiently 
large $Q^{2}$, the Compton amplitude will be dominated by twist-two contributions. Varying $Q^{2}$ allows one to test the twist expansion and, in particular, isolate twist-four contributions. Moreover, one can distinguish between contributions from up, down and strange quarks, connected and disconnected, by appropriate insertions of the electromagnetic current.

To compute the Compton amplitude from the Feynman-Hellmann relation, a perturbation to the QCD Lagrangian is introduced, for example,

$$
\mathcal{L}(x) \rightarrow \mathcal{L}(x)+\lambda \mathcal{J}_{3}(x), \quad \mathcal{J}_{3}(x)=Z_{V} \cos (\vec{q} \cdot \vec{x}) e_{q} \bar{q}(x) \gamma_{3} q(x)
$$

where $q$ is the quark field to which the photon is attached, and $e_{q}$ its electric charge. For simplicity, we consider the local vector current only, so that the renormalization factor $Z_{V}$ is known and no further renormalization is needed. Taking the second derivative of the nucleon two-point function

$$
\langle N(\vec{p}, t) \bar{N}(\vec{p}, 0)\rangle_{\lambda} \simeq C_{\lambda} e^{-E_{\lambda}(p, q) t}
$$

with respect to $\lambda$ on both sides, gives

$$
-\left.2 E_{\lambda}(p, q) \frac{\partial^{2}}{\partial \lambda^{2}} E_{\lambda}(p, q)\right|_{\lambda=0}=T_{33}(p, q)
$$

For $p_{3}=q_{3}=q_{4}=0$ this leaves us with

$$
T_{33}(p, q)=4 \omega^{2} \int_{0}^{1} d x \frac{x F_{1}\left(x, Q^{2}\right)}{1-(\omega x)^{2}} .
$$

Extracting the polarized structure functions requires insertions of two different currents with $\mu \neq \nu$. The idea is then to solve Eq. (2.27) for $F_{1}\left(x, Q^{2}\right)$ numerically. In Refs. [110,114] it was shown that the unpolarized structure function $F_{1}\left(x, Q^{2}\right)$ can be computed from a lattice calculation of the Compton amplitude, devoid of any renormalization and mixing issues. With the same method, PDFs can be computed directly without the need to go through the structure functions, provided $Q^{2}$ is sufficiently large that power corrections can be neglected.

Quasi-PDFs. Quasi-PDFs provide an alternative approach to determining the $x$-dependence of PDFs directly from lattice QCD $[115,116]$. In the following discussion, we focus on the flavor-nonsinglet quasi$\mathrm{PDF}$, for which we can ignore mixing with the gluon quasi-PDF. The unpolarized quark quasi-PDF is defined as the momentum-dependent nonlocal forward matrix element

$$
\begin{aligned}
\widetilde{q}\left(x, \Lambda, p_{z}\right) & =\int \frac{d z}{2 \pi} e^{-i x z p_{z}} p_{z} h\left(z, p_{z}\right) \\
h\left(z, p_{z}\right) & =\frac{1}{4 p_{\alpha}} \sum_{s=1}^{2}\left\langle p, s\left|\bar{\psi}(z) \gamma_{\alpha} e^{i g \int_{0}^{z} A_{z}\left(z^{\prime}\right) d z^{\prime}} \psi(0)\right| p, s\right\rangle
\end{aligned}
$$

where $\Lambda$ is an UV cut-off scale, such as the inverse lattice spacing $1 / a$. The Lorentz index $\alpha$ of the matrix $\gamma_{\alpha}$ is generally chosen to be spatial, $\alpha=z$, but the alternative choice $\alpha=4$ is also possible and removes part of the leading order twist-4 contamination $[117,118]$. Because $p$ is finite, the momentum fraction $x$ can be larger than unity.

The quasi-PDF is defined for nucleon states at finite momentum and must be related to the corresponding light-front $\mathrm{PDF}^{4}$, for which the nucleon momentum is taken to infinity. In the large-momentum

\footnotetext{
${ }^{4}$ In this context the term light-front PDF is used to distinguish ordinary PDFs, Eqs. (2.1)-(2.4) from quasi-PDFs, Eq. (2.28).
} 
effective field theory (LaMET) approach, the quasi-PDF $\widetilde{q}\left(x, \Lambda, p_{z}\right)$ can be related to the $p_{z}$-independent light-front $\operatorname{PDF} q\left(x, Q^{2}\right)$ through $[115,116]$

$$
\widetilde{q}\left(x, \Lambda, p_{z}\right)=\int_{-1}^{1} \frac{d y}{|y|} Z\left(\frac{x}{y}, \frac{\mu}{p_{z}}, \frac{\Lambda}{p_{z}}\right)_{\mu^{2}=Q^{2}} q\left(y, Q^{2}\right)+\mathcal{O}\left(\frac{\Lambda_{\mathrm{QCD}}^{2}}{p_{z}^{2}}, \frac{M^{2}}{p_{z}^{2}}\right),
$$

where $\mu$ is the renormalization scale, $Z$ is a matching kernel and $M$ is the nucleon mass. Here the $\mathcal{O}\left(M^{2} / p_{z}^{2}\right)$ terms are target-mass corrections and the $\mathcal{O}\left(\Lambda_{\mathrm{QCD}}^{2} / p_{z}^{2}\right)$ terms are higher-twist effects, both of which are suppressed at large nucleon momentum. A complementary approach to LaMET views the quasi-PDF as a lattice cross-section from which the light-front PDF can be factorized [119-121]. An alternative, but related, construction is proposed in Refs. [118,122] and explored in Ref. [123].

Preliminary results from lattice calculations of quasi-PDFs have been encouraging [33-36]. However, there are a number of remaining challenges that must be overcome for an ab initio determination of the $x$-dependence of PDFs directly from lattice QCD that incorporates complete control over systematic uncertainties. Lattice calculations of quasi-PDFs are subject to the same sources of systematic uncertainty that affect all lattice calculations, see Sec. 2.2. Here we focus on systematic uncertainties that are more specific to quasi-PDFs. These are uncertainties associated with the finite nucleon momentum of the lattice calculations and to the renormalization of quasi-PDFs.

- Preliminary nonperturbative studies of the quasi-PDF used nucleon momenta in the range $p_{z}=$ $2 \pi / L$ to $10 \pi / L$, where $L$ is the physical extent of the lattice, corresponding to $p_{z}=0.5$ to $2.5 \mathrm{GeV}$ [33-36]. At such low momenta, higher-twist and target mass corrections are likely to be considerable.

Target mass corrections can be removed to all orders [35], and twist-4 contributions can be removed in principle [35,118], leaving higher-twist contamination. To reduce these remaining effects starting at $O\left(\Lambda_{\mathrm{QCD}}^{2} / p_{z}^{2}\right)$, the authors of Refs. [33,35] extrapolated to infinite nucleon momentum using the fit ansatz $a+b / p_{z}^{2}$ for each value of $x$. Although the effects of finite nucleon momentum can be mitigated, a quark-model study asserts that reducing systematic uncertainties to less than $20 \%$ at moderate values of $x$ requires significantly larger values of nucleon momentum [124], and at larger values of $x$ (roughly $x \simeq 1$ ) requires nucleon momentum as large as $p_{z}>4 \mathrm{GeV}$.

The size of the nucleon momentum is currently limited by the decreasing signal-to-noise ratio at large momenta, which requires very high statistics to extract a signal. New approaches to highmomentum nucleons are being investigated, with the most promising an approach that employs momentum smearing [125]. This method has been applied to quasi-PDFs in Refs. [36, 126], demonstrating a large improvement in the signal-to-noise ratio by reaching momenta of about $2.5 \mathrm{GeV}$.

- The leading-twist quasi-PDFs and light-front PDFs are connected through the matching (or factorization) relation, Eq. (2.29). Provided the quasi and light-front PDFs share the same IR behavior, the matching kernel can be determined in perturbation theory [117]. The one-loop matching kernel including gluon channel has been recently reported [127]. The factorization of the IR structure of quasi-PDFs into light-front PDFs and an IR-safe matching kernel was claimed to hold to all orders in Refs. [119-121]. More specifically, Refs. [119,120] claim that the factorization holds to all orders provided that UV divergences are properly renormalized. However, Ref. [128] asserted that there might be subtleties beyond leading order in perturbation theory. A distinct, but similar, issue is the IR structure of extended operators in Euclidean and Minkowski space-time. There are again subtleties in perturbation theory [129], but arguments based on general field-theoretic grounds demonstrate that the quasi-PDF extracted from an Euclidean correlation function is exactly the same matrix element as that determined from the LSZ reduction formula in Minkowski space-time [130]. 
In contrast to the IR structure, the UV structure of the quasi-PDF is quite different from the UV structure of the light-front PDF: the former has both linear and logarithmic divergences, while the latter contains only logarithmic divergences. Although there are no power-divergences in dimensional regularization, quasi-PDFs determined on the lattice are regulated by the inverse lattice spacing. In the continuum limit (for which $a \rightarrow 0$, with all physical quantities held fixed) there is a divergence, associated with the length of the Wilson line $z$, that scales as $z / a$. This divergence must be removed nonperturbatively.

For a general nonlocal bilinear operator with Lorentz structure $\Gamma$, the renormalized operator $O_{\Gamma}^{(\mathrm{ren})}(z, \mu)$ is related to its bare operator $O_{\Gamma}^{(0)}(z)$ by $[131-135]$

$$
O_{\Gamma}^{(\mathrm{ren})}(z, \mu)=e^{\delta m(\mu)|z|} Z_{\psi, z}(\mu, z) O_{\Gamma}^{(0)}(z)
$$

where $\delta m$ is the mass renormalization of a test particle moving along the Wilson line of length $z$ and $Z_{\psi, z}(\mu, z)$ removes the remaining logarithmic divergences associated with the Wilson line endpoints (the quark fields). This result holds to all orders in perturbation theory: the exponentiated counterterm $\delta m(\mu)$ completely removes the linear divergence and the quasi-PDF can be renormalized multiplicatively $[136,137]$. The exponentiated counterterm can be determined using a static heavy quark potential, which shares the same power-law divergence as the nonlocal quark bilinear [126, 138-140]. An alternative approach for controlling the power divergence has been proposed in Ref. [141].

Once the linear divergence has been removed nonperturbatively, lattice perturbation theory can be used to renormalize the remaining logarithmic divergences in the quasi-PDF [129,139,140,142]. A delicate point regarding the renormalization is the mixing among certain subsets of these nonlocal operators. Such a mixing has been identified at one-loop in perturbation theory in Ref. [143] for a variety of fermion/gluon actions or nonperturbatively based on symmetries [144,145]. The mixing coefficients are necessary to disentangle the individual matrix elements for each quasi-PDF from lattice calculation data. Of particular interest is the case of the unpolarized quasi-PDF, which mixes with the scalar quasi-PDF if the Lorentz index of Eq. (2.28) is in the same direction as the Wilson line. In contrast, the polarized and transversity PDFs with a Lorentz index in the Wilson line direction do not exhibit any mixing (to one-loop in perturbation theory).

In addition, nonperturbative schemes, such as the RI/MOM scheme [89], can be used to renormalize matrix elements determined on the lattice. Nonperturbative schemes avoid the use of lattice perturbation theory at low energy scales (usually chosen to be $\mu=\pi / a$ ), although perturbative matching between renormalization schemes is still necessary for PDFs expressed in the $\overline{M S}$ scheme. Combining a nonperturbative renormalization scheme with a step-scaling procedure [146] significantly reduces perturbative truncation uncertainties by providing a nonperturbative method for reaching high energy scales. Nonperturbative renormalization methods for quasi-PDFs have recently been constructed and applied in Refs. [126, 144, 147].

These nonperturbative procedures also remove the mixing between the unpolarized quasi-PDF and the twist-3 scalar operator, which occurs for lattice regularization that break chiral symmetry, through the construction of a $2 \times 2$ mixing matrix. The mixing coefficients do not contain any divergences. Further details can be found in Refs. [144,147].

It was recently observed that potential problems with the power divergent mixing patterns of DIS operators may arise when lattice regularization is used [148]. Further investigations into this issue would be interesting.

Lattice calculations of the $x$-dependence of PDFs have not matured up to the point to control all these sources of systematic uncertainty. Recent progress, however, has led to preliminary results that 
are encouraging. Here we highlight these results for the $x$-dependence of the unpolarized and polarized PDFs extracted from lattice QCD.

Fig. 2.1 shows example results for the renormalized unpolarized PDFs from Ref. [144] and polarized PDF from Ref. [147]. In both cases, a nonperturbative renormalization procedure is applied to the bare matrix elements that appeared in earlier work [33-36,149]. For the unpolarized PDF, the calculation is carried out at a pion mass of $310 \mathrm{MeV}$, includes one-loop matching and target mass corrections at the renormalization scale $\mu^{2}=4 \mathrm{GeV}^{2}$, and the leading higher-twist $O\left(\Lambda_{\mathrm{QCD}}^{2} / p_{z}^{2}\right)$ contributions have been removed [35]. Multiple source-sink separations are used to take into account the effects of excited-state contamination, which become more important at large momentum. Mixing under renormalization has been estimated to be a small effect but is not yet computed explicitly. More recent work at the physical pion mass [150] uses a different operator to avoid mixing effects. The polarized PDF has the advantage that is free from mixing, and is computed in Ref. [147] with fully renormalized matrix element, at a pion mass of $375 \mathrm{MeV}$. The matching to $\overline{\mathrm{MS}}$ at $\mu^{2}=4 \mathrm{GeV}^{2}$ does not include any linearly divergent term, as the matrix element in coordinate space is renormalized. Note that in both cases, the antiquark asymmetry is compatible with zero within current uncertainties, contrary to earlier unrenormalized results $[33-35,149]$. This is mainly due to the rapid increase of the renormalization factor with Wilsonline length, which amplifies the finite-volume effect from truncating long-range correlations. Ref. [150] showed that this truncation causes unphysical oscillations in the sea-flavor asymmetry and proposed that the oscillations can be removed by either imposing a filter to reduce the weighting of long-range correlations or by taking the derivative of the matrix element in coordinate space. The effectiveness of both these two methods is demonstrated in Refs. [150,151].

Pseudo-PDFs. The general dependence of the matrix element $h\left(z, p_{z}\right)$ of Eq. (2.28) on the hadron momentum $p$ and the displacement of the quark and antiquark fields $z$ can be expressed as a function of the Lorentz invariants $\nu=z \cdot p$ (Ioffe time $[152,153]$ ) and $z^{2}$, where $z$ and $p$ are general 4-vectors. We can thus introduce

$$
\bar{h}\left(\nu, z^{2}\right) \equiv h\left(z, p_{z}\right) .
$$

The pseudo-PDF is then defined by the Fourier transform

$$
\mathcal{P}\left(x, z^{2}\right)=\int \frac{d \nu}{2 \pi} e^{-i x \nu} \bar{h}\left(\nu, z^{2}\right)
$$

which has support only in the physical range $x=[-1,1][118,122]$. As discussed in Refs. [118, 122], the pseudo-PDF is directly related to both the PDFs and the transverse-momentum-dependent PDFs (TMDs). In Ref. [122], using the temporal gamma matrix in the matrix element, a possible factorization of the TMD and PDF was conjectured which implies that the ratio

$$
\mathcal{M}\left(\nu, z^{2}\right)=\frac{\bar{h}\left(\nu, z^{2}\right)}{\bar{h}\left(0, z^{2}\right)}
$$

is directly related to the PDFs as

$$
\mathcal{M}\left(\nu, z^{2}\right)=Q\left(\nu, z^{2}\right)+\mathcal{O}\left(z^{2}\right)
$$

Here $Q\left(\nu, z^{2}\right)$ is the Ioffe time PDF $[152,153]$, which is just the Fourier transform of the PDFs,

$$
q\left(x, 1 / z^{2}\right)=\int \frac{d \nu}{2 \pi} e^{-i x \nu} Q\left(\nu, z^{2}\right) .
$$

The ratio in Eq. (2.33) has a well-defined continuum limit and requires no renormalization. The polynomial corrections in Eq. (2.34) are due to violations of the factorization conjecture, while the PDF 

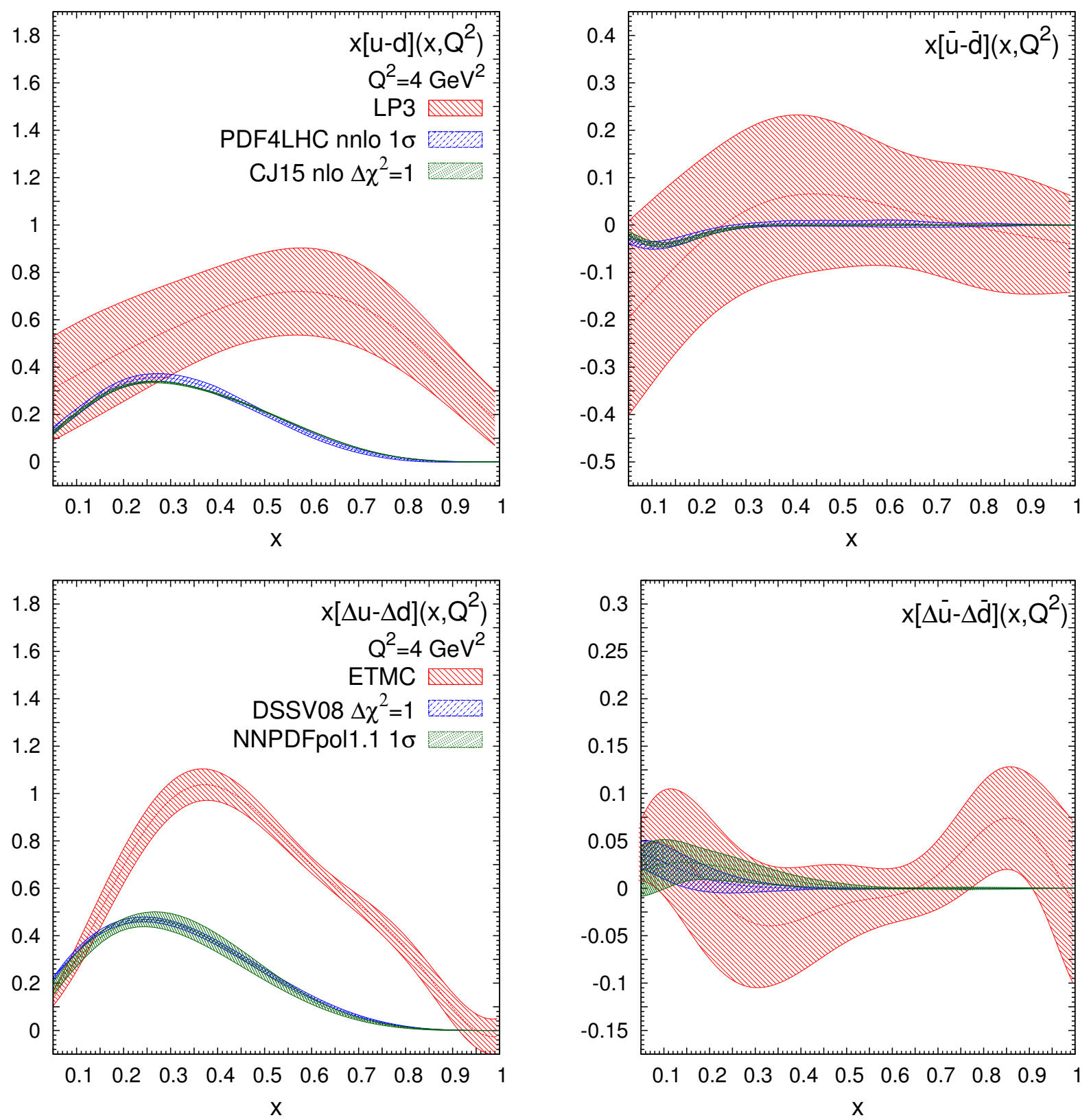

Figure 2.1: LP3's renormalized unpolarized isovector quark (top left) and antiquark (top right) PDF combinations at physical pion mass with the renormalization scale $\mu=2 \mathrm{GeV}$ [150]. ETMC's renormalized polarized isovector quark (bottom left) and antiquark (bottom right) PDF combinations at pion mass of $375 \mathrm{MeV}$ [147]. Note that only statistical errors are shown here; the systematics are yet to be addressed. The small- $x$ region $(x<0.2)$ can suffer larger systematics than the rest of the distribution due to the limited nucleon boost momentum.

$q\left(x, 1 / z^{2}\right)$ is the PDF in a particular scheme defined at scale $1 / z^{2}$. Matching to $\overline{\mathrm{MS}}$ can be performed in perturbation theory following standard methodology. One loop results can be found in Refs. [154, 155]. A preliminary study was presented in Refs. [123,156], where it was shown that indeed the conjectured factorization is observed and the residual corrections are small. Further evidence of the expected perturbative evolution of the Ioffe time PDFs was also observed. However, more detailed studies of this methodology are required. 


\subsection{Global PDF fits}

Global PDF fits realize a QCD analysis of hard-scattering measurements, often using a variety of hadronic observables. Parton distributions are parametrized at an initial energy scale, evolved up to the scale of the data via DGLAP equations (2.16)-(2.17), and used to build up the theoretical predictions for the relevant observables. In the corresponding factorization formulæ, the factorization scale, $\mu$, is usually set equal to the characteristic scale of the process, $Q$. The best-fit parameters of PDFs are then determined by minimization of a proper figure of merit, such as the log-likelihood $\chi^{2}$. In this section, we present the general global PDF fitting framework. We discuss how PDFs are determined from hard-scattering observables, paying attention to the assessment of PDF uncertainties. We highlight the theory and the data used to fit both unpolarized and polarized PDFs and present a brief review of their state-of-the-art determination.

\subsubsection{General framework}

Fitting PDFs from hard-scattering data. Parton distributions appear in the factorization formulæ of a class of sufficiently inclusive processes, among which are deep-inelastic scattering (DIS) and proton-(anti)proton collisions. The factorization formulæ for the unpolarized and polarized structure functions $F_{1}$ and $g_{1}$ were introduced in Eqs. (2.12)-(2.13). For the hadroproduction of a generic finalstate $X$ in unpolarized proton-proton $(p p)$ collisions, the corresponding factorized expression reads

$$
\sigma_{p p \rightarrow X}\left(s, \mu_{F}^{2}, \mu_{R}^{2}\right)=\sum_{a, b} \int \mathrm{d} x_{1} \mathrm{~d} x_{2} f_{a}\left(x_{1}, \mu_{F}^{2}\right) f_{b}\left(x_{2}, \mu_{F}^{2}\right) \hat{\sigma}_{a b \rightarrow X}\left(x_{1}, x_{2}, s ; \mu_{F}^{2}, \mu_{R}^{2}\right),
$$

where the unpolarized hard cross-section $\hat{\sigma}_{a b \rightarrow X}$ can be calculated perturbatively as an expansion in the QCD and electroweak (EW) running couplings. The specific values of the momentum fractions $x_{i}$ can be related to the kinematics of the final state. For example, for the production of a heavy final state, it can be shown that, at LO,

$$
x_{1,2}=\frac{M_{X}}{\sqrt{s}} e^{ \pm y_{X}}
$$

where $M_{X}$ and $y_{X}$ are the invariant mass and rapidity of the produced system and $\sqrt{s}$ is the center-ofmass energy. The factorization and renormalization scales, $\mu_{F}$ and $\mu_{R}$, are usually taken equal to the hard scale of the process, $\mu_{F}=\mu_{R}=\mu=Q$. Factorization formulæ analogous to Eq. (2.36) can be written in the polarized case for $p p$ collisions where only one or both proton beams are longitudinally polarized, see e.g. Refs. [157, 158].

When one performs a global fit, the DGLAP evolution equations of the PDFs, Eqs. (2.16)-(2.17), derive the PDFs at any scale relevant to comparisons with the data from PDF parametrizations at an arbitrary input scale, typically $Q_{0} \sim 1 \mathrm{GeV}$. The contribution of heavy quark flavors to any process are power-suppressed at scales which are below the threshold for their production [159]. Therefore, whereas in principle the QCD Lagrangian contains six quark flavors, in practice only a smaller number of active flavors $N_{f}$ are included in loops, and thus in particular in the solution of evolution equations. When expressing predictions for processes at various disparate scales in terms of a single set of PDFs it is thus necessary to use a so-called variable-flavor number (VFN) scheme, whereby different numbers of active flavors are adopted at different scales (with up to $N_{f}=5$ active flavors in most of PDF sets). Use of a fixed-flavor number (FFN) scheme only allows comparison with the data in a restricted range of scales.

The input PDF parameterization is usually chosen as

$$
f\left(x, Q_{0},\left\{a_{i}\right\}\right)=x^{a_{1}}(1-x)^{a_{2}} C\left(x,\left\{a_{j}\right\}\right),
$$

where the parameters $\left\{a_{i}\right\}$ determine the PDF shape and are different for each PDF flavor combination probed by the data. The $(1-x)^{a_{2}}$ term, with $a_{2}>0$, ensures that the PDFs vanish in the elastic limit 
$x \rightarrow 1$. Specific values of the exponent $a_{2}$ are predicted by counting rules [160], although they are not always clearly supported by phenomenological fits [161,162], and are not always used. The $x^{a_{1}}$ term governs the low- $x$ PDF behavior. It is expected from considerations based on Regge theory, which also provides the values of the exponents $a_{1}$. However, as for $a_{2}$, the value of $a_{1}$ is left free in the global fits. The interpolating function $C(x)$ in Eq. (2.38) affects the behavior of the PDFs away from the $x \rightarrow 0$ and $x \rightarrow 1$ extrapolation regions. This is assumed to be a smoothly varying function of $x$, for which a variety of parametrizations can be made.

The simplest ansatz, which has been very widely used, is to take a basic polynomial form in $x$ (or $\sqrt{x})$, such as

$$
C(x)=1+a_{2} \sqrt{x}+a_{3} x+\ldots .
$$

Functional forms of this type are, for example, taken in CJ, HERAPDF and earlier MMHT and CT sets (see below for the references to each set). More recently, the CT and MMHT collaborations expand in terms of a basis of Bernstein and Chebyshev polynomials, respectively. While formally equivalent to the simple polynomial expansion Eq. (2.39), these are much more convenient for fitting as the number of free parameters $n_{\text {par }}$ is large. In the latest CT and MMHT sets, there are between 20 and 40 free parameters in total, though some of these are kept fixed when evaluating the Hessian PDF uncertainties to reduce redundancy between the parameters. Furthermore, the use of orthogonal polynomials, like Chebyshev polynomials, allows one to decouple the parameters in $C(x)$ and to uniformly sample its possible functional shapes.

An alternative approach to the PDF parametrization Eq. (2.39) is adopted by the NNPDF collaboration. Here, the interpolating function $C(x)$ is modeled with a multi-layer feed-forward neural network (NN). In practice, this allows for a greatly increased number of free parameters, typically an order of magnitude higher than in the sets of other groups. The form of Eq. (2.38) is still assumed, but now $C(x)=\mathrm{NN}(x)$, where $\mathrm{NN}(x)$ is a neural network. The $x^{a_{1}}(1-x)^{a_{2}}$ term that multiplies $\mathrm{NN}(x)$ represents a preprocessing factor that speeds up the minimization procedure and that is determined via an iterative procedure. Because of its parametric redundancy, the neural network parametrization can be overtrained and learn the statistical noise of the data. In order to avoid such a drawback, the data are split into validation and training sets, then the best-fit is determined by cross-validation [163, 164]. A similar technique is used also in the JAM fits [18, 165].

In most of PDF sets currently in use, the PDFs for charm and heavier quarks are not parametrized as in Eq. (2.38), but rather they are generated by perturbative emission of gluons and light quarks. In the vicinity of the threshold for heavy-quark production, the quark mass cannot be neglected. It is thus necessary to explicitly include terms suppressed by powers of the heavy-quark mass in the coefficient functions, while subtracting the logarithmically enhanced, unsuppressed terms that are already generated by solving the evolution equations in order to avoid double counting. Various schemes exist so far to do so, see e.g. Refs. [5,10] for an extensive summary. The possibility of parametrizing and fitting the charm PDF on the same footing as light quark PDFs has been also explored, see e.g. [166-168] and references therein.

Once the PDF parametrization is chosen, and the theoretical details of the analysis are defined (such as the perturbative order, the treatment of heavy quarks, etc.), the best-fit PDF parameters and their uncertainty should be determined via a fitting methodology that minimizes a suitable statistical estimator, typically the $\chi^{2}$. There exist different alternative definitions of the $\chi^{2}$ to be used in the global fits $[4,14,20,169]$. For instance one frequently used definition is

$$
\chi^{2}=\sum_{i, j}^{N_{\text {dat }}}\left(T_{i}\left(\left\{a_{k}\right\}\right)-D_{i}\right)\left(\operatorname{cov}^{-1}\right)_{i j}\left(T_{j}\left(\left\{a_{k}\right\}\right)-D_{j}\right),
$$

where $N_{\text {dat }}$ is the number of data points of a given experiment, $T_{i}$ and $D_{i}$ are the corresponding theoretical predictions and experimental data, and $\left(\mathrm{cov}^{-1}\right)_{i j}$ is the inverse of the experimental covariance 
matrix. The theoretical predictions $T_{i}\left(\left\{a_{k}\right\}\right)$ depend on the input set of parameters $\left\{a_{k}\right\}$ via the PDF parametrization, see Eq. (2.38). Therefore, Eq. (2.40) assesses the agreement between theory and data.

The covariance matrix $(\mathrm{cov})_{i j}$ accounts for the various sources of experimental systematic uncertainties and also allows for several different definitions. One example is the so-called $t_{0}$ prescription [170], where a fixed theory prediction $T_{i}^{(0)}$ is used to define the contribution to the $\chi^{2}$ from the multiplicative systematic uncertainties, namely

$$
(\mathrm{cov})_{i j}=\delta_{i j} \sigma_{\text {stat }}^{2}+\sum_{\alpha=1}^{N_{c}} \sigma_{i, \alpha}^{(c)} \sigma_{j, \alpha}^{(c)} D_{i} D_{j}+\sum_{\beta=1}^{N_{\mathcal{L}}} \sigma_{i, \beta}^{(\mathcal{L})} \sigma_{j, \beta}^{(\mathcal{L})} T_{i}^{(0)} T_{j}^{(0)}
$$

Here $\sigma_{\text {stat }}$ is the uncorrelated uncertainty, and $\sigma_{i, \alpha}^{(c)}\left(\sigma_{i, \beta}^{(\mathcal{L})}\right)$ are the various sources of additive (multiplicative) systematic uncertainties. The $t_{0}$ prescription is needed to avoid the D'Agostini bias [171, 172], a downwards bias of the statistical estimators for the central value and the uncertainty of the theoretical predictions due to the rescaling induced by multiplicative uncertainties. See $[4,170]$ and references therein for details and the alternative penalty-trick prescription, and [169] for the alternative extended-t prescription.

PDF uncertainties. Determining the best-fit values of the PDF parameters is not enough: one also needs to estimate the associated PDF uncertainties, possibly separated into the various sources of experimental, methodological and theoretical uncertainties. In this respect, there are two main methods to determine PDF uncertainties, the Hessian and the Monte Carlo (MC) methods. ${ }^{5}$

The Hessian method [174] is based on the parabolic expansion of the $\chi^{2}$ in the vicinity of its minimum

$$
\Delta \chi^{2} \equiv \chi^{2}-\chi_{\min }^{2}=\sum_{i, j=1}^{n_{\mathrm{par}}} H_{i j}\left(a_{i}-a_{i}^{0}\right)\left(a_{j}-a_{j}^{0}\right)
$$

where the $n_{\text {par }}$ PDF fit parameters are denoted by $\left\{a_{1}, \ldots, a_{n_{\text {par }}}\right\}$, the best-fit values that minimize the $\chi^{2}$ are indicated by $\left\{a_{1}^{0}, \ldots, a_{n_{\mathrm{par}}}^{0}\right\}$, and the Hessian matrix is defined as

$$
\left.H_{i j} \equiv \frac{1}{2} \frac{\partial^{2} \chi^{2}}{\partial a_{i} \partial a_{j}}\right|_{\{\vec{a}\}=\left\{\vec{a}^{0}\right\}}
$$

By diagonalizing this Hessian matrix, it becomes possible to represent PDF uncertainties in terms of orthogonal eigenvectors within a fixed tolerance $T=\sqrt{\Delta \chi^{2}}$. These eigenvectors can then be used to estimate the PDF uncertainty for arbitrary cross-sections, using the master formula of Hessian PDF sets for the uncertainty of the cross-section $\mathcal{F}$, such as [175]

$$
\sigma_{\mathcal{F}}=\frac{1}{2}\left(\sum_{i, j}^{n_{\mathrm{par}}}\left[\mathcal{F}\left(S_{i}^{+}\right)-\mathcal{F}\left(S_{i}^{-}\right)\right]^{2}\right)^{1 / 2}
$$

where $S_{i}^{ \pm}$correspond to the $i$-th eigenvector associated with positive and negative variations with respect to the best fit value.

The Monte Carlo method [163,164,176,177] is based on constructing a representation of the probability distribution of the experimental data in terms of a large number $N_{\text {rep }}$ of replicas, which encode all the information on central values, variances and correlations provided by the experiments. Specifically,

\footnotetext{
${ }^{5}$ The Lagrange multiplier method [173] is also frequently used for dedicated studies of PDF uncertainties.
} 
given an experimental measurement of a hard-scattering observable $F_{I}^{(\exp )}$, with total uncorrelated uncertainty $\sigma_{I}^{\text {(stat) }}, N_{\text {sys }}$ fully correlated systematic uncertainties $\sigma_{I, c}^{\text {(corr) }}$ and $N_{a}\left(N_{r}\right)$ absolute (relative) normalization uncertainties $\sigma_{I, n}^{(\text {norm })}$, the Monte Carlo replicas are constructed using the expression

$$
F_{I}^{(\text {art })(k)}=S_{I, N}^{(k)} F_{I}^{(\exp )}\left(1+\sum_{c=1}^{N_{\mathrm{sys}}} r_{I, c}^{(k)} \sigma_{I, c}^{(\mathrm{corr})}+r_{I}^{(k)} \sigma_{I}^{(\mathrm{stat})}\right), \quad k=1, \ldots, N_{\mathrm{rep}}
$$

where $S_{I, N}^{(k)}$ is a normalization prefactor. The variables $r_{I, c}^{(k)}, r_{I}^{(k)}, r_{p, n}^{(k)}$ are univariate Gaussian random numbers. For each individual replica, the random fluctuations associated with a given fully-correlated systematic uncertainty will be the same for all data points, $r_{I, c}^{(k)}=r_{I^{\prime}, c}^{(k)}$.

Parton distribution fits are then performed separately on each of the Monte Carlo replicas. The resulting ensemble of PDFs samples the probability density in the space of PDFs. The expectation function of a generic observable $\mathcal{F}[\{f\}]$, depending on the fitted set of PDFs $\{f\}$, is evaluated as an average over the replica sample,

$$
\langle\mathcal{F}[\{f\}]\rangle=\frac{1}{N_{\text {rep }}} \sum_{k=1}^{N_{\text {rep }}} \mathcal{F}\left[\left\{f^{(k)}\right\}\right] .
$$

The corresponding uncertainty is determined as the variance of the Monte Carlo sample,

$$
\sigma_{\mathcal{F}}=\left(\frac{1}{N_{\text {rep }}-1} \sum_{k=1}^{N_{\text {rep }}}\left(\mathcal{F}\left[\left\{f^{(k)}\right\}\right]-\langle\mathcal{F}[\{f\}]\rangle\right)^{2}\right)^{1 / 2}
$$

Likewise, other properties of the underlying PDF probability distribution, such as skewness and kurtosis, could be readily computed.

Given a PDF set in the Hessian representation, it is possible to construct the corresponding Monte Carlo representation [178,179] and vice-versa [180,181].

So far, we discussed PDF uncertainties following from propagation of the uncertainty of the experimental data that underlie the PDF determination. Procedural uncertainties, associated with the methodology used to determine PDFs from data, can also be accounted for in the MC or Hessian approaches, or reduced to a negligible size, as in the NNPDF approach. There are however additional sources of uncertainty, mostly theoretical, that are not accounted for, either in the Hessian or in the MC methods. These are extensively discussed in Refs. [5, 8-10] and briefly summarized as follows.

- The uncertainty due to finite uncertainties associated with the input values of the physical parameters used in the global fit, such as $\alpha_{s}\left(m_{Z}\right)$ and the charm mass $m_{c}\left(m_{c}\right)$, is evaluated by repeating the fits for different values of the physical parameters and then by suitably combining the results.

- The missing higher order uncertainty (MHOU), due to the truncation of the perturbative expansion, is usually inferred by comparing NLO to NNLO unpolarized PDFs and LO to NLO polarized PDFs. While this is expected to be small for NNLO fits, currently its size is unknown.

- The uncertainty due to different choices in the treatment of heavy quarks was studied in Refs. [182, 183], for unpolarized PDFs, by looking at their impact. It was found that differences may not be entirely negligible at NLO in the vicinity of the quark threshold, though they rapidly decrease at NNLO [182].

- Uncertainties associated with missing higher-twist (power-suppressed) corrections (if they are not included in the factorized description of fit observables) are kept under control by removing data, 
below some low cut-off scale, that may be affected by them. Their impact can be studied by varying this cut-off $[184,185]$, or by looking at the stability of the fit with and without inclusion of higher twist terms $[14,15,18]$.

- Uncertainties associated with nuclear corrections, whenever they are not included, affect some DIS data in which targets are deuterons or heavier nuclei, rather than just protons. They have been studied by including such corrections according to various models [15, 18, 186, 187], or by attempting to fit the corrections directly [186, 188].

- Extrapolation uncertainties in the region not covered by experimental data are particularly delicate as far as full moments of PDFs are concerned. They are difficult to quantify, especially in the polarized case at small $x$ due to the lack of data. The impact of extrapolation uncertainties in the unpolarized case at large $x$ has been studied in $[9,189]$.

At present, the only way of dealing with such uncertainties is to make sure that they are small enough (in comparison to the data uncertainty) in each PDF set. Therefore, in the remainder of this paper, we will assume that they can be neglected. We will point out to the reader how global-fit results can be affected by underestimation of these uncertainties in Secs. 3.1.3-3.2.

\subsubsection{Unpolarized PDFs}

Theoretical features. While the general $x$ dependence of the PDFs is determined by nonperturbative QCD dynamics, there are still a number of theoretical constraints that any PDF set should satisfy. These should be imposed during the fit procedure.

First, since the proton has the quantum numbers of two up quarks and one down quark, the following quark number sum rules, given in terms of zeroth moments, must be satisfied:

$$
\begin{aligned}
\int_{0}^{1} d x\left[u\left(x, \mu^{2}\right)-\bar{u}\left(x, \mu^{2}\right)\right] & =\langle 1\rangle_{u^{-}}=2 \\
\int_{0}^{1} d x\left[d\left(x, \mu^{2}\right)-\bar{d}\left(x, \mu^{2}\right)\right] & =\langle 1\rangle_{d^{-}}=1 \\
\int_{0}^{1} d x\left[s\left(x, \mu^{2}\right)-\bar{s}\left(x, \mu^{2}\right)\right] & =\langle 1\rangle_{s^{-}}=0 .
\end{aligned}
$$

Similar constraints hold for heavy quarks: $\langle 1\rangle_{c^{-}}=\langle 1\rangle_{b^{-}}=\langle 1\rangle_{t^{-}}=0$. The valence sum rules, Eqs. (2.48), should be satisfied at any scale $\mu$. Indeed it can be shown that if they hold at the input parametrization scale $\mu=Q_{0}$, they are subsequently respected by DGLAP evolution. Therefore, for these distributions we must have $a_{1}>-1$ in Eq. (2.38), otherwise Eqs.(2.48) would be ill-defined.

Second, PDFs should satisfy the conservation of energy-momentum derived from the QCD Lagrangian. In other words, the proton's total momentum should be equal to the sum of the momentum carried by all its constituents (the so-called momentum sum rule):

$$
1=\langle x\rangle_{g}+\langle x\rangle_{u^{+}}+\langle x\rangle_{d^{+}}+\langle x\rangle_{s^{+}}+\langle x\rangle_{c^{+}}+\langle x\rangle_{b^{+}}+\langle x\rangle_{t^{+}}+\ldots
$$

where the ellipsis represents any other partonic components (such as a photon). The first moments, $\langle x\rangle_{f}$, are defined in analogy to Eqs. (2.2)-(2.3). In order to avoid a divergent contribution, we must have $a_{1}>-2$ in Eq. (2.38) for the non-valence distributions. Typically it turns out that $-2<a_{1}<-1$ for such distributions, hence the number of soft partons grows very quickly at small $x$, although the momentum fraction carried by them is well-defined and finite. As in the case of the valence sum rules, the momentum sum rule is preserved by the DGLAP evolution equations. 
Theoretical calculations of DIS and hadronic cross-sections at the highest perturbative order available should be used. Currently, this implies using NNLO for the QCD corrections and NLO for the EW and photon-induced effects $[190,191]$. Thanks to recent progress in higher-order calculations, these results are available for most of the processes entering the global PDF fits [23, 24, 192-194], including differential distributions with colored particles in the final state.

These calculations should be provided in a format such that the evaluation of the hadronic crosssections, Eq. (2.36), is not too burdensome from a computational point of view. To bypass the limitations of the lengthy (N)NLO computations, a number of fast interfaces have been developed that allow for the efficient calculation of NLO (and NNLO) fully differential hadronic cross-sections, among which APPLgrid [195], FastNLO [196] and aMCfast [197].

Experimental data. A broad set of input hard-scattering cross-sections from DIS and proton(anti)proton collisions, providing information on the PDFs over a wide range of $x$ and for different flavor combinations, is used in modern PDF fits. Inclusive DIS measurements have been realized with electron, muon and neutrino (and the corresponding antiparticles) off protons, deuterons and heavy nuclear targets. While traditional PDF fits were based mostly on DIS structure functions, and DrellYan and inclusive jet cross-sections, in recent years many other processes have proved important for constraining PDFs, among which top-quark pair production [25], the $p_{T}$ distribution of $Z$ bosons [23] and $D$ meson production in the forward region [22].

In Fig. 2.2 we show the representative kinematic coverage in the $\left(x, Q^{2}\right)$ plane of the DIS and proton(anti)proton hard-scattering measurements that are used as input in a typical global fit of unpolarized PDFs, in this case NNPDF3.1 [11]. In order to facilitate visualization, different datasets have been clustered together into families of related processes. For hadronic cross-sections, LO kinematics is assumed to map each experimental bin into a pair of points in the $\left(x, Q^{2}\right)$ plane. The fact that similar regions in the $\left(x, Q^{2}\right)$ plane are covered by different processes is essential to achieve quark flavor separation and to constrain the gluon PDF.

Abundant precise data from SLAC and Jefferson Lab exist also in the bottom right corner of the $\left(x, Q^{2}\right)$ plane, where however power corrections need to be accounted for in QCD fits $[14,15,198]$. They are not shown in Fig. 2.2 because they are excluded from the NNDPF3.1 fit by the kinematic cut on the invariant mass of the final state $W^{2}<12.5 \mathrm{GeV}^{2}$ adopted there.

State-of-the-art global PDF fits. Various collaborations provide regular updates of their global unpolarized PDF fits. The latest fits from the three main global fitting collaborations are CT14 [13], MMHT14 [12] and NNPDF3.1 [11]. These fits are performed up to NNLO in the strong coupling (with central value $\alpha_{s}\left(m_{Z}\right)=0.118$ ), and include data from the HERA $e^{ \pm} p$ collider, fixed (nuclear and proton) target experiments, the Tevatron $p \bar{p}$ collider and the LHC. The ABMP16 [14] set fits to a similar global data set (although excluding jet production) but differs in its treatment of errors, heavy flavors and the low- $Q^{2}$ and large- $x$ regions. The HERAPDF2.0 [20] set fits to the final combined HERA Run I + II data set only, with the aim of determining the PDFs from a completely consistent DIS data sample; in $x$ regions that are less constrained by HERA data, the uncertainties can be quite large. The CJ15 [15] NLO set focuses on constraining the PDFs at higher $x$ by lowering $Q^{2}$ and $W^{2}$ cuts in DIS. This greatly increases the amount of available data, but requires additional modeling of power-like $\mathcal{O}\left(1 / Q^{2}\right)$ corrections.

The features of each PDF set have been discussed in detail in Refs. [8,9], including the dataset, the fitting methodology, the theoretical details of the corresponding QCD analyses, and, most importantly, the uncertainties coming from each of these aspects.

In Fig. 2.3 we present a snapshot of the current understanding of the proton structure in the global PDF fitting framework. We compare the CT14, MMHT2014 and NNPDF3.1 NNLO PDF sets at $Q=100 \mathrm{GeV}$, normalized to the central value of the last. From top to bottom and from left to right we 


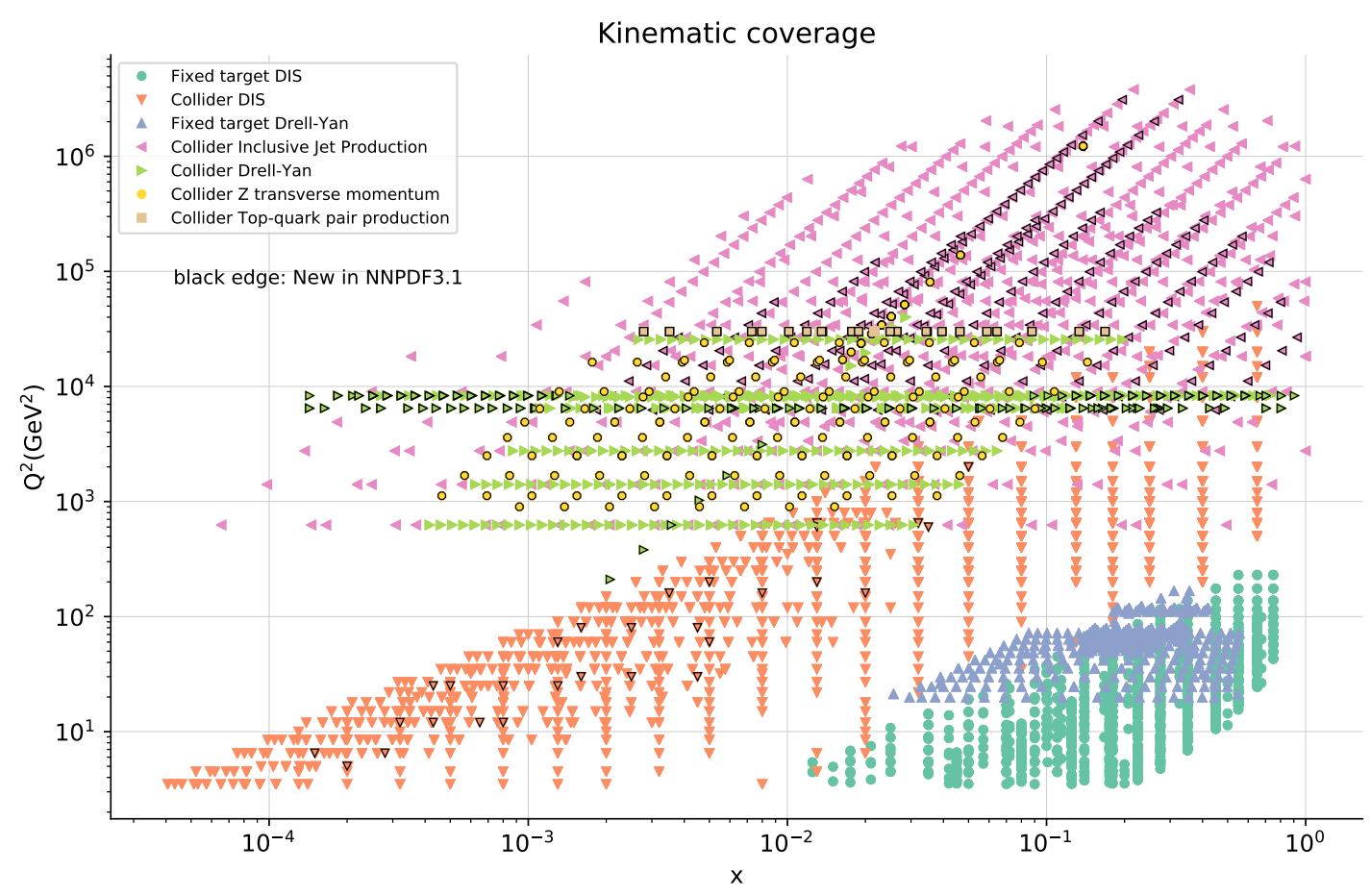

Figure 2.2: Representative kinematic coverage in the $\left(x, Q^{2}\right)$ plane of the DIS and proton-(anti)proton hardscattering measurements that are used as input in a typical fit of unpolarized PDFs, NNPDF3.1 [11]. Different datasets have been clustered together into families of related processes. For hadronic cross-sections, leading order kinematics is assumed to map each experimental bin to a pair of points in the $\left(x, Q^{2}\right)$ plane. Additional precise data from SLAC and Jefferson Lab exist also in the bottom right corner of the $\left(x, Q^{2}\right)$ plane, although they were excluded from the NNPDF3.1 fit by the cut on the invariant mass of the final state $W^{2}<12.5 \mathrm{GeV}^{2}$ adopted there.

show the $u, \bar{d}$ and $s$ quark PDFs and the gluon PDF. The error bands indicate the $68 \%$-confidence level (CL) PDF uncertainties associated with each set, computed with the corresponding master formula. We observe that differences for the up quark PDF are small, at the few percent level, but greater differences are observed for the sea quarks, in particular in the medium and large- $x$ region. For the gluon there is reasonable agreement except in the large- $x$ region, where NNPDF3.1 is softer than CT14 and MMHT14. Any other comparison plots between PDFs can be straightforwardly obtained using the APFEL-Web online plotting interface [199].

In addition to these latest versions of the global PDF fits, there has recently been a significant development of techniques aiming to construct combined PDF sets that are based on a small number of Hessian eigenvectors or MC replicas and thus are more efficient to use in lengthy higher-order computations or Monte Carlo simulations. In particular, the PDF4LHC15 PDF sets are based on the combination of the CT14, MMHT14 and NNPDF3.1 NNLO PDF sets, subsequently reduced to a small number of eigenvectors (replicas) using the META-PDF [180] and MC2H [181] (CMC [200]) compression algorithms. In this respect, Specialized Minimal PDF sets [201] (SM-PDFs) have also been advocated, which are tailored to specific physical processes and are based on a minimal number of Hessian eigenvectors.

The PDF4LHC15 NLO set [8] is displayed in Fig. 2.4 at $\mu^{2}=Q^{2}=4 \mathrm{GeV}^{2}$ and at $\mu^{2}=Q^{2}=$ $10^{2} \mathrm{GeV}^{2}$. Specifically, we show the $u_{v}=u-\bar{u}$ and $d_{v}=d-\bar{d}$ valence combinations, the $\bar{u}, \bar{d}, s$ and $c$ sea quark PDFs, and the gluon (divided by a factor 10). The evolution between $Q^{2}=4 \mathrm{GeV}^{2}$ to $Q^{2}=10^{2} \mathrm{GeV}^{2}$ is completely determined by the solution of the DGLAP evolution equations. The shape of the $u_{v}\left(u^{-}\right)$and $d_{v}\left(d^{-}\right)$valence quark combinations reflects the constraints from the valence sum 
$\mathrm{NNLO}, \mathrm{Q}=100 \mathrm{GeV}$
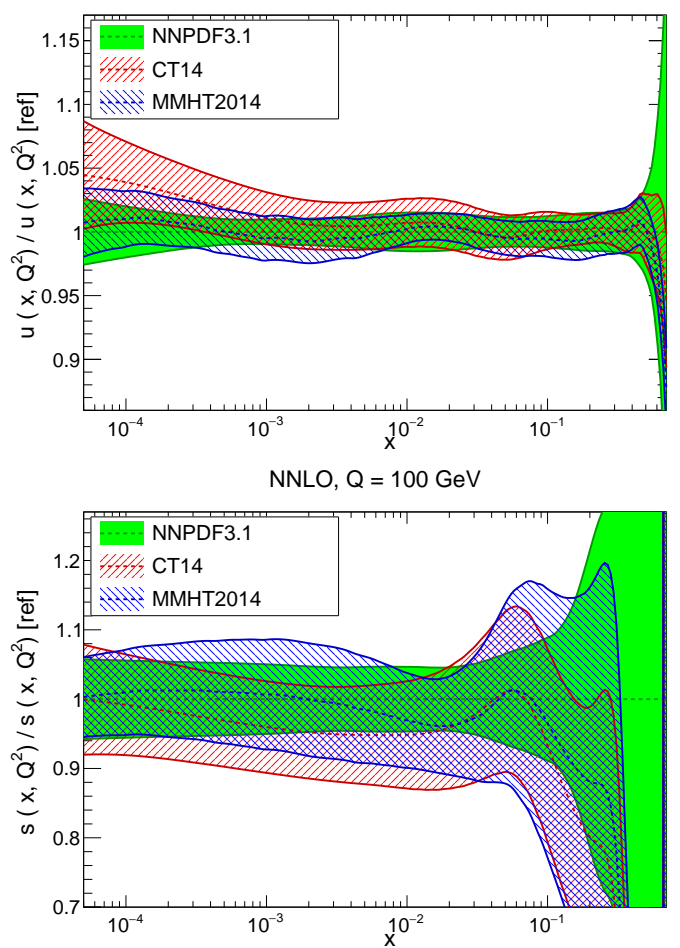

NNLO, $Q=100 \mathrm{GeV}$
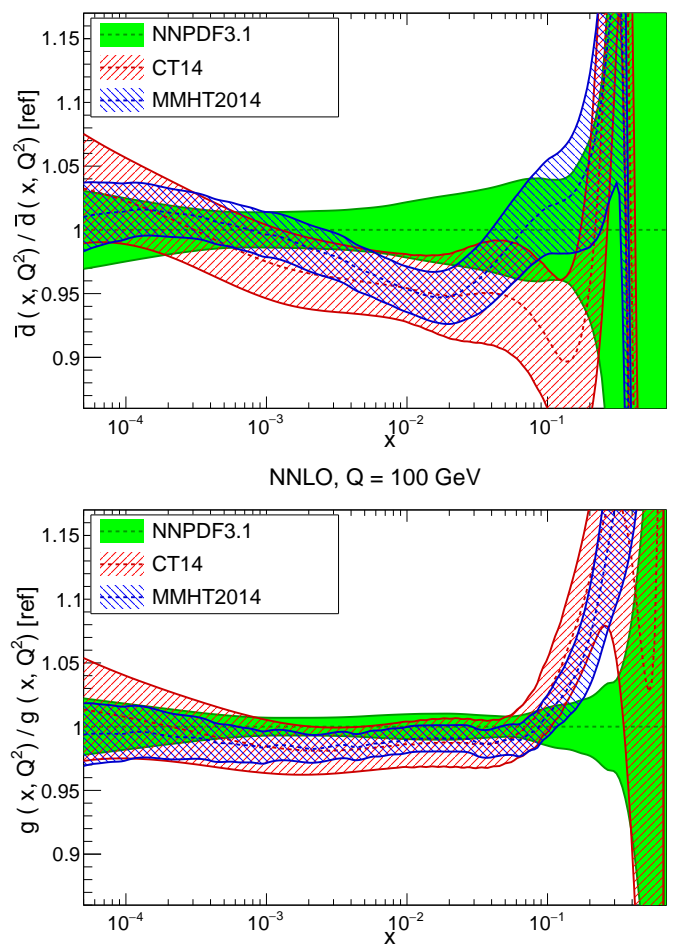

Figure 2.3: Comparison between the CT14, MMHT2014 and NNPDF3.1 NNLO PDF sets at $Q=100 \mathrm{GeV}$, normalized to the central value of the latter. From top to bottom and from left to right we show the $u, \bar{d}$ and $s$ quark PDFs as well as the gluon. The error bands indicate the 1- $\sigma$ PDF uncertainties associated with each set. These PDF comparison plots have been produced using the APFEL-Web online plotting interface [199].

rules Eq. (2.48). At small $x$, there is a rapid growth of the gluon and the sea quark PDFs, implying that the higher the collision center-of-mass energy $\sqrt{s}$, the more important gluon- and sea-quark-initiated processes become. The bands in Fig. 2.4 represent the 68\% CL PDF uncertainties.

We strongly encourage the community to use the most recent versions of global PDF fits when comparing with existing or new lattice QCD calculations. Comparing with deprecated sets, based on obsolete methodology and in many cases experimental data that have been superseded, should always be avoided.

\subsubsection{Polarized PDFs}

Theoretical features. The dependence on the momentum fraction $x$, fixed by nonperturbative QCD dynamics, should satisfy some theoretical constraints. First, PDFs must lead to positive cross-sections. At leading order (LO), this implies that polarized PDFs are bounded by their unpolarized counterparts ${ }^{6}$, $\left|\Delta f\left(x, \mu^{2}\right)\right| \leq f\left(x, \mu^{2}\right)$ [202]. Second, PDFs must be integrable: this corresponds to the assumption that the nucleon matrix element of the axial current for each flavor is finite. Third, SU(2) and SU(3) flavor symmetry, if assumed to be exact, imply that the zeroth moments of the nonsinglet $\mathcal{C}$-even PDF combinations, $\Delta T_{3}=\Delta u^{+}-\Delta d^{+}$and $\Delta T_{8}=\Delta u^{+}+\Delta d^{+}-2 \Delta s^{+}\left(\right.$where $\left.\Delta q^{+}=\Delta q+\Delta \bar{q}, q=u, d, s\right)$,

\footnotetext{
${ }^{6}$ Beyond LO, more complicated relations hold [202]; however they have little effect on PDFs.
} 

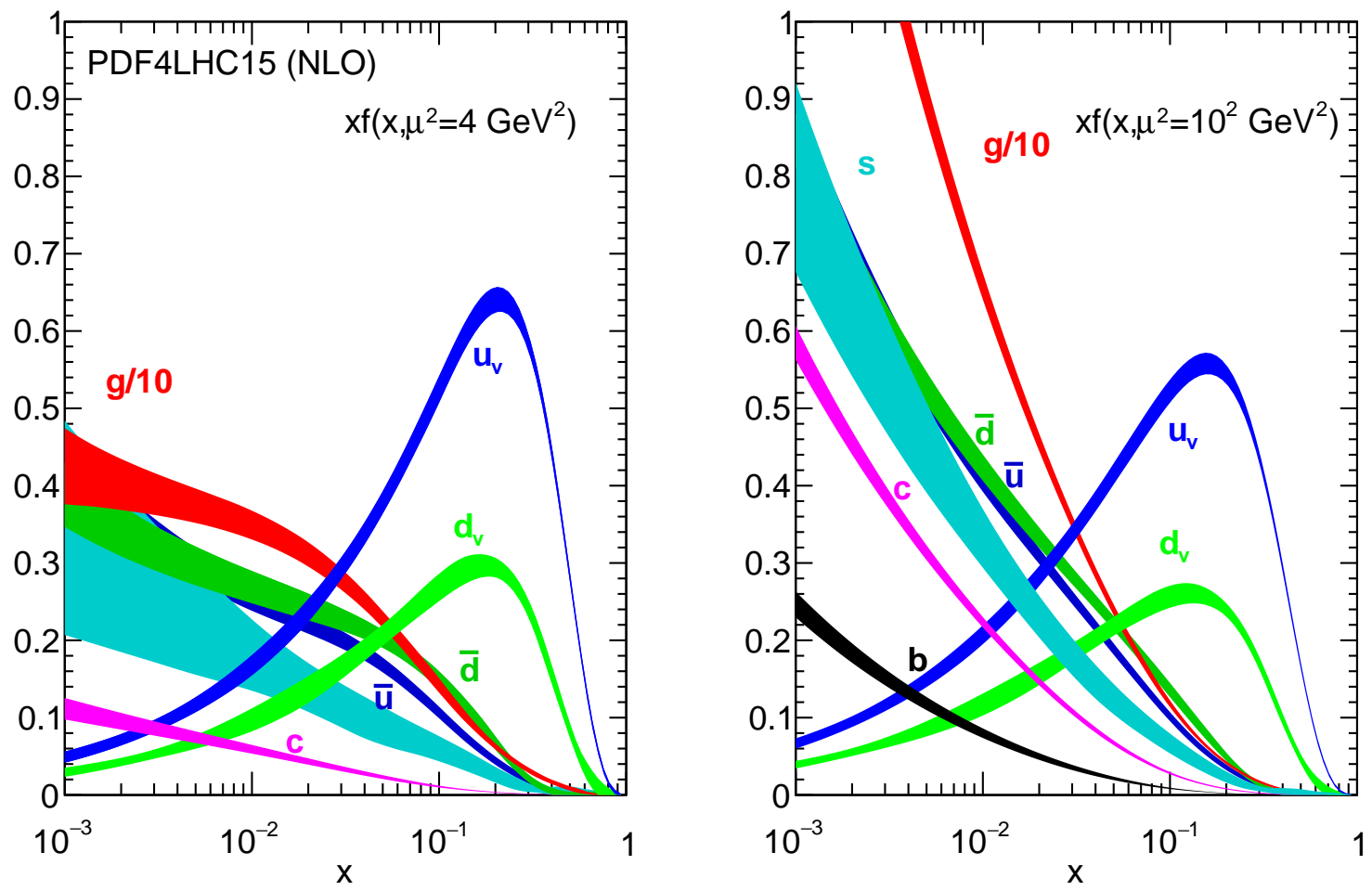

Figure 2.4: The PDF4LHC15 NLO PDFs at a low scale $\mu^{2}=Q^{2}=4 \mathrm{GeV}^{2}$ (left plot) and at $\mu^{2}=Q^{2}=$ $10^{2} \mathrm{GeV}^{2}$ (right plot) as a function of $x$. We show the $u_{v}$ and $d_{v}$ valence combinations, the $\bar{u}, \bar{d}, s$ and $c$ sea quark PDFs, and the gluon (note that the latter is divided by a factor 10).

are respectively related to the baryon octet $\beta$-decay constants, whose measured values are [28]

$$
\begin{aligned}
g_{A}=a_{3} & =\int_{0}^{1} d x \Delta T_{3}\left(x, \mu^{2}\right)=\langle 1\rangle_{\Delta u^{+}}-\langle 1\rangle_{\Delta d^{+}}=1.2723 \pm 0.0023 \\
a_{8} & =\int_{0}^{1} d x \Delta T_{8}\left(x, \mu^{2}\right)=\langle 1\rangle_{\Delta u^{+}}+\langle 1\rangle_{\Delta d^{+}}-2\langle 1\rangle_{\Delta s^{+}}=0.585 \pm 0.025 .
\end{aligned}
$$

Fairly significant violations of SU(3) symmetry are advocated in the literature (see e.g. Ref. [203] for a review). In this case, an uncertainty on the octet axial charge, which could be as large as $30 \%$ of the experimental value of $a_{8}$ in Eq. (2.51), see Ref. [204].

Experimental data. The bulk of the experimental information on polarized PDFs comes from neutral-current (photon exchange) inclusive and semi-inclusive deep-inelastic scattering (DIS and SIDIS) with charged lepton beams and nuclear targets. As photon scattering does not distinguish quarks and antiquarks, inclusive DIS data constrain only the total quark combinations $\Delta q^{+}$, while SIDIS data with identified pions or kaons in the final state constrain individual quark and antiquark flavors. In principle, both DIS and SIDIS are also sensitive to the gluon distribution $\Delta g$, as it directly enters the factorized expressions of the corresponding structure functions beyond LO, and indirectly via DGLAP evolution. In practice, the constraining power of DIS and SIDIS data on $\Delta g$ is rather weak because the $Q^{2}$ range covered by the data is limited, especially if one restricts to the kinematic region not affected by power-suppressed corrections and very precise data from JLab are therefore excluded.

Note that, in the case of SIDIS, a reliable knowledge of fragmentation functions (FFs) is required in the factorized expressions of the corresponding observables. Since FFs are nonperturbative objects 
on the same footing as PDFs, they are an additional source of uncertainty in PDF determinations, if not a bias. A significant experimental and theoretical effort has been invested in improving the independent determination of FFs [205-209] and most recently in simultaneously fitting both PDFs and FFs $[165,210]$.

Besides DIS and SIDIS fixed-target data, a significant amount of data from longitudinally polarized proton-proton collisions at the Relativistic Heavy Ion Collider (RHIC) has become available recently (see e.g. Ref. [211] for an overview), although in a limited range of momentum fractions, $0.05 \lesssim x \lesssim$ 0.4. On the one hand, longitudinal (parity-violating) single-spin and (parity-conserving) double-spin asymmetries for $W^{ \pm}$boson production are sensitive to the flavor decomposition of polarized quark and antiquark distributions, because of the chiral nature of the weak interaction [212]. On the other hand, double-spin asymmetries for jet, di-jet and $\pi^{0}$ production are directly sensitive to the gluon polarization in the proton, because of the dominance of gluon-gluon and quark-gluon initiated subprocesses in the kinematic range accessed by RHIC [213].

The kinematic coverage of the data that can be used to constrain polarized PDFs is displayed in Fig. 2.5. A comparison with Fig. 2.2 makes it apparent that the quantity of data points, their kinematic coverage and the variety of available hard-scattering processes are presently much more limited in the polarized case than in the unpolarized case. Therefore, polarized PDFs can currently be determined with much less precision than their unpolarized counterparts and only over an $x$-range limited to $x \gtrsim 0.005$. The kinematic coverage is expected to be significantly extended in the future, with DIS and SIDIS data from JLab-12 [214] and a polarized high-energy Electron-Ion Collider (EIC) [215]. Such an extended kinematic coverage is also displayed in Fig. 2.5, where it is denoted as eRHIC.

A representative illustration of polarized PDFs obtained from a global QCD analysis, namely NNDPFpol1.1 [16], is provided in Fig. 2.6. The format is the same as for the unpolarized case, Fig. 2.4, in order to ease any comparison between the two. In particular, note the suppression of all polarized PDFs at small values of $x$, including polarized sea quark PDFs, with respect to their unpolarized counterparts.

State-of-the-art global PDF fits. Several modern determinations of polarized PDFs of the proton (up to $\mathrm{NLO}^{7}$ and mostly in the $\overline{\mathrm{MS}}$ factorization scheme) are available in the literature [16-19, 27, 219-223]. A key goal of these is to unveil the size (and uncertainty) of $\Delta \Sigma$ and $\Delta G$ in Eq. (2.6). The various determinations differ among each other in the data sets included in the analysis, in some details of the QCD analysis (like the treatment of higher-twist corrections) and in the procedure used to determine PDFs from the data (for details, see e.g. Chap. 3 in Refs. [224] and [6,219]). The NNDPF procedure and the standard (adopted by DSSV) have already been outlined in Sec. 2.3.1. We note that DSSV has developed a method based on Mellin moments of the PDFs in order to efficiently incorporate NLO computations of proton-proton cross-sections in the fitting procedure. The JAM collaboration has implemented a new approach called iterative Monte Carlo procedure $[18,165]$ in their analyses.

The most recent analyses of polarized PDFs are DSSV14 [27] and NNPDFpol1.1 [16]. Motivated by the interest in assessing the impact of RHIC proton-proton data, they upgrade the corresponding previous analyses, DSSV08 [17,220] and NNPDFpol1.0 [225], with data respectively on double-spin asymmetries for inclusive jet production [226] and $\pi^{0}$ production [227] (DSSV14 ${ }^{8}$ ), and on double-spin asymmetries for high- $p_{T}$ inclusive jet production $[226,228,229]$ and single-spin asymmetries for $W^{ \pm}$ production [230] (NNPDFpol1.1). The new data have been included in NNPDFpol1.1 by means of Bayesian reweighting [231], and in DSSV14 by means of a full refit.

Overall, both the DSSV14 and NNPDFpol1.1 PDF determinations are state-of-the-art in the inclusion of the available experimental information. The data sets in the two analyses differ between

\footnotetext{
${ }^{7}$ A NNLO QCD analysis of polarized PDFs based on inclusive DIS data only was performed in Refs. [217,218]. Inclusive DIS is the only polarized process for which coefficient functions are known up to NNLO (all others are known up to NLO).

${ }^{8}$ Preliminary RHIC results included in Ref. [220] were replaced in Ref. [27] with final results.
} 


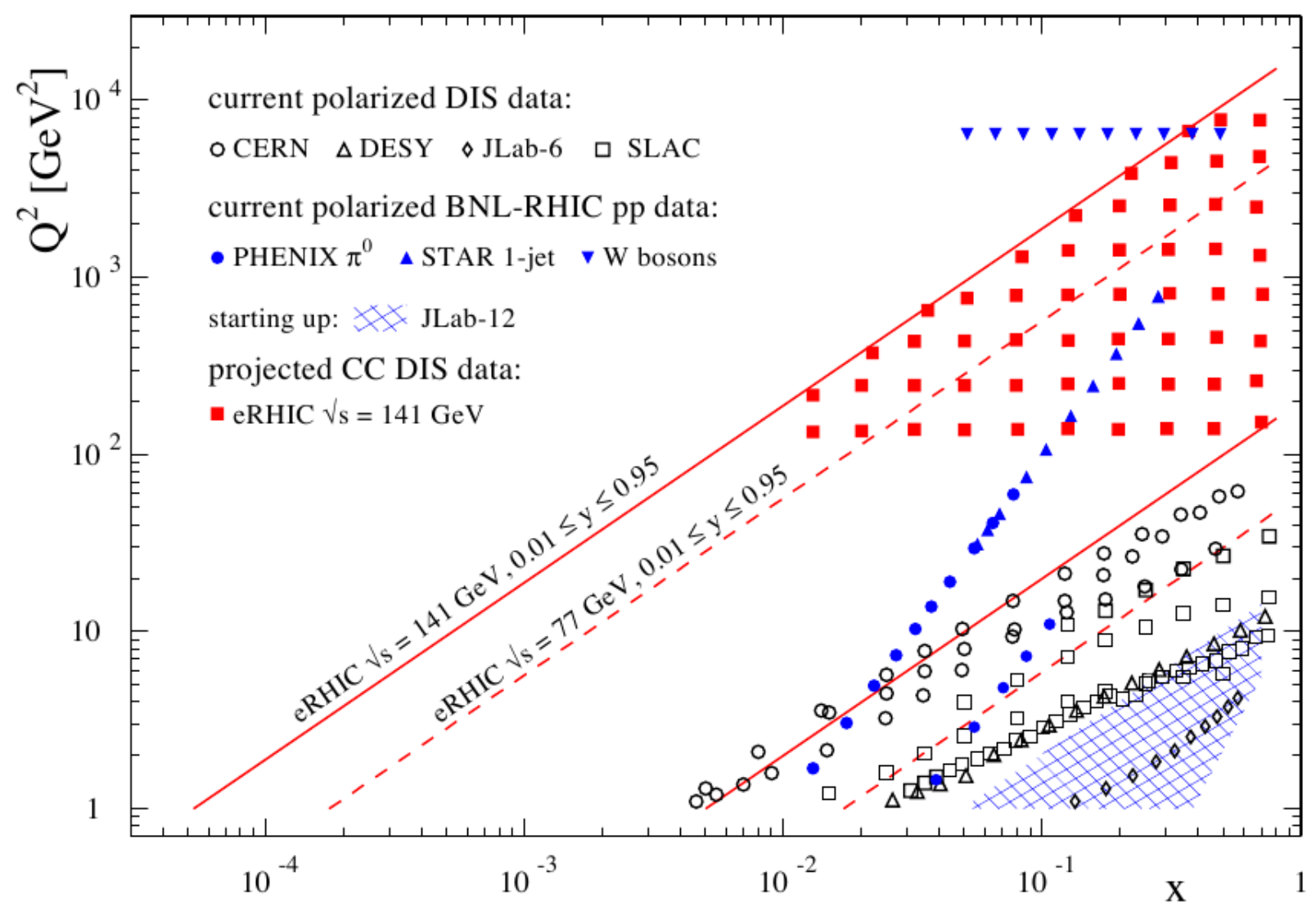

Figure 2.5: Representative kinematic coverage, in the $\left(x, Q^{2}\right)$ plane, of the (neutral current) DIS, SIDIS and proton-proton hard-scattering measurements that are used as input in a global polarized PDF fit. The extended kinematic coverage achieved by JLab-12 [214] and by an EIC [215] (including projected charged-current (CC) DIS data and denoted as eRHIC) is also shown. Figure taken from Ref. [216].

each other only in fixed-target SIDIS and RHIC $\pi^{0}$ production measurements, included in DSSV14, but not in NNPDFpol1.1. The information brought in by these data is complementary to that provided by RHIC $W^{ \pm}$production and inclusive jet production data respectively, although fraught with larger theoretical uncertainties related to fragmentation.

The effect of RHIC data on the polarized PDFs of the proton is twofold:

- The 2009 STAR and PHENIX data sets on jet and $\pi^{0}$ production [226,227], included in DSSV14 and NNPDFpol1.1, provide the first evidence of a sizable positive gluon polarization in the proton. A comparison of the gluon PDF in the two PDF sets is displayed in Fig. 2.7 (left panel). Comparable results, both central values and uncertainties, are found in the $x$ region covered by RHIC data. The agreement between the two analyses is optimal in the range $0.08 \leq x \leq 0.2$, where the dominant experimental information comes from jet data; a slightly smaller central value is found in the DSSV14 analysis, in comparison to NNPDFpol1.1, in the range $0.05 \leq x \leq 0.08$, where the dominant experimental information comes from $\pi^{0}$ production data. Indeed, these are included in DSSV14 but are not in NNPDFpol1.1. Nevertheless, best fits lie well within each other's error bands, though NNPDFpol1.1 uncertainties tend to be larger than DSSV14 uncertainties outside the region covered by RHIC data. Very consistent values of the zeroth moment of $\Delta g$, Eq. (2.6), truncated over the interval $0.05 \leq x \leq 1$, are found: at $Q^{2}=10 \mathrm{GeV}^{2}$, this is $0.20_{-0.07}^{+0.06}$ for DSSV14 [27], and $0.23 \pm 0.06$ for NNPDFpol1.1 [16]. The right plot in Fig. 2.7 shows the corresponding DSSV14 result as an example; the impact of the RHIC data is clearly visible. 

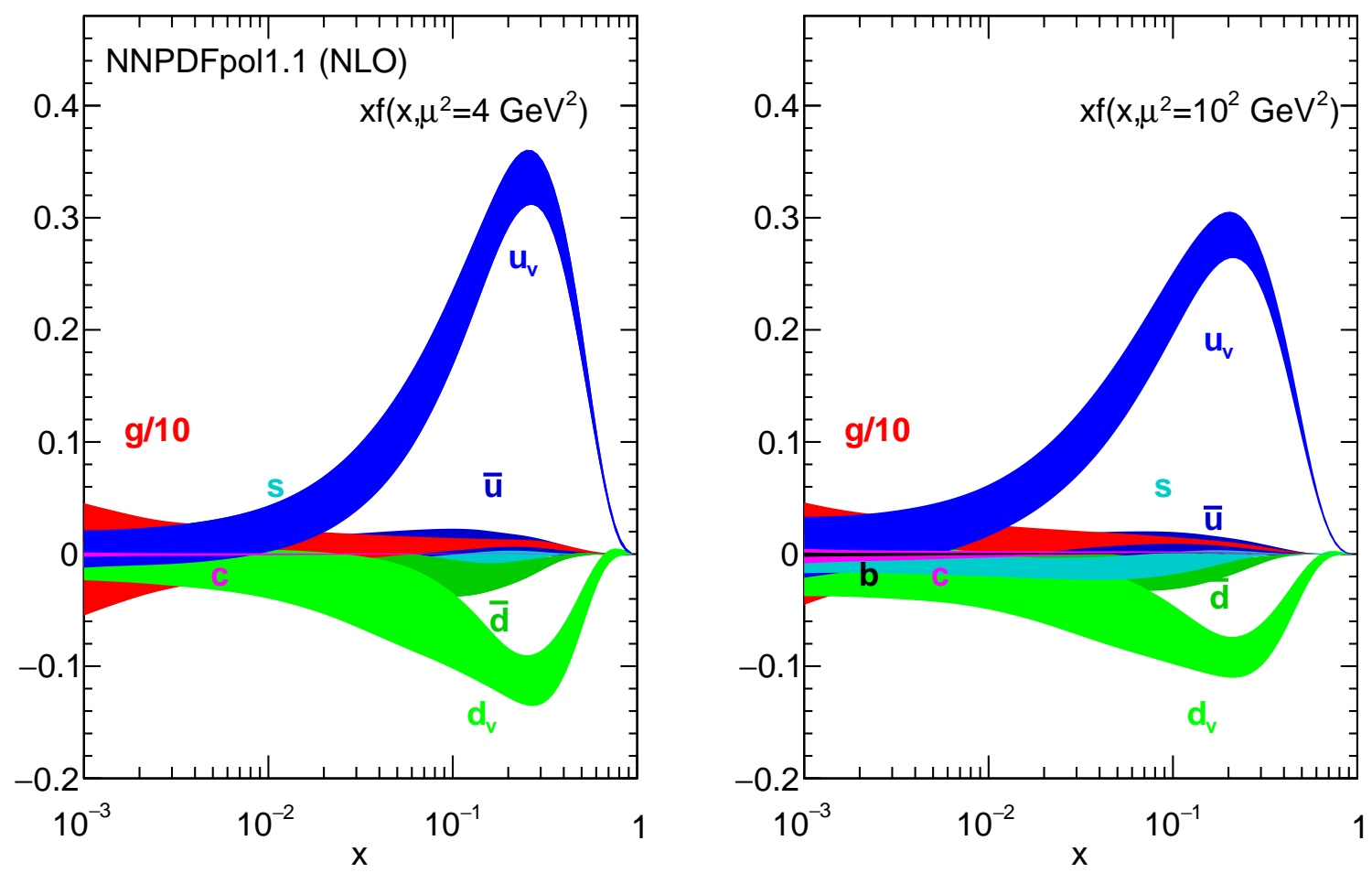

Figure 2.6: Same as Fig. 2.4, but for the polarized NNPDFpol1.1 NLO PDFs [16].
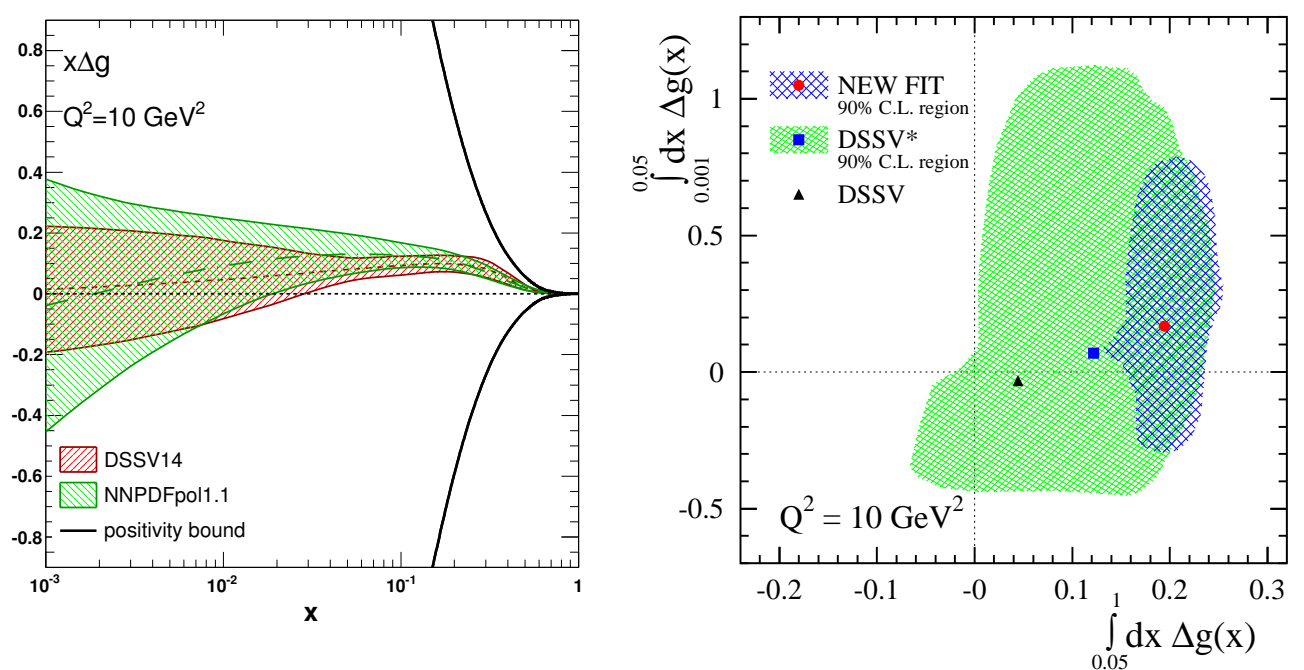

Figure 2.7: (Left) The polarized gluon momentum distribution $x \Delta g$ from the DSSV14 (with 90\% C.L. uncertainty band) and NNPDFpol1.1 PDF sets at $Q^{2}=10 \mathrm{GeV}^{2}$. The NNPDF3.1 positivity bound is also shown. (Right) $90 \%$ C.L. areas in the plane spanned by the truncated moments of $\Delta g$ computed for $0.05 \leq x \leq 1$ and $0.001 \leq x \leq 0.05$ at $Q^{2}=10 \mathrm{GeV}^{2}[27]$

- The 2012 STAR data sets on $W$ production [230], included in NNPDFpol1.1, provide evidence of a positive $\Delta \bar{u}$ distribution and a negative $\Delta \bar{d}$ distribution, with $|\Delta \bar{d}|>|\Delta \bar{u}|[16]$. The size of the flavor symmetry breaking for polarized sea quarks is quantified by the asymmetry $\Delta \bar{u}-\Delta \bar{d}$, which, in the NNPDFpol1.1 analysis, turn out to be roughly as large as its unpolarized counterpart (in absolute value) [11], though much more uncertain [232]. Even within this uncertainty, polarized 
and unpolarized light sea quark asymmetries show opposite signs, with the polarized one being clearly positive. This trend is also found from analysis of the polarized SIDIS data, as revealed by the DSSV08 parton set. This result may discriminate among various models of nucleon structure, see [232] and references therein.

Open issues. Despite the achievements described above, the polarized PDFs presently cannot be determined in a global QCD analysis with the same accuracy as their unpolarized counterparts. The experimental data are confined to a relatively narrow range of $x$ and $Q^{2}$. As a consequence, the size of the contributions of quarks, antiquarks and gluons to the nucleon spin, as quantified by their zeroth moments, Eq. (2.6), are still affected by large uncertainties. These come predominantly from the extrapolation into the small- $x$ region $\left(x \lesssim 10^{-3}\right)$. Here potential modifications in the PDF shape induced by small- $x$ evolution [233-239] could arise, which presently cannot be tested. Significant uncertainties also affect the PDFs in the large- $x$ valence region $(x \gtrsim 0.7)$. This regime is less relevant for the determination of the PDF moments, but it is important for comparisons to nonperturbative models of nucleon structure, especially in terms of ratios of light-quark polarized to unpolarized PDFs (for a comparison between large- $x$ PDFs and model predictions, see Ref. [162]). Finally, the small lever arm of the data in $Q^{2}$ is a serious limiting factor in the determination of $\Delta g$ via evolution, unless the data at low $Q^{2}$ and large $x$ are included in the fit and carefully analyzed. This requires an appropriate treatment of power-suppressed corrections and possibly a minimization methodology which can iteratively focus on a region in parameter space where constraints are not too strong, as done in the JAM15 analysis [18].

The determination of the total polarized strange distribution $\Delta s^{+}$is also particularly delicate. Inclusive DIS data, together with nonsinglet axial couplings, Eq. (2.51), and kaon SIDIS data provide the sole available constraint on $\Delta s^{+}$. A sizable negative $\Delta s^{+}$is found consistently in all analyses based on inclusive DIS data only, as a result of the constraint from hyperon decays that is usually adopted. However, the shape of $\Delta s^{+}$may change significantly in analyses that also include SIDIS data. Typically SIDIS data lead to a trend for $\Delta s^{+}$to be small or even slightly positive in the medium $x$-range, although this depends also on the set of kaon FFs used to compute the corresponding observables [240]. The recent study in Ref. [165] sheds some light on this issue by performing a simultaneous determination of polarized PDFs and unpolarized FFs using DIS, SIDIS and single-inclusive annihilation data. In order to avoid biasing the determination of $\Delta s^{+}$by assumptions on $\mathrm{SU}(3)$ symmetry, the octet axial charge in Eq. (2.51) has been allowed to be determined by the data alone. As a consequence, a slightly positive $\Delta s^{+}$distribution, but compatible with the negative result found from inclusive DIS within its large uncertainties, has been obtained. An octet axial charge about $20 \%$ smaller than its quoted experimental value, Eq. (2.51), appears to be preferred by the data. This implies a zeroth moment $\langle 1\rangle_{\Delta s^{+}}=-0.03 \pm 0.1$ at $\mu^{2}=1 \mathrm{GeV}^{2}$, and hence a larger $\Delta \Sigma$, Eq. (2.5), than in most other present analyses. However, we stress that the determination of $\Delta s^{+}$from SIDIS data also relies on good knowledge of the unpolarized strange distribution. Furthermore, unpolarized SIDIS data themselves set constraints on FFs and ultimately need to be included as well to obtain a reliable picture [210]. In any case, further higher precision kaon SIDIS data will be needed to reduce the uncertainty on $\Delta s^{+}$ and further test the degree of $\mathrm{SU}(3)$ breaking.

Ongoing and future experimental campaigns at current facilities are expected to provide additional experimental information useful to clarify some of the issues outlined above (for an assessment of the impact of very recent/forthcoming data, see e.g. Refs. [211,241-243]). However, a future high-energy, polarized EIC [215] will likely be the only facility to be able to address all of the above issues with the highest precision. The extension of the kinematic reach down to $x \sim 10^{-4}$ and up to $Q^{2}=10^{4} \mathrm{GeV}^{2}$ will allow for an accurate determination of $\Delta g$ via evolution in DIS/SIDIS, of $\Delta \bar{u}$ and $\Delta \bar{d}$ via inclusive DIS at high $Q^{2}$ mediated by electroweak bosons, and of $\Delta s$ via kaon-tagged SIDIS. The potential impact of the longitudinally polarized program at an EIC has been quantitatively assessed in several dedicated studies [241,244-246]. 


\section{Benchmarking PDF moments}

In this section we provide a quantitative comparison between current lattice-QCD and global-fit results of the lowest moments of unpolarized and polarized PDFs. To this purpose, we identify benchmark quantities and define the criteria to appraise the determinations available in the literature. For each benchmark quantity, we specify a prescription to select and combine lattice-QCD calculations and global-fit determinations. We present our benchmark numbers from each side and compare them.

\subsection{Benchmark criteria}

We start by describing our benchmark criteria, which include the definition of the benchmark quantities and the determination of their reference values, based on a careful assessment of the lattice-QCD and global-fit results available in the literature.

\subsubsection{Benchmark quantities}

We identify our benchmark quantities with the following moments of unpolarized and polarized PDFs, or of PDF quark flavor combinations.

- $\langle x\rangle_{u^{+-d^{+}}},\langle x\rangle_{u^{+}},\langle x\rangle_{d^{+}},\langle x\rangle_{s^{+}}$and $\langle x\rangle_{g}$ in the unpolarized case;

- $g_{A} \equiv\langle 1\rangle_{\Delta u^{+}-\Delta d^{+}},\langle 1\rangle_{\Delta u^{+}},\langle 1\rangle_{\Delta d^{+}},\langle 1\rangle_{\Delta s^{+}}$and $\langle x\rangle_{\Delta u^{-}-\Delta d^{-}}$in the polarized case.

We adopt the conventional notation described in Appendix A. We focus on the above quantities because current lattice calculations of higher moments and moments of other PDF combinations are not sufficiently controlled to allow for a meaningful comparison between lattice-QCD and global-fit results.

\subsubsection{Appraising lattice-QCD calculations}

To accurately assess current lattice-QCD calculations available in the literature, we follow a procedure inspired by the review of low-energy mesons undertaken by the Flavor Lattice Averaging Group (FLAG) [81]. For each lattice calculation, we characterize each source of uncertainty outlined in Sec. 2.2. We use a rating system inspired by FLAG, awarding a blue star $(\star$ ) for sources of uncertainty that are well controlled or very conservatively estimated, a blue circle (O) for sources of uncertainty that have been controlled or estimated to some extent, and a red square ( $\boldsymbol{\square})$ for uncertainties that have not met our criteria or for which no estimate is given. Specifically, the rating system works as follows.

- Discretization effects and the continuum limit. We assume that the lattice actions are $\mathcal{O}(a)$-improved, i.e., that the discretization errors vanish quadratically with the lattice spacing. For unimproved actions, an additional lattice spacing is required. These criteria must be satisfied in each case for at least one pion mass below $300 \mathrm{MeV}$.

At least three lattice spacings with at least two lattice spacings below $0.1 \mathrm{fm}$ and a range of lattice spacings that satisfies $\left[a_{\max } / a_{\min }\right]^{2} \geq 2$.

$\bigcirc$ At least two lattice spacings with at least one point below $0.1 \mathrm{fm}$ and a range of lattice spacings that satisfy $\left[a_{\max } / a_{\min }\right]^{2} \geq 1.4$.

To receive a $\star$ or $\bigcirc$ either a continuum extrapolation must be performed, or the results must demonstrate no significant discretization effects over the appropriate range of lattice spacings.

- Unphysical pion masses. We define a physical pion mass ensemble to be one with $M_{\pi}=$ $135 \pm 10 \mathrm{MeV}$ for the following criteria. 
$\star$ One ensemble with a physical pion mass or a chiral extrapolation with three or more pion masses, with at least two pion masses below $250 \mathrm{MeV}$ and at least one below $200 \mathrm{MeV}$.

O A chiral extrapolation with three or more pion masses, two of which are below $300 \mathrm{MeV}$.

- Finite-volume effects. For calculations that use a mixed-action approach, i.e., with different lattice actions for the valence and sea quarks, we apply the following criteria to $M_{\pi} L$ for the valence quarks $\left(M_{\pi, \min }\right.$ is the lightest pion mass employed in the calculation).

$\star$ Ensembles with $M_{\pi, \min } L \geq 4$, or at least three volumes with spatial extent $L>2.5 \mathrm{fm}$.

○ Ensembles with $M_{\pi, \min } L \geq 3.4$, or at least two volumes with spatial extent $L>2.5 \mathrm{fm}$.

\section{- Excited-state contamination.}

At least three source-sink separations or a variational method to optimize the operator derived from at least a $3 \times 3$ correlator matrix, at every pion mass and lattice spacing.

O Two source-sink separations at every pion mass and lattice spacing, or three or more sourcesink separations at one pion mass below $300 \mathrm{MeV}$. For the variational method, an optimized operator derived from a $2 \times 2$ correlator matrix at every pion mass and lattice spacing, or a $3 \times 3$ correlator matrix for one pion mass below $300 \mathrm{MeV}$.

\section{- Renormalization.}

$\star$ Nonperturbative renormalization.

○ Perturbative renormalization.

For $g_{A}$ we also award a $\star$ for calculations that use fermion actions for which $Z_{A} / Z_{V}=1$ or employ combinations of quantities for which the renormalization is unity by construction.

- Lattice-spacing determination. For lattice-QCD calculations of nucleons, the lattice-spacing determination is generally sufficiently precise that it is a very small or negligible source of systematic uncertainty. Therefore we do not include an assessment of the lattice-spacing determination in our criteria.

Another important parameter in lattice-QCD calculations is the number of sea quark flavors, $N_{f}$. Following the approach used by FLAG, we prefer to avoid combining calculations with differing $N_{f}$; for more discussion of this issue, see the FLAG review [81].

We now summarize the current status of lattice-QCD calculations of our benchmark moments of unpolarized and polarized PDFs respectively. Following FLAG, we consider only those results that are published in peer-reviewed journals or that have appeared as preprints. Where recent results are a clear update of previously published work, we do not include earlier results. A bibliographical compilation of the results available in the literature is given for completeness in Appendix B, Tables B.5-B.10. We characterize the results according to the criteria described above, and provide a prescription to combine those results that satisfy the criteria into a single benchmark value.

Our criteria and the corresponding ratings are chosen to provide not only as fair an assessment of the relative merits of various calculations as possible, but also a solid reference for future studies. Where lattice-QCD results do not meet these standards, we hope that the lattice community will work towards improved calculations and greater precision. 


\begin{tabular}{|c|c|c|c|c|c|c|c|c|c|c|c|}
\hline Mom. & Collab. & Ref. & $N_{f}$ & Status & Disc & $\mathrm{QM}$ & $\mathrm{FV}$ & Ren & ES & & Value \\
\hline \multirow[t]{3}{*}{$\langle x\rangle_{u^{+}-d^{+}}$} & LHPC 14 & {$[247]$} & $2+1$ & $\mathrm{P}$ & ש & $\star$ & $\star$ & $\star$ & $\star$ & & $0.140(21)$ \\
\hline & ETMC 17 & [248] & 2 & $\mathrm{P}$ & - & $\star$ & - & $\star$ & $\star$ & * & $0.194(9)(11)$ \\
\hline & RQCD 14 & [249] & 2 & $\mathrm{P}$ & - & $\boldsymbol{\square}$ & O & $\star$ & $\star$ & ** & $0.217(9)$ \\
\hline$\langle x\rangle_{u^{+}}$ & ETMC 17 & [248] & 2 & $\mathrm{P}$ & $\mathbf{\square}$ & $\star$ & - & $\star$ & $\star$ & $* \triangleright$ & $0.453(57)(48)$ \\
\hline$\langle x\rangle_{d^{+}}$ & ETMC 17 & [248] & 2 & $\mathrm{P}$ & 口 & $\star$ & - & $\star$ & $\star$ & $* \triangleright$ & $0.259(57)(47)$ \\
\hline$\langle x\rangle_{s^{+}}$ & ETMC 17 & [248] & 2 & $\mathrm{P}$ & $\boldsymbol{\square}$ & $\star$ & - & $\star$ & $\star$ & $* \triangleright$ & $0.092(41)(0)$ \\
\hline$\langle x\rangle_{g}$ & ETMC 17 & [248] & 2 & $\mathrm{P}$ & - & $\star$ & च & O & $\star$ & * & $0.267(22)(27)$ \\
\hline
\end{tabular}

* Study employing a single physical pion mass ensemble.

** Study employing a single ensemble with $m_{\pi}=150 \mathrm{MeV}$.

$\triangleright$ Nonsinglet renormalization is applied.

Table 3.1: Status of current lattice-QCD calculations of the benchmark first moments of unpolarized PDFs listed in Sec. 3.1.1. A detailed description of each entry, including the symbols used to characterize the various sources of systematics, is provided in the text. Values are shown at $\mu^{2}=4 \mathrm{GeV}^{2}$. We refer the reader to the corresponding references for details on the errors reported in parentheses. To denote the various sources of systematic uncertainty, we use the abbreviations Disc (discretization), QM (quark mass), FV (finite volume), Ren (renormalization) and ES (excited states).

Unpolarized parton distributions. We summarize the current status of lattice-QCD calculations of the benchmark moments of unpolarized PDFs listed in Sec. 3.1.1 in Table 3.1. We indicate: the computed moment in the first column; the collaboration who performed the computation in the second column; the corresponding reference in the third column; the number of sea quark flavors, $N_{f}$, in the fourth column. We show whether the calculation has been published (P) or has appeared as a preprint (PreP) in the fifth column. In the following five columns, we assess each source of systematic uncertainty according to the criteria listed above. In the last column, we report the computed value at $\mu^{2}=4 \mathrm{GeV}^{2}$ in the $\overline{\mathrm{MS}}$ scheme. We refer the reader to the corresponding references for details on the meaning of the errors reported in parentheses. We do not list results that have not been extrapolated to the physical pion mass, nor do we include quenched results in Table 3.1. For completeness, we report these results in Appendix B, Table B.1.

As is apparent from Table 3.1, there are no lattice calculations of the considered first moments for which all systematics have been fully explored and controlled. In the case of $\langle x\rangle_{u^{+}-d^{+}}$three different results are available in the literature. We present the lattice-QCD benchmark value for this quantity as a best-estimate band. This band extends from the mean of the smallest result minus its error to the mean of the largest result plus its error, and includes all results listed in Table 3.1 with two or more sea quark flavors. Current studies are not sufficiently precise to distinguish between results with different numbers of sea quark flavors. In the case of $\langle x\rangle_{u^{+}},\langle x\rangle_{d^{+}},\langle x\rangle_{s^{+}}$and $\langle x\rangle_{g}$, there is only one lattice result available in the literature: for these quantities, our lattice-QCD benchmark value is the single result; however, it should be noted that these results may underestimate some sources of uncertainty.

The lattice-QCD benchmark numbers for $\langle x\rangle_{u^{+}-d^{+}},\langle x\rangle_{u^{+}},\langle x\rangle_{d^{+}},\langle x\rangle_{s^{+}}$and $\langle x\rangle_{g}$ will be further commented below, where they will be collected together with their global-fit counterparts in Table 3.7.

Finally, we summarize the current status of lattice-QCD calculations of the second moment of the unpolarized valence-quark PDFs, $\left\langle x^{2}\right\rangle_{u^{-}},\left\langle x^{2}\right\rangle_{d^{-}}$and $\left\langle x^{2}\right\rangle_{u^{-}-d^{-}}$in Appendix B, Table B.2. The study of these moments is not sufficiently mature to provide benchmark values and we only list the results for completeness. 


\begin{tabular}{|c|c|c|c|c|c|c|c|c|c|c|c|}
\hline Mom. & Collab. & Ref. & $N_{f}$ & Status & Disc & $\mathrm{QM}$ & $\mathrm{FV}$ & Ren & ES & & Value \\
\hline \multirow[t]{7}{*}{$g_{A}$} & CalLat 17 & {$[250]$} & $2+1+1$ & PreP & $\mathbf{\square}$ & $\star$ & $\mathbf{\square}$ & $\star$ & $\star$ & & $1.278(21)(26)$ \\
\hline & PNDME 16 & {$[251]$} & $2+1+1$ & $\mathrm{P}$ & O & $\star$ & O & $\star$ & $\star$ & & $1.195(33)(20)$ \\
\hline & LHPC 14 & {$[247]$} & $2+1$ & $\mathrm{P}$ & $\square$ & $\star$ & $\star$ & $\star$ & $\star$ & & $0.97(8)$ \\
\hline & Mainz 17 & {$[252]$} & 2 & PreP & $\star$ & 0 & $\star$ & $\star$ & $\star$ & & $1.278(68)\left({ }_{-0.087}^{+0}\right)$ \\
\hline & ETMC 17 & {$[253]$} & 2 & $\mathrm{P}$ & $\mathbf{a}$ & $\star$ & $\mathbf{a}$ & $\star$ & $\star$ & $*$ & $1.212(33)(22)$ \\
\hline & RQCD 15 & {$[254]$} & 2 & $\mathrm{P}$ & O & O & O & $\star$ & O & $\ddagger$ & $1.280(44)(46)$ \\
\hline & QCDSF 14 & {$[255]$} & 2 & $\mathrm{P}$ & 0 & 0 & O & $\star$ & $\mathbf{\square}$ & $\ddagger$ & $1.29(5)(3)$ \\
\hline
\end{tabular}

* Study employing a single physical pion mass ensemble.

$g_{A}$ is determined via the ratio $g_{A} / f_{\pi}$, employing the physical value for $f_{\pi}$.

Table 3.2: Same as Table 3.1, but for the axial coupling, $g_{A} \equiv\langle 1\rangle_{\Delta u^{+}-\Delta d^{+}}$. Studies with three or more red squares are omitted from this table. Values are shown at $\mu^{2}=4 \mathrm{GeV}^{2}$.

Polarized parton distributions. The zeroth moment of the isotriplet polarized PDF combination is related to the axial charge of the nucleon, $g_{A} \equiv\langle 1\rangle_{\Delta u^{+}-\Delta d^{+}}$. This quantity is of central importance to nucleon physics and has long been considered an important benchmark for lattice calculations. Historically, lattice-QCD calculations of the axial charge have underestimated the experimental value $g_{A}^{\exp }=1.2723(23)[28]$ (see also Eq. (2.50)), which is most precisely determined from neutron weak decays. Thus, the axial charge has been the single most-studied moment in lattice QCD. We summarize the current status of these calculations in Table 3.2 using the same format as in Table 3.1. All results are quoted at $\mu^{2}=4 \mathrm{GeV}^{2}$.

As is apparent from Table 3.2, we consider only three calculations of $g_{A}$ to have all systematics sufficiently controlled to obtain a blue circle or star. One of them [251] is for $N_{f}=2+1+1$, while two of them $[252,254]$ are for $N_{f}=2$. In the former case, our benchmark value corresponds to the single calculation; in the latter case, our benchmark value corresponds to a weighted average of [252] and [254], assuming correlations between the results, and applying the procedure of [256]. In summary, our benchmark values are

$$
g_{A}^{N_{f}=2+1+1}=1.195(33)(20), \quad \text { and } \quad g_{A}^{N_{f}=2}=1.279(50) .
$$

We observe that the result of [250], although it does not fulfill all our requirements on systematic uncertainties, uses the same gauge configurations as those of [251]. Therefore, we also carry out a simultaneous fit to the two results for completeness. We use a fit function of the form

$$
\begin{aligned}
g_{A}^{\mathrm{fit}} & =c_{0}+f(a)+c_{3} M_{\pi}^{2}+c_{4} M_{\pi}^{2} \exp \left(-M_{\pi} L\right)+c_{5} M_{\pi}^{2} \log \left(\frac{M_{\pi}^{2}}{\Lambda_{\chi \mathrm{PT}}^{2}}\right), \\
f(a) & =\left\{\begin{array}{ll}
c_{1} a & \text { Ref. }[251] \\
c_{2} a^{2} & \text { Ref. [250] }
\end{array} .\right.
\end{aligned}
$$

The coefficient $c_{1}$ captures $\mathcal{O}(a)$ effects present in the valence-quark action of [251], while [250] has discretization effects starting at $\mathcal{O}\left(a^{2}\right)$. The term proportional to $c_{4}$ captures the leading finite-volume effects, and $c_{3}$ and $c_{5}$ represent chiral-extrapolation terms. Modifications to this fit form, including setting $c_{5}=0$, have a negligible effect on the fit results within extrapolation uncertainties, and the final result is in very good agreement with a weighted average of the two calculations, assuming $100 \%$ correlations, which is $g_{A}^{N_{f}=2+1+1, \text { avg }}=1.243(36)$. Based on this fit, we find the best-estimate band of

$$
g_{A}^{N_{f}=2+1+1, \text { iit }}=1.22-1.28 \text {. }
$$




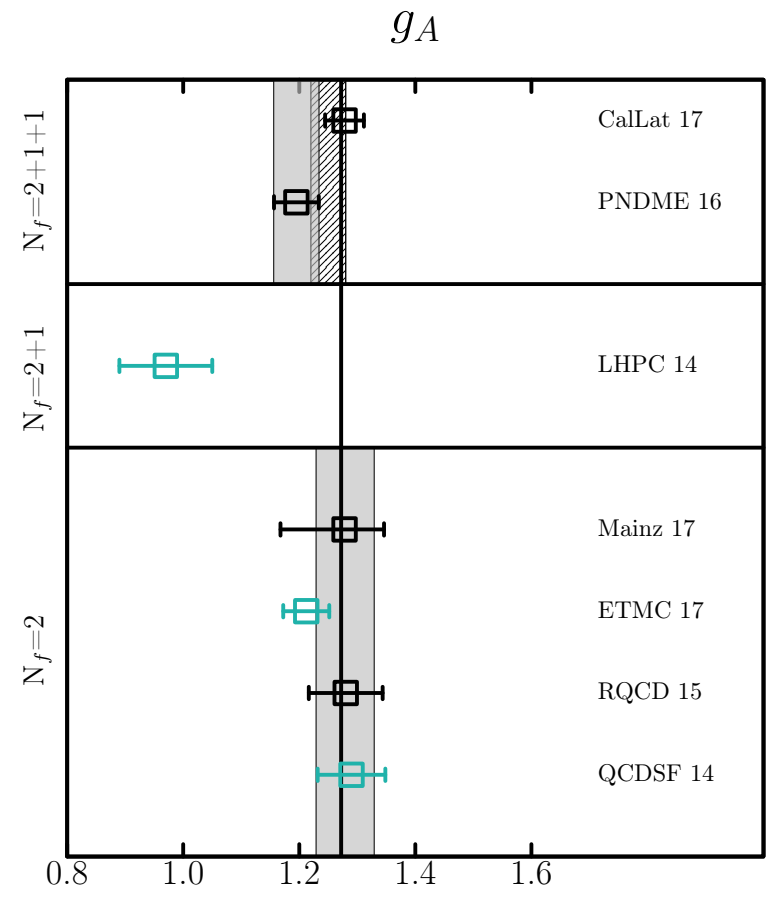

Figure 3.1: Summary of the current status of lattice-QCD calculations of the axial charge, $g_{A} \equiv\langle 1\rangle_{\Delta u^{+}-\Delta d^{+}}$. The vertical black line represents the current experimental world average $g_{A}^{\exp }=1.2723(23)$ [28]. The light gray bands for $N_{f}=2+1+1$ and $N_{f}=2$ represent the benchmark results of Eq. (3.1), and the dashed gray band for $N_{f}=2+1+1$ is the fit band of Eq. (3.4).

We plot all lattice results for the axial coupling, listed in Table 3.2, in Fig. 3.1. We show the world-average experimental value as a vertical black line. The light gray bands for $N_{f}=2+1+1$ and $N_{f}=2$ represent the benchmark results of Eq. (3.1), and the dashed gray band for $N_{f}=2+1+1$ is the combined fit band given in Eq. (3.4).

In addition to the axial charge, we summarize the zeroth moments of the individual light-quark total polarized distributions in Table 3.3. We summarize the status of lattice-QCD calculations of the first moments of the polarized PDF combination $\langle x\rangle_{\Delta u^{-}-\Delta d^{-}}$in Table 3.4. We use the same format as in Table 3.1. All values are at $\mu^{2}=4 \mathrm{GeV}^{2}$. Available results that have not been extrapolated to the physical pion mass or quenched results are not reported here, but in Appendix B, Tables B.3-B.4, for completeness.

In the case of $\langle 1\rangle_{\Delta u^{+}}$and $\langle 1\rangle_{\Delta d^{+}}$, there is only one result available in the literature for each quantity. Therefore, although the corresponding systematic uncertainties are not completely under control and possibly underestimated, we take the individual results as our benchmark values. In the case of $\langle 1\rangle_{\Delta s^{+}}$ and $\langle x\rangle_{\Delta u^{-}-\Delta d^{-}}$, however, several results are available in the literature, although without a full characterization of their systematic uncertainties. We present our lattice-QCD benchmark value for these quantities as a best-estimate band extending from the mean minus the error of the smallest result to the mean plus the error of the largest. We include all results with two or more flavors of sea quarks listed in Tables 3.3 and 3.4, respectively.

The lattice-QCD benchmark numbers for $g_{A},\langle 1\rangle_{\Delta u^{+}},\langle 1\rangle_{\Delta d^{+}},\langle 1\rangle_{\Delta s^{+}}$and $\langle x\rangle_{\Delta u^{-}-\Delta d^{-}}$will be further commented below, where they will be collected together with their global-fit counterparts in Table 3.8.

\subsubsection{Appraising global-fit results}

The current status of global PDF fit determinations and their uncertainties has been carefully assessed in dedicated reviews recently $[5,6]$, and further summarized in Sec. 2.3.2. It is now recognized that PDF 


\begin{tabular}{|c|c|c|c|c|c|c|c|c|c|c|c|}
\hline Mom. & Collab. & Ref. & $N_{f}$ & Status & Disc & $\mathrm{QM}$ & $\mathrm{FV}$ & Ren & ES & & Value \\
\hline$\langle 1\rangle_{\Delta u^{+}}$ & ETMC 17 & [248] & 2 & $\mathrm{P}$ & - & $\star$ & 口 & $\star$ & $\star$ & * & $0.830(26)(4)$ \\
\hline$\langle 1\rangle_{\Delta d^{+}}$ & ETMC 17 & [248] & 2 & $\mathrm{P}$ & - & $\star$ & घ & $\star$ & $\star$ & $*$ & $-0.386(16)(6)$ \\
\hline \multirow[t]{3}{*}{$\langle 1\rangle_{\Delta s^{+}}$} & $\chi \mathrm{QCD} 17$ & [257] & $2+1$ & $\mathrm{P}$ & [ & 0 & 0 & $\star$ & $\star$ & $\dagger, \triangleleft$ & $-0.0403(44)(78)$ \\
\hline & Engelhardt 12 & {$[258]$} & $2+1$ & $\mathrm{P}$ & - & $\mathbf{\square}$ & o & $\star$ & $\star$ & $\triangleleft$ & $-0.031(17)$ \\
\hline & ETMC 17 & [248] & 2 & $\mathrm{P}$ & [ & $\star$ & [ & $\star$ & $\star$ & * & $-0.042(10)(2)$ \\
\hline
\end{tabular}

* Study employing a single physical pion mass ensemble.

$\dagger$ Partially quenched simulation with $m_{\pi}=330 \mathrm{MeV}$. Criteria applied to the valence quarks.

$\triangleleft$ Some parts of the renormalization are estimated, see references for details.

Table 3.3: Same as Table 3.1, but for the zeroth moments of the polarized total quark distributions. Values are shown at $\mu^{2}=4 \mathrm{GeV}^{2}$.

\begin{tabular}{|c|c|c|c|c|c|c|c|c|c|c|}
\hline Mom. & Collab. & Ref. & $N_{f}$ & Status & Disc & QM & FV & Ren & ES & Value \\
\hline \multirow[t]{3}{*}{$\langle x\rangle_{\Delta u^{-}-\Delta d^{-}}$} & $\begin{array}{l}\mathrm{RBC} / \\
\mathrm{UKQCD} 10\end{array}$ & [259] & $2+1$ & $\mathrm{P}$ & 口 & $\square$ & $\star$ & $\star$ & 口 & $\begin{array}{l}0.256(23) / \\
0.205(59)\end{array}$ \\
\hline & LHPC 10 & [260] & $2+1$ & $\mathrm{P}$ & 口 & $\square$ & O & O & 口 & $0.1972(55)$ \\
\hline & ETMC 15 & [261] & 2 & $\mathrm{P}$ & च & $\star$ & [ & $\star$ & $\star$ & $0.229(33)$ \\
\hline
\end{tabular}

* Study employing a single physical pion mass ensemble.

Table 3.4: Same as Table 3.1, but for the first moment of the polarized valence-quark distribution. Values are shown at $\mu^{2}=4 \mathrm{GeV}^{2}$.

uncertainties receive various contributions: the measurement uncertainty propagated from the data, uncertainties associated with incompatible data sets, procedural uncertainties such as those related to the choice of the PDF parametrization, and the handling of systematic errors, among others. As outlined in Sec. 2.3.2, in principle all of these uncertainties can be accounted for with suitable methods, both in the Hessian and the MC frameworks. In practice, there is a significant spread in the sophistication of these methods between unpolarized and polarized PDF fits.

In Sec. 2.3.2, we also emphasized that there are additional theoretical uncertainties on PDFs associated with uncertainty in the input values of the physical parameters used in the fit (such as the reference value of the strong coupling) and with missing higher-order uncertainties (given that fits are usually performed with fixed-order perturbation theory). The size of the former can be accounted for by studying the stability of the results upon variation of the input parameters; the size of the latter is currently unknown, although it is supposed to be sub-dominant. Therefore, theoretical uncertainties will not be considered in the following.

As far as full moments of PDFs are concerned, global-fit results involve some degree of extrapolation to the region not covered by experimental data, that is not necessarily well accounted for in the PDF error estimates. Extrapolation is particularly delicate to small $x$ values in the case of polarized PDFs: opposite to unpolarized PDFs, the kinematic coverage is fairly limited (see Sec. 2.3 .3 and in particular Fig. 2.5) and there is no analog of the momentum sum rule, Eq. (2.49), to further constrain the PDFs. Extrapolation uncertainties are difficult to quantify, unless one naively extrapolates uncertainty bands from the measured region.

We now summarize the results for our benchmark moments listed in Sec. 3.1.1, based on current global-fit determinations of unpolarized and polarized PDFs. We specify how the available results are 


\begin{tabular}{lcccccc}
\hline Mom. & NNPDF3.1 & CT14 & MMHT2014 & ABMP2016 & CJ15 & HERAPDF2.0 \\
\hline$\langle x\rangle_{u^{+}-d^{+}}$ & $0.152(3)$ & $0.158(4)$ & $0.151(4)$ & $0.167(4)$ & $0.152(2)$ & $0.188(3)$ \\
$\langle x\rangle_{u^{+}}$ & $0.348(4)$ & $0.348(3)$ & $0.348(5)$ & $0.353(3)$ & $0.348(1)$ & $0.372(4)$ \\
$\langle x\rangle_{d^{+}}$ & $0.196(3)$ & $0.190(3)$ & $0.197(5)$ & $0.186(3)$ & $0.196(1)$ & $0.185(7)$ \\
$\langle x\rangle_{s^{+}}$ & $0.039(3)$ & $0.035(5)$ & $0.035(9)$ & $0.041(2)$ & - & $0.035(11)$ \\
$\langle x\rangle_{g}$ & $0.410(4)$ & $0.416(5)$ & $0.411(9)$ & $0.412(4)$ & $0.416(1)$ & $0.401(10)$ \\
\hline
\end{tabular}

Table 3.5: Status of current global PDF fit determinations of the benchmark moments of unpolarized PDFs listed in Sec. 3.1.1. All values are shown at $\mu^{2}=4 \mathrm{GeV}^{2}$. See text for details about the calculation of PDF uncertainties in each case.

combined into a single benchmark value.

Unpolarized parton distributions. We summarize the current status of global-fit results of the benchmark moments of unpolarized PDFs listed in Sec. 3.1.1 in Table 3.5. In the first column we indicate the computed moment, and in the subsequent six columns the moment's value, obtained from the most recent available PDF determinations: NNPDF3.1 [11], CT14 [13], MMHT2014 [12], ABMP16 [14] (with $N_{f}=4$ flavors), CJ15 [15] and HERAPDF2.0 [20] respectively. The most relevant features of these PDF sets have been presented in Sec. 2.3.2. All values in Table 3.5 are displayed at $\mu^{2}=4 \mathrm{GeV}^{2}$. They have been obtained from the default PDF sets at the highest available perturbative order, which is NNLO for all of them except CJ15 for which it is NLO. The uncertainties for the CT14 PDF set has been rescaled by a factor $1 / 1.65$ to convert from $90 \%$-CL bands to $68 \%$-CL bands. Note that tolerance of $\Delta \chi^{2}=1$ at $68 \%$ CL is used in the CJ15 PDF set; hence, the smaller uncertainties of this set compared to all the other PDF sets. Also, the CJ15 set does not fit $\langle x\rangle_{s^{+}}$, therefore the corresponding number is not displayed in Table 3.5. In the case of the HERAPDF2.0 set, the error band is the sum in quadrature of the statistical, model and parametrization uncertainties. Taking the results of Table 3.5 at face value, there are clear discrepancies arising from a variety of factors [8,9]; we examine some of these in the following.

In order to provide a benchmark value for the first moments of unpolarized PDFs listed in Table 3.5, we follow the latest PDF4LHC 2015 recommendations [8]. Even though the recommendations were primarily formulated for the usage of PDFs in LHC-related physics, and alternative recommendations have been suggested [9], we find it useful to apply them here as well. The reason is twofold. First, this benchmark exercise aims at accuracy and precision, two of the guiding principles underlying the recommendations. Second, they led to the release of a specific PDF set that can be easily used to compute all the needed benchmark values.

While we refer the reader to [8] for details, here we only mention that the PDF4LHC15 PDF set was constructed by means of a statistical combination [178,180,181,200] (an unweighted average) of the NNPDF3.0 [262], CT14 and MMHT2014 PDF sets. ${ }^{9}$ The three PDF sets were selected among all the publicly available PDF sets based on four criteria [8].

- A global data set from a wide variety of observables and processes should be included in the fit analysis.

- Theoretical hard cross sections should be evaluated up to NNLO in a general-mass variable-flavor number scheme with up to $N_{f}^{\max }=5$ active quark flavors.

\footnotetext{
${ }^{9}$ The NNPDF3.1 PDF set was not available when the recommendations were formulated.
} 


\begin{tabular}{lcccc}
\hline Mom. & NNPDFpol1.1 & DSSV08 & JAM15 & JAM17 \\
\hline$\langle 1\rangle_{\Delta u^{+}-\Delta d^{+}}$ & $1.250(16)$ & $1.260(18)$ & $1.314(6)$ & $1.240(41)$ \\
$\langle 1\rangle_{\Delta u^{+}}$ & $0.794(46)$ & $0.814(12)$ & $0.831(21)$ & $0.812(22)$ \\
$\langle 1\rangle_{\Delta d^{+}}$ & $-0.453(52)$ & $-0.456(11)$ & $-0.476(22)$ & $-0.428(31)$ \\
$\langle 1\rangle_{\Delta s^{+}}$ & $-0.120(81)$ & $-0.112(23)$ & $-0.109(20)$ & $-0.038(96)$ \\
$\langle x\rangle_{\Delta u^{-}-\Delta d^{-}}$ & $0.195(14)$ & $0.203(9)$ & - & $0.241(26)$ \\
\hline
\end{tabular}

Table 3.6: Status of current global-fit determinations of the benchmark moments of polarized PDFs listed in Sec. 3.1.1. All values are shown at $\mu^{2}=4 \mathrm{GeV}^{2}$.

- The central value of the strong coupling at the $Z$-boson mass, $\alpha_{s}\left(M_{Z}^{2}\right)$ should be fixed at an agreed common value, consistent with the PDG world-average [28] $\left(\alpha_{s}\left(M_{Z}\right)=0.118\right)$.

- All known experimental and procedural sources of uncertainty should be properly accounted for.

The ABMP2016 set (as well as its previous versions) does not meet the second and third criteria; the CJ15 set does not meet the first, second and fourth criteria, while the HERAPDF2.0 set does not meet the first criterion. Hence, these sets were not included in the PDF4LHC2015 PDF set, although the possibility of including them in future versions of the recommendation remains open.

In order not to loose important information contained in the PDF sets excluded from the PDF4LHC recommendations, we also provide alternative benchmark numbers. Specifically, we combined all the numbers quoted in Table 3.5 so that the mean value is an unweighted average of the mean values and the error is half of the difference between the smallest and the largest result. The rationale for this choice is that PDF sets entering the PDF4LHC recommendations are not benchmarked in the $x \gtrsim 0.1$ region, which can be relevant for the moment analysis. The combination of all results in Table 3.5, although sometimes less precise than the PDF4LHC combination, maximizes the amount of experimental information included in the benchmark numbers. Specifically, it includes the information taken into account at large $x$ and small $Q^{2}$ in the CJ15 and ABMP16 PDF sets, which is otherwise excluded from the PDF4LHC set.

The global-fit benchmark numbers for $\langle x\rangle_{u^{+}-d^{+}},\langle x\rangle_{u^{+}},\langle x\rangle_{d^{+}},\langle x\rangle_{s^{+}}$and $\langle x\rangle_{g}$ will be further commented below, where they will be collected together with their lattice-QCD counterparts in Table 3.7.

Polarized parton distributions. We summarize the current status of global-fit results of the benchmark moments of polarized PDFs listed in Sec. 3.1.1 in Table 3.6. In the first column, we indicate the computed moment, and in the subsequent three columns, its value as obtained from the most recent available PDF determinations: NNPDFpol1.1 [16], DSSV08 [17] ${ }^{10}$, JAM15 [18] and JAM17 [165]. The most relevant features of these PDF sets have been presented in Sec. 2.3.3. All values in Table 3.5 are displayed at $\mu^{2}=4 \mathrm{GeV}^{2}$ at NLO. The uncertainties correspond to $68 \%$-CL bands with tolerance of $\Delta \chi^{2}=1$ for the DSSV08 PDF set. In the case of the JAM15 set, we do not provide a value for $\langle x\rangle_{\Delta u^{-}-\Delta d^{-}}$: the fit is based on inclusive DIS data only, which are not sensitive to the valence distribution $\Delta u^{-}-\Delta d^{-}$. We emphasize that, because of extrapolation uncertainties difficult to quantify, the error estimates in Table 3.6 should be interpreted as a lower bound, especially for the DSSV08 and JAM sets based on conventional parametrizations. In these cases, uncertainty bands are naively extrapolated from the measured kinematic region, therefore they are likely to underestimate the contribution coming from the small- $x$ region.

\footnotetext{
${ }^{10}$ The DSSV08 analysis has been updated by the DSSV14 analysis [27] essentially only in the determination of the gluon PDF. The moments in Table 3.6 therefore hardly differ in the two analyses.
} 


\begin{tabular}{lccc}
\hline Moment & Lattice QCD & Global Fit (PDF4LHC) & Global fit (uw avg) \\
\hline$\langle x\rangle_{u^{+}-d^{+}}$ & $0.119-0.226$ & $0.155(5)$ & $0.161(18)$ \\
$\langle x\rangle_{u^{+}}$ & $0.453(75)^{\dagger}$ & $0.347(5)$ & $0.352(12)$ \\
$\langle x\rangle_{d^{+}}$ & $0.259(74)^{\dagger}$ & $0.193(6)$ & $0.192(6)$ \\
$\langle x\rangle_{s^{+}}$ & $0.092(41)^{\dagger}$ & $0.036(6)$ & $0.037(3)$ \\
$\langle x\rangle_{g}$ & $0.267(35)^{\dagger}$ & $0.414(9)$ & $0.411(8)$ \\
\hline
\end{tabular}

Table 3.7: Benchmark values for lattice-QCD calculations and global-fit determinations of the benchmark moments of unpolarized PDFs. All values are shown at $\mu^{2}=4 \mathrm{GeV}^{2}$. Results with a superscript $\dagger$ are from a single lattice calculation; they may underestimate some sources of uncertainty.

As outlined in Sec. 2.3.3, polarized PDFs cannot be determined in a global QCD analysis with the same accuracy as their unpolarized counterparts. Also, because polarized PDFs do not enter precision physics studies at the LHC, the selection and combination of different PDF sets has received much less attention. No recommendations analogous to those from the PDF4LHC working group exist for polarized PDFs.

To provide a benchmark value for the relevant moments of polarized PDFs listed in Table 3.6, we apply an unweighted average of the NNPDFpol1.1, DSSV08 and JAM15 PDF sets. The rationale for this choice is twofold. On the one hand, we maximize the amount of experimental information that can determine the central value of our benchmark moments. As explained in Sec. 2.3.3, the NNPDFpol1.1 and the DSSV08 PDF sets are based on a very similar set of inclusive DIS data, while the JAM15 PDF set is based on a much wider inclusive DIS data set. This wider set can help constrain the moments of the total quark distributions. The NNPDFpol1.1 and the DSSV08 PDF sets are based respectively on $p p$ and SIDIS data to disentangle the quark and antiquark distributions. This can help constrain the moments of the valence distributions. On the other hand, we balance the rather different uncertainties among the three PDF sets, specifically the larger NNPDFpol1.1 estimate against the smaller DSSV08 and JAM15 values. This way, we avoid a possible underestimation of the procedural uncertainties induced for example by the choice of a simple PDF parametrization in the DSSV08 and JAM15 fits, or by the extrapolation to the small- $x$ region. Because the JAM17 set is unique in fitting simultaneously polarized PDFs and FFs, we do not include it in our benchmark average, but quote it as a useful comparison.

The global-fit benchmark numbers for $g_{A},\langle 1\rangle_{\Delta u^{+}},\langle 1\rangle_{\Delta d^{+}},\langle 1\rangle_{\Delta s^{+}}$and $\langle x\rangle_{\Delta u^{-}-\Delta d^{-}}$will be further commented below, where they will be collected with their lattice-QCD counterparts in Table 3.8.

\subsection{Comparing lattice-QCD and global-fit benchmark moments}

We can now compare the lattice-QCD and global PDF fit results presented in Secs. 3.1.2-3.1.3 for the unpolarized and polarized PDF moments respectively.

Unpolarized parton distributions. The benchmark values of the first moments of the unpolarized PDFs, obtained as described in Secs. 3.1.2-3.1.3, are summarized in Table 3.7. Both the PDF4LHC and the unweighted average (uw avg) are displayed in the case of global fits. The results from a single lattice calculation, which might underestimate some sources of uncertainty, are denoted with a superscript $\dagger$. All values shown here are at $\mu^{2}=4 \mathrm{GeV}^{2}$. For ease of comparison, these benchmark results are also graphically compared in Fig. 3.2, both in terms of absolute values (left panel) and of uncorrelated ratios to the lattice central values (right panel). 

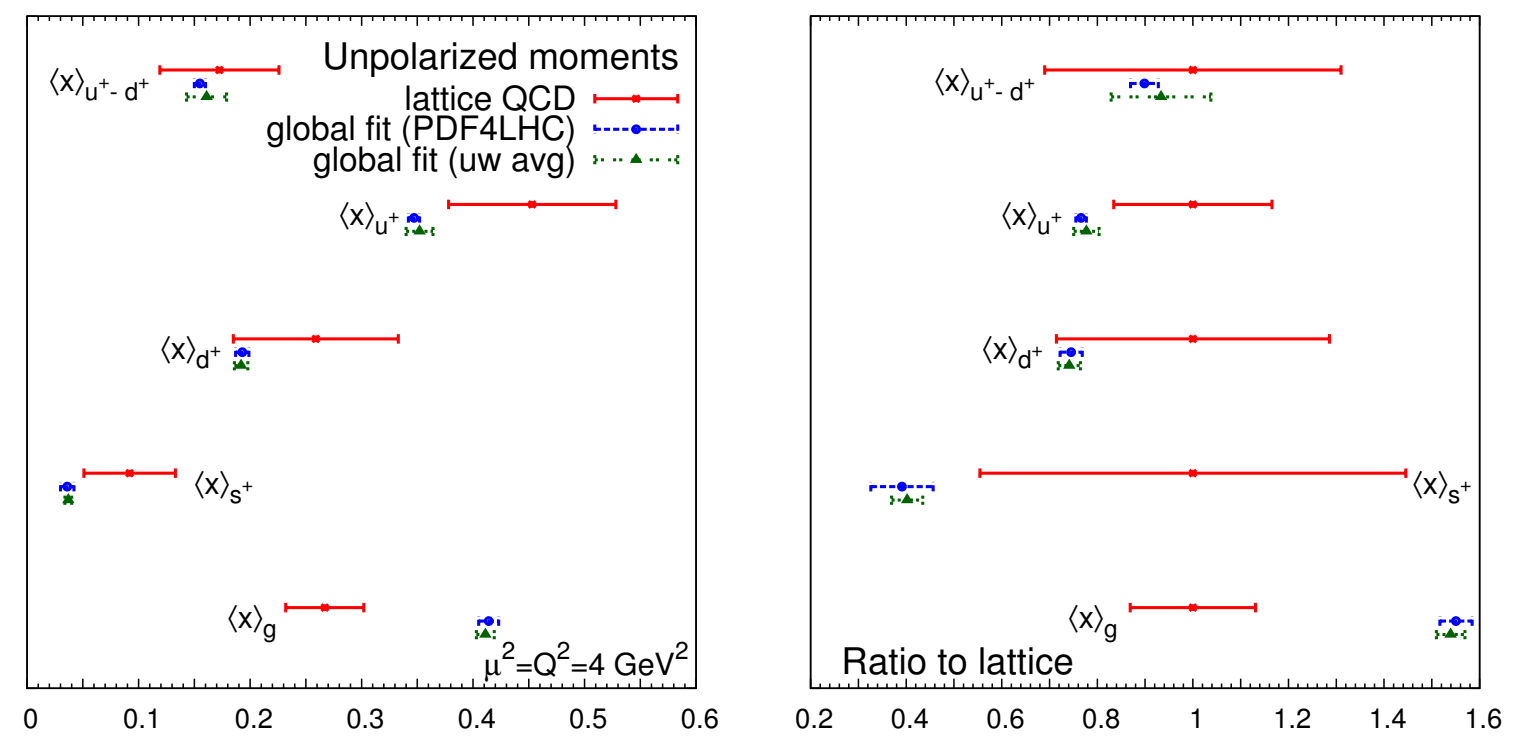

Figure 3.2: A comparison of the unpolarized PDF benchmark moments between the lattice QCD computations and global fit determinations. Results are displayed both in terms of absolute values (left) and ratios to the lattice values (right) at $\mu^{2}=4 \mathrm{GeV}^{2}$.

As is apparent from Table 3.7 and Fig. 3.2, there is a significant difference in the uncertainties between the lattice QCD and global fit results, with the latter always about one order of magnitude smaller than the former. Moreover, even within their large uncertainties, the lattice-QCD results for the first moments of the total up and strange quark and the gluon PDFs are not compatible with their global-fit counterparts. In the case of quarks, the discrepancy is below $2 \sigma$ (in units of the lattice-QCD uncertainty), while in the case of the gluon the discrepancy is slightly larger than $3 \sigma$.

On the lattice-QCD side, we note that in the flavor-singlet sector calculations neglected part of the renormalization and computed some other parts only perturbatively. Most of the discrepancies between lattice-QCD and global-fit results are observed in the flavor-singlet sector. Progress in taking into account the renormalization properly could shift lattice-QCD results significantly, and reconcile them with the global fits in the future. We also note that the momentum sum rule, Eq. (2.49), usually is not imposed in lattice-QCD calculations. In the ETMC 17 analysis [248], it turns out to be 1.071(93)(72), see Table 3.1, if uncertainties are assumed to be uncorrelated. Although there is no evidence for a violation of the momentum sum rule based on this result, one must be careful combining results from different calculations to account for correlations and other sources of error. Also, note that the ETMC 17 analysis is performed with $N_{f}=2$ flavors, hence the strange quark should not participate in the sum rule.

On the global-fit side, we note that the amount of experimental information that constrains the total up-quark distribution is the largest among all distributions. Therefore, it seems unlikely that its global-fit central value could vary significantly in the future, and become compatible with the current lattice result. Conversely, the amount of experimental information that constrains the total strange distribution in a global fit is less abundant and less accurate. A slight shift in its central value, towards the current lattice-QCD results, might be observed in the future, as soon as new data sensitive to the strange quark becomes available. Finally, in an attempt to reconcile the lattice-QCD and the global-fit results of the first moment of the gluon PDF, one could assume a completely different behavior of the gluon PDF below the HERA kinematic coverage, $x \sim 10^{-5}$ (see Fig. 2.2). While such a kinematic region remains completely unexplored, in general the contribution of this region to the moments is negligible and thus unlikely to resolve the situation. 


\begin{tabular}{lcc}
\hline Moment & Lattice QCD & Global Fit \\
\hline$g_{A} \equiv\langle 1\rangle_{\Delta u^{+}-\Delta d^{+}}$ & $1.195(39)\left(N_{f}=2+1+1\right)$ & $1.275(12)$ \\
$\langle 1\rangle_{\Delta u^{+}}$ & $0.830(26)^{\dagger}$ & $0.813(25)$ \\
$\langle 1\rangle_{\Delta d^{+}}$ & $-0.386(17)^{\dagger}$ & $-0.462(29)$ \\
$\langle 1\rangle_{\Delta s^{+}}$ & $-0.052--0.014$ & $-0.114(43)$ \\
$\langle x\rangle_{\Delta u^{-}-\Delta d^{-}}$ & $0.146-0.279$ & $0.199(16)$ \\
\hline
\end{tabular}

Table 3.8: Same as Table 3.7, but for the polarized benchmark moments.
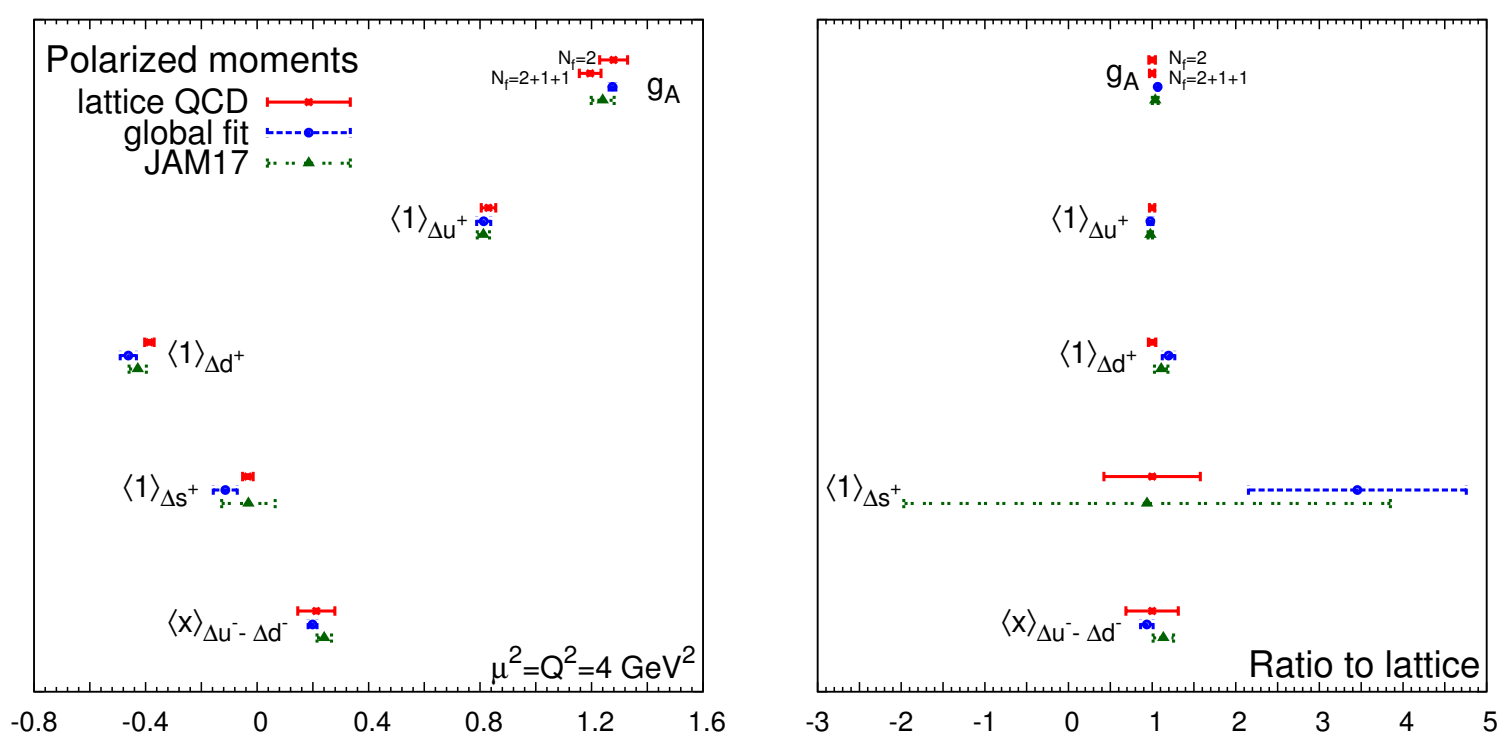

Figure 3.3: Same as Fig. 3.2, but for the polarized benchmark moments.

All these remarks apply irrespective of the benchmark value used for global fits, either the PDF4LHC or the unweighted average. They also still hold if individual lattice-QCD and/or global-fit results in Tables 3.1-3.5 are compared instead of their benchmark values in Table 3.7. These results suggest that both greater accuracy and greater precision are required in lattice-QCD calculations to match the accuracy and precision of the first moments of unpolarized PDFs determined from a global fit.

Polarized parton distributions. The benchmark values of the first moments of the unpolarized PDFs, obtained as described in Secs. 3.1.2-3.1.3, are summarized in Table 3.8. Results from a single lattice calculation, which might underestimate some sources of uncertainty, are denoted with a superscript $\dagger$. In the case of $g_{A}$, we report the two values with $N_{f}=2+1+1$ and $N_{f}=2$ sea quarks from lattice QCD. The value of $g_{A}$ is scale-independent, and we quote all other results at $\mu^{2}=4 \mathrm{GeV}^{2}$. For ease of comparison, these values are also displayed in Fig. 3.3 in the same format as in Fig. 3.2. In the case of $g_{A}$, the result with $N_{f}=2+1+1$ is used as normalization factor in the right panel of Fig. 3.3. Results from the JAM17 analysis [165], see Table 3.6, are displayed separately. The reason for this is that, in contrast with the NNPDFpol1.1, DSSV08 and JAM15 fits, in the JAM17 fit the experimental value of $g_{A}$, Eq. (2.50), is not an input of the fit, but it is fitted alongside the PDFs. Furthermore, in JAM17 PDFs are fitted alongside FFs.

As is apparent from Table 3.8 and Fig. 3.3, the size of the uncertainties on the moments is in general 
comparable between the lattice-QCD and the global-fit results, opposite to the unpolarized case ${ }^{11}$. The corresponding central values are also in reasonable agreement within their mutual uncertainties.

In the case of $g_{A}$, the global-fit result obtained from the unweighted average of the NNPDFpol1.1, DSSV08 and JAM15 fits shows a preference for the lattice-QCD result obtained with $N_{f}=2$ sea quarks (compared to that with $N_{f}=2+1+1$ sea quarks). Its uncertainty is, however, four times smaller than that of both lattice results. This is not unexpected, since, in all the three fits that are combined, the experimental value of $g_{A}$ is imposed in the fits themselves. The final uncertainty on the global-fit value of $g_{A}$ is thus reduced by the uncertainty of its experimental value $g_{A}^{\text {exp }}$, which is almost one order of magnitude smaller than the uncertainty on the lattice-QCD results (see Fig. 3.1). If the experimental value of $g_{A}$ is not imposed as a boundary condition in the fit, as in the JAM17 analysis, the size of the uncertainty on $g_{A}$ is comparable to that of the lattice results, although it is not able to discriminate between the $N_{f}=2$ or the $N_{f}=2=1+1$ results. Overall, this is a noteworthy confirmation of $\mathrm{SU}(2)$ symmetry in QCD to almost $2 \%$.

In the case of the zeroth moments of the total polarized quark distributions, the uncertainty on the lattice-QCD result is comparable to (in the case of $\langle 1\rangle_{\Delta u^{+}}$) or smaller than (in the case of $\langle 1\rangle_{\Delta d^{+}}$ and $\langle 1\rangle_{\Delta s^{+}}$) the uncertainty on the global-fit result. However, in the case of the zeroth moments of the total down- and strange-quark distributions, the lattice-QCD and the global-fit results are discrepant by about two $\sigma$ (in units of the lattice QCD uncertainty). On the one hand, we observe that the uncertainty on the lattice-QCD results might have been underestimated because of the lack of full control over all systematics (see Sec. 3.1.2). On the other hand, we observe that the global-fit result has been obtained by requiring $\mathrm{SU}(3)$ symmetry, i.e., by imposing in the individual fits the experimental value (with a possibly inflated uncertainty) of the octet PDF combination, as explained in Sec. 2.3.3. Relaxing this constraint can reconcile the discrepancy observed between the lattice-QCD and the global-fit result for the zeroth moments of the total down and strange PDFs. This is demonstrated by comparison with the JAM17 result, whose uncertainty band nicely includes both the lattice-QCD and the global-fit benchmark values.

In the case of the first moment of the valence distribution $\Delta u^{-}-\Delta d^{-}$, the lattice-QCD and the global-fit results are in excellent agreement, although the uncertainty of the former is five times larger than that of the latter.

All these remarks still hold if individual lattice-QCD and/or global-fit results in Tables 3.2-3.6 are compared instead of their benchmark values in Table 3.8. These results suggest that lattice-QCD calculations could provide a useful input to global fits of polarized PDFs, especially in limiting the extrapolation uncertainty into the completely unknown small- $x$ region. This will become more and more useful as full control over all sources of systematic uncertainties is achieved.

\section{Improving PDF fits with lattice-QCD calculations}

In this section, we provide an estimate of the potential impact of future lattice-QCD calculations in global unpolarized and polarized PDF fits. This study is carried out with two publicly available tools: the Bayesian reweighting method [231,263] applied to the NNPDF3.1 [11] and NNPDFpol1.1 [16] sets; and the Hessian profiling method [264] applied to HERAPDF2.0 set [20]. Both methods allow us to quantify the impact of new measurements (or of future measurements, if pseudo-data are used) on PDFs without repeating the global analysis. The main limitation of these methods is that they are maximally reliable if the amount of information carried in by the new (pseudo-)data is moderate in comparison to that already included in the fit.

\footnotetext{
${ }^{11}$ Note that the uncertainty of $\langle 1\rangle_{\Delta s^{+}}$in the right panel of Fig. 3.3 appears much larger than the uncertainty of other quark moments because of the normalization value, which is very close to zero.
} 
For simplicity, we limit our study to the impact of a subset of the moments that can be computed using lattice QCD, focusing on those that can be currently calculated with the highest precision. Therefore, we restrict ourselves to the benchmark moments discussed in Sec. 3. We also consider pseudo-data based on $x$-space lattice-QCD calculations from the quasi-PDF approach discussed in Sec. 2.2.3. As we show, particularly in the unpolarized case, the constraining power of direct $x$-space calculations is superior to that of PDF moments.

\subsection{Impact of lattice calculations of PDF moments}

We start by quantifying the constraining power of projected lattice-QCD calculations of PDF moments on both unpolarized and polarized global fits. We define the settings for our study and present our results following Bayesian reweighting and Hessian profiling, respectively.

\subsubsection{Analysis settings}

In the unpolarized case, we consider the first moments (momentum fractions) of $q^{+}=q+\bar{q}$ (with $q=u, d, s)$, of the gluon, and of the isoscalar combination $u^{+}-d^{+}$. In the polarized case, we consider the zeroth moments (spin fractions) of $\Delta q^{+}$(with $q=u, d, s$ ) and of the isoscalar combination $g_{A}=$ $\Delta u^{+}-\Delta d^{+}$, and the first moment of the $\Delta u^{-}-\Delta d^{-}$combination. Using the notation outlined in Appendix A, we have

$$
\begin{aligned}
\langle x\rangle_{u^{+}},\langle x\rangle_{d^{+}},\langle x\rangle_{s^{+}},\langle x\rangle_{g},\langle x\rangle_{u^{+}-d^{+}} & \text {for the unpolarized case } \\
\langle 1\rangle_{\Delta u^{+}},\langle 1\rangle_{\Delta d^{+}},\langle 1\rangle_{\Delta s^{+}},\langle x\rangle_{\Delta u^{-}-\Delta d^{-}},\langle 1\rangle_{\Delta u^{+}-\Delta d^{+}} & \text {for the polarized case }
\end{aligned}
$$

We look at three different scenarios, which we denote as Scenario A, B, and C, for the projected total systematic uncertainty associated with lattice-QCD calculations. Our choice for this uncertainty is denoted by $\delta_{L}^{(i)}$. It is summarized in Table 4.1 for each PDF moment $i$ in Eqs. (4.1)-(4.2) and for each scenario. Current uncertainties on lattice-QCD results (see Secs. 3.1.2-3.2) are also quoted for comparison. We emphasize that, while trying to be reasonably realistic, we do not associate a given scenario with a specific time scale for the calculation. Our results provide a guide to the potential constraining power of future lattice-QCD calculations of PDF moments once included in global analyses.

Our choice of uncertainties in Table 4.1 is rather different for the unpolarized and polarized cases. For the unpolarized case, Scenario A is based on values of $\delta_{L}^{(i)}$ rather smaller than the typical uncertainties that affect state-of-the-art lattice-QCD calculations, see Table 3.7. As expected from Fig. 3.2, and as we have explicitly verified, including lattice-QCD pseudo-data with uncertainties of similar size as those of Table 3.7 leaves unpolarized PDFs essentially unchanged. Significantly reduced uncertainties $\delta_{L}^{(i)}$ must be assumed to demonstrate any impact on global fits. We assume that $\delta_{L}^{(i)}$ is typically larger for $\langle x\rangle_{s^{+}}$and $\langle x\rangle_{u^{+}-d^{+}}$, compared to the other moments, in line with what is observed from Table 3.7. Scenarios $\mathrm{B}$ and $\mathrm{C}$ are rather optimistic, in that they require systematic uncertainties to decrease by roughly a factor of two and a factor of four with respect to Scenario A. For the polarized case, Scenario A assumes that the uncertainties $\delta_{L}^{(i)}$ are similar to current uncertainties in state-of-the-art lattice-QCD calculations, see Sec. 3, and Table 3.8.

We note that a total systematic error of $\delta_{L}^{(i)} \sim 1 \%$ is probably the best that one can achieve within a lattice-QCD calculation in the near future, since at that level several other effects, such as QED corrections, become relevant. These are much more difficult to deal with. For both the polarized and the unpolarized case, the generalization of these projections to other conceivable scenarios is straightforward and can be obtained from the authors upon request. 


\begin{tabular}{cccccc}
\hline Scenario & \multicolumn{5}{c}{$\delta_{L}^{(i)}$ for unpolarized moments } \\
& $\langle x\rangle_{u^{+}}$ & $\langle x\rangle_{d^{+}}$ & $\langle x\rangle_{s^{+}}$ & $\langle x\rangle_{g}$ & $\langle x\rangle_{u^{+}-d^{+}}$ \\
\hline Current & $\sim 16 \%$ & $\sim 30 \%$ & $\sim 45 \%$ & $\sim 13 \%$ & $\sim 60 \%$ \\
$\mathrm{~A}$ & $3 \%$ & $3 \%$ & $5 \%$ & $3 \%$ & $5 \%$ \\
$\mathrm{~B}$ & $2 \%$ & $2 \%$ & $4 \%$ & $2 \%$ & $4 \%$ \\
$\mathrm{C}$ & $1 \%$ & $1 \%$ & $3 \%$ & $1 \%$ & $3 \%$ \\
\hline \multicolumn{6}{c}{} \\
\hline Scenario & \multicolumn{6}{c}{$\delta_{L}^{(i)}$ for polarized moments } \\
\hline \multirow{2}{*}{ Current } & $\sim 3 \%$ & $\sim 5 \%$ & $\sim 70 \%$ & $\sim 65 \%$ & $\sim 3 \%$ \\
$\mathrm{~A}$ & $5 \%$ & $10 \%$ & $100 \%$ & $70 \%$ & $5 \%$ \\
$\mathrm{~B}$ & $3 \%$ & $5 \%$ & $50 \%$ & $30 \%$ & $3 \%$ \\
$\mathrm{C}$ & $1 \%$ & $2 \%$ & $20 \%$ & $15 \%$ & $1 \%$ \\
\hline
\end{tabular}

Table 4.1: The three scenarios assumed for the total percentage systematic uncertainty $\delta_{L}^{(i)}$ in future latticeQCD calculations. The unpolarized (upper table) and polarized (lower table) PDF moments included in the analysis are shown. Current systematic uncertainties in state-of-the-art lattice-QCD calculations are also displayed according to the benchmark exercise performed in Sec. 3 (see also Tables 3.7 and 3.8 for the unpolarized and polarized cases, respectively).

\subsubsection{Bayesian reweighting analysis}

To quantify the impact of future lattice-QCD calculations on global fits in each of the three scenarios in Table 4.1, we use a procedure based on Bayesian reweighting analysis. We briefly describe this procedure here, and refer to [231,263] for additional details.

- We first generate pseudo-data for the lattice-QCD calculations of $\langle x\rangle_{u^{+}},\langle x\rangle_{d^{+}},\langle x\rangle_{s^{+}},\langle x\rangle_{g}$, and $\langle x\rangle_{u^{+}-d^{+}}$(for the unpolarized case), and $\langle 1\rangle_{\Delta u^{+}},\langle 1\rangle_{\Delta d^{+}},\langle 1\rangle_{\Delta s^{+}},\langle x\rangle_{\Delta u^{-}-\Delta d^{-}}$, and $\langle 1\rangle_{\Delta u^{+}-\Delta d^{+}}$(for the polarized case). We denote generically these moments by $\mathcal{F}_{i}$.

- We construct the associated pseudo-data, denoted by $\mathcal{F}_{i}^{(\exp )}$, by taking the central values from the corresponding NNPDF fits, NNPDF3.1 NNLO for the unpolarized case and NNPDFpol1.1 NLO for the polarized case. That is, we assume for simplicity that the central value of such future lattice calculations would coincide with the current ones from the global fit. ${ }^{12}$ As discussed in Sec. 2.3.2, this corresponds to computing the mean over the Monte Carlo replica sample,

$$
\mathcal{F}_{i}^{(\text {exp })} \equiv \frac{1}{N_{\text {rep }}} \sum_{k=1}^{N_{\text {rep }}} \mathcal{F}_{i}^{(k)}, \quad i=1, \ldots, N_{\text {mom }}
$$

where $N_{\text {mom }}$ is the number of PDF moments that will be included in the reweighting; here $N_{\text {mom }}=$ 5 both for the unpolarized and polarized cases. To be consistent with the calculations in Sec. 3, the central values of the pseudo-data, Eq. (4.3), are also evaluated at $Q^{2}=4 \mathrm{GeV}^{2}$ (see Tables 3.5 and 3.6).

\footnotetext{
${ }^{12}$ The exercise can be repeated with the actual lattice-QCD central values. However, this requires some choices, such
} as to how to impose the momentum sum rule. This is beyond the scope of the present studies. 
- The uncertainty in the pseudo-data, denoted by $\delta \mathcal{F}_{i}^{(\exp )}$, is taken to be the value indicated in Table 4.1 for each of the three scenarios. Thus, the absolute uncertainty on the $i$-th moment is given by $\delta \mathcal{F}_{i}^{(\exp )}=\delta_{L}^{(i)} \mathcal{F}_{i}^{(\exp )}$.

- Using the pseudo-data (central values and total uncertainties) as defined above, we compute the Bayesian weights $\omega_{k}$. These weights quantify the agreement between each $k$-th replica in the input $\mathrm{PDF}$ set and the corresponding lattice pseudo-data. We compute the $\chi^{2}$ for each replica $k$ as

$$
\chi^{2(k)}=\sum_{i=1}^{N_{\text {mom }}} \frac{\left(\mathcal{F}_{i}^{(k)}-\mathcal{F}_{i}^{(\exp )}\right)^{2}}{\left(\delta \mathcal{F}_{i}^{(\exp )}\right)^{2}}, \quad k=1, \ldots, N_{\mathrm{rep}},
$$

assuming that there are no correlations between different $N_{\text {mom }}$ moments. This assumption in general might not be a good approximation, since most lattice-QCD systematic errors are correlated among different moments, and can be avoided, provided the full breakdown of systematic errors for each quantity is available.

Once the values of the $\chi^{2}$ have been evaluated, we compute the corresponding weights for each replica. The relation between the weights $w_{k}$ and the values of the $\chi^{2(k)}$ of each replica is $[231,263]$

$$
\omega_{k}=\frac{\left(\chi^{2(k)}\right)^{\left(N_{\mathrm{mom}}-1\right) / 2} \exp \left(-\chi^{2(k)} / 2\right)}{\sum_{k=1}^{N_{\text {rep }}}\left[\left(\chi^{2(k)}\right)^{\left(N_{\mathrm{mom}}-1\right) / 2} \exp \left(-\chi^{2(k)} / 2\right)\right]},
$$

where the denominator ensures that the weight admits a probabilistic interpretation, that is, $\sum_{k} w_{k}=1$. These weights represent a measure of the agreement of the individual replicas with the new pseudo-data. For instance, replicas which have associated values of the moments far from the pseudo-data (within uncertainties) will have a large $\chi^{2}$ and a very small weight, being thus effectively discarded.

- These weights are used to recompute the PDFs, their moments, and generic cross-sections. This procedure emulates the impact of adding lattice-QCD pseudo-data to a complete PDF fit. For instance, after reweighting, the mean value of the PDF moments is

$$
\mathcal{F}_{i}^{(\mathrm{rw})}=\sum_{k=1}^{N_{\mathrm{rep}}} \omega_{k} \mathcal{F}_{i}^{(k)}, \quad i=1, \ldots, N_{\mathrm{mom}},
$$

with a similar relation for the associated uncertainties.

One limitation of the reweighting procedure is that it is maximally reliable if the effective number of replicas $N_{\text {eff }}$ that survive the reweighting procedure (which is a measure of the amount of information left) is not too small. This effective number of replicas is quantified in terms of the Shannon entropy [231, 263]

$$
N_{\text {eff }} \equiv \exp \left[\frac{1}{N_{\text {rep }}} \sum_{k=1}^{N_{\text {rep }}} \omega_{k} \log \left(N_{\text {rep }} / \omega_{k}\right)\right] .
$$

Finding $N_{\text {eff }} \ll N_{\text {rep }}$ means that the pseudo-data have a large impact on the fit, potentially leading to a large reduction of the PDF uncertainties. If either the effective number of replicas becomes too small (say $N_{\text {eff }} \lesssim 25$ ), or the relative fraction is small (say, $N_{\text {eff }} / N_{\text {rep }} \lesssim 0.10$ ), then the results become unreliable, since they are affected by large statistical fluctuations.

Therefore, before considering the effects of the lattice-QCD pseudo-data at the PDF level, we need to ensure that the three scenarios defined in Table 4.1 still lead to values of $N_{\text {eff }}$ large enough for the 


\begin{tabular}{lcc}
\hline & NNPDF3.1 & NNPDFpol1.1 \\
\hline$N_{\text {rep }}$ original & 1000 & 100 \\
$N_{\text {eff Scenario A }}$ & 740 & 72 \\
$N_{\text {eff Scenario B }}$ & 750 & 59 \\
$N_{\text {eff }}$ Scenario C & 510 & 20 \\
\hline
\end{tabular}

Table 4.2: The effective number of replicas $N_{\text {eff }}$, Eq. (4.7), remaining after pseudo-data on the PDF moments are included in the global fit according to the scenarios outlined in Table 4.1. For completeness, we also indicate the original number of replicas $N_{\text {rep }}$ for the prior PDF sets, NNPDF3.1 and NNPDFpol1.1.

\begin{tabular}{lcccc}
\hline & Original & Scenario A & Scenario B & Scenario C \\
\hline$\langle x\rangle_{u^{+}}$ & $0.348 \pm 0.005$ & $0.349 \pm 0.004$ & $0.349 \pm 0.004$ & $0.349 \pm 0.003$ \\
$\langle x\rangle_{d^{+}}$ & $0.196 \pm 0.004$ & $0.196 \pm 0.004$ & $0.196 \pm 0.003$ & $0.196 \pm 0.002$ \\
$\langle x\rangle_{s^{+}}$ & $0.0393 \pm 0.0036$ & $0.0389 \pm 0.0030$ & $0.0389 \pm 0.0024$ & $0.0389 \pm 0.0014$ \\
$\langle x\rangle_{g}$ & $0.4097 \pm 0.0042$ & $0.4097 \pm 0.0043$ & $0.4097 \pm 0.0040$ & $0.4097 \pm 0.0029$ \\
$\langle x\rangle_{u^{+}-d^{+}}$ & $0.1522 \pm 0.0033$ & $0.1521 \pm 0.0037$ & $0.1521 \pm 0.0035$ & $0.1521 \pm 0.0029$ \\
\hline
\end{tabular}

Table 4.3: Values of the unpolarized PDF moments used as pseudo-data, as well as the corresponding results after the reweighting has been performed, for the three scenarios summarized in Table 4.1. The PDF uncertainties quoted correspond in all cases to $68 \%$ CL intervals.

reweighting procedure to be reliable. In Table 4.2 we indicate the effective number of replicas $N_{\text {eff }}$, Eq. (4.7), remaining when the pseudo-data are included in the global fit according to the scenarios in Table 4.1. For completeness, we also quote the original number of replicas $N_{\text {rep }}$ for the prior PDF sets, NNPDF3.1 and NNPDFpol1.1, respectively. As we can see, there is a marked decrease of $N_{\text {rep }}$ for the three scenarios, indicating that adding the PDF moments leads to non-trivial constraints on the global fit. For instance, in the most optimistic scenario, Scenario C, the effective number of replicas is around two (five) times smaller than the starting number of replicas in the unpolarized (polarized) case.

Impact on unpolarized global fits. We now discuss the results of applying the reweighting procedure to NNPDF3.1. In Table 4.3 we summarize the values of the unpolarized PDF moments used as pseudo-data $\mathcal{F}_{i}^{(\exp )}$, and the corresponding results after the reweighting has been performed, for the three scenarios summarized in Table 4.1; PDF uncertainties correspond to 68\%-CL intervals. We recall that, as explained above, the three scenarios exhibit uncertainties $\delta_{L}^{(i)}$ for the lattice-QCD pseudo-data rather smaller than those of current state-of-the-art calculations (see Table 3.7).

From Table 4.3 we see that a significant reduction in the uncertainties in the unpolarized PDF moments is challenging to achieve unless we assume the most aggressive scenarios. For instance, in Scenario C, which is about the best precision that can be achieved from lattice-QCD in the near future, the PDF uncertainties of the first moments (that is, the momentum fractions) for $u^{+}, d^{+}, s^{+}$and $g$ decrease by around $30 \%-60 \%$. The most marked decrease is for the strange momentum fraction, since this is affected by the largest PDF error in the prior fit. In contrast, the nonsinglet combination $\langle x\rangle_{u^{+}-d^{+}}$ is essentially unchanged in all three scenarios. Note that, in Table 4.3, the central values of the PDF moments are stable, since we assume that the central values of the pseudo-data correspond to those of the input PDFs. In a realistic situation, this is not necessarily the case and central values of the PDFs could also vary.

Further evidence that reducing uncertainties in unpolarized PDFs will be challenging is shown in 

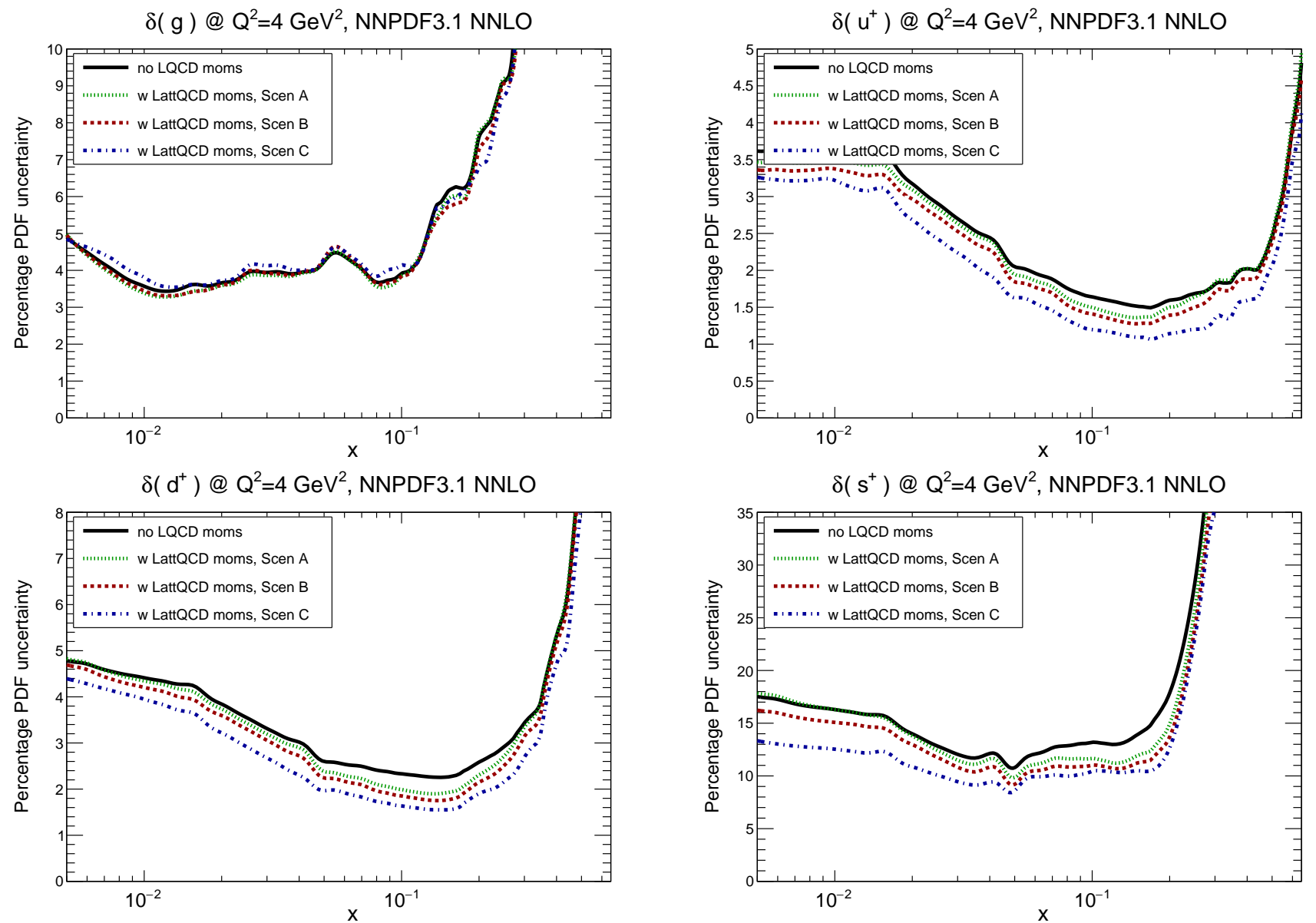

Figure 4.1: The percentage PDF uncertainty in NNPDF3.1 for the gluon and the $u^{+}, d^{+}$and $s^{+}$quark PDFs at $Q^{2}=4 \mathrm{GeV}^{2}$, compared to the results of including lattice-QCD pseudo-data for moments of PDFs in the fit, according to the three scenarios in Table 4.1. See text for details.

Fig. 4.1, which displays the percentage PDF uncertainties in NNPDF3.1 for the gluon and the $u^{+}, d^{+}$ and $s^{+}$quark PDFs at $Q^{2}=4 \mathrm{GeV}^{2}$, compared to the corresponding results including lattice-QCD pseudo-data. In the case of the $u^{+}, d^{+}$and $s^{+}$, we observe a slight reduction of the PDF uncertainties, which is more marked as we move from Scenarios A to C. For instance, in the latter case the percentage PDF uncertainty on $u^{+}\left(d^{+}\right.$and $\left.s^{+}\right)$at $x \simeq 0.1$ decreases from $1.8 \%$ to $1.2 \%$ (from $2.2 \%$ to $1.7 \%$ and from $13 \%$ to $10 \%$, respectively). The PDF uncertainties of the gluon PDF, however, are essentially unchanged even in the most optimistic scenario.

We also observe the trend that the reduction of the uncertainty of the PDF moments (see Table 4.3) is more significant than the PDF uncertainty as a function of $x$ (Fig. 4.1). We will see that this pattern also persists for the polarized PDF case. As the PDF moments integrate across all $x$ values (with emphasis on smaller $x$ values), this suggests that there are correlations which could be driving this result. In particular we note that in Scenario $\mathrm{C}$ the uncertainty on the moment for $s^{+}$is less than $4 \%$ while for the PDF at any $x$ it is always greater than $8 \%$, a result which can only be achieved due to strong anticorrelation between different $x$ regions. Additional studies examining the PDF correlations before and after inclusion of the lattice-QCD input could prove enlightening.

Focusing on the large- $x$ region, where the impact of the PDF moments considered here is expected to be more marked, in Fig. 4.2 we show the ratio of the uncertainty in each scenario to the prior PDF uncertainty in the NNPDF3.1 set, for the $d^{+}$and $s^{+}$total quark PDFs. This comparison clearly illustrates that the relative reduction of the PDF uncertainties upon addition of lattice-QCD pseudodata is not completely flat, and that it exhibits some structure. The constraints from lattice-QCD 

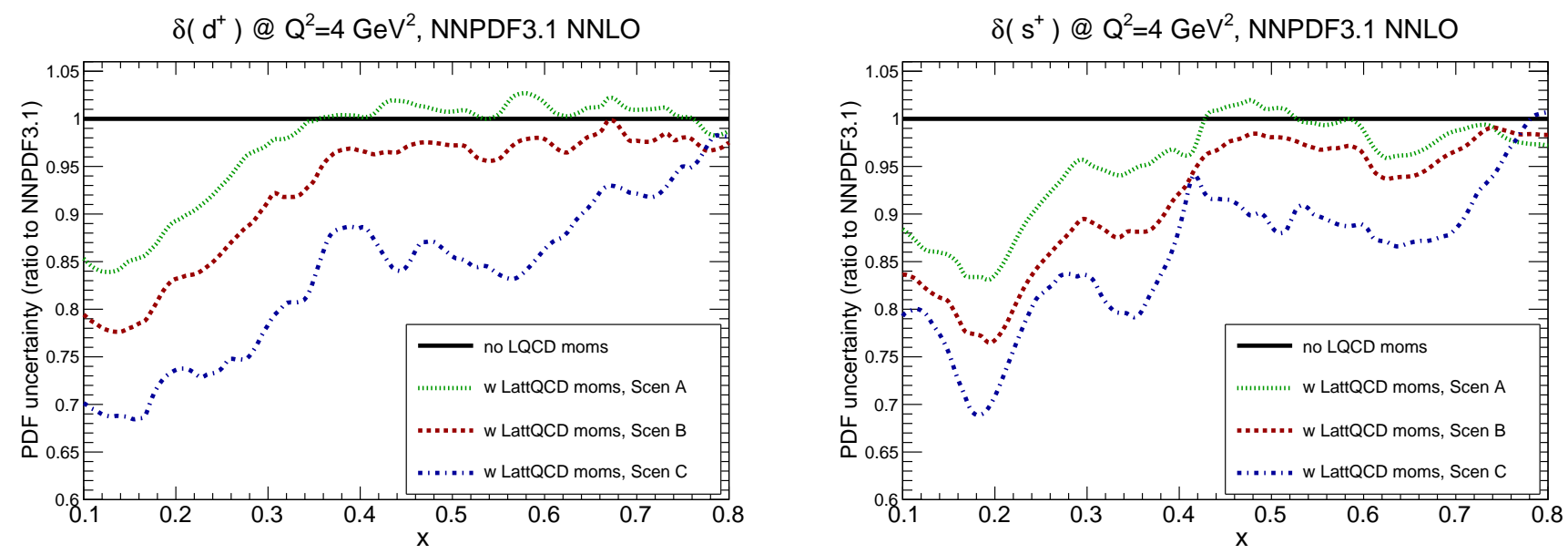

Figure 4.2: Same as Fig. 4.1, now focusing on the large- $x$ region, and showing the ratio of the PDF uncertainty in the fits based on the three scenarios to the original PDF uncertainty in the NNPDF3.1 set, for the $d^{+}$(left) and $s^{+}$(right) total quark PDFs.

\begin{tabular}{lcccc}
\hline & Original & Scenario A & Scenario B & Scenario C \\
\hline$\langle 1\rangle_{\Delta u^{+}}$ & $+0.788 \pm 0.079$ & $+0.798 \pm 0.039$ & $+0.797 \pm 0.023$ & $+0.790 \pm 0.009$ \\
$\langle 1\rangle_{\Delta d^{+}}$ & $-0.450 \pm 0.083$ & $-0.450 \pm 0.042$ & $-0.456 \pm 0.026$ & $-0.465 \pm 0.012$ \\
$\langle 1\rangle_{\Delta s^{+}}$ & $-0.124 \pm 0.108$ & $-0.120 \pm 0.070$ & $-0.121 \pm 0.076$ & $-0.111 \pm 0.029$ \\
$\langle 1\rangle_{\Delta u^{+}-\Delta d^{+}}$ & $+1.250 \pm 0.024$ & $+1.250 \pm 0.022$ & $+1.253 \pm 0.016$ & $+1.256 \pm 0.012$ \\
$\langle x\rangle_{\Delta u^{-}-\Delta d^{-}}$ & $+0.196 \pm 0.014$ & $+0.195 \pm 0.014$ & $+0.196 \pm 0.016$ & $+0.198 \pm 0.012$ \\
\hline
\end{tabular}

Table 4.4: Same as Table 4.3, now for the polarized PDF moments computed with NNPDFpol1.1. The corresponding impact at the PDF level is shown in Fig. 4.3.

calculations of these PDF moments decrease for larger values of $x$.

Impact on polarized global fits. Now we turn to apply the reweighting procedure to NNPDFpol1.1. In Table 4.4 we list the values of the polarized PDF moments used as pseudo-data, and the corresponding results after the reweighting has been performed for the three scenarios summarized in in Table 4.1. As in the unpolarized case, the PDF uncertainties quoted correspond in all cases to 68\%-CL intervals. As we can see from this comparison, in Scenario A (which assumes lattice-QCD pseudo-data with uncertainties similar to existing calculations) there is a marked impact on the polarized PDF moments. For both $\langle 1\rangle_{\Delta u^{+}}$and $\langle 1\rangle_{\Delta d^{+}}$the PDF uncertainties are roughly halved, with a similar, but less marked, trend for $\langle 1\rangle_{\Delta s^{+}}$. At this level, there is no impact on the nonsinglet combinations $\langle 1\rangle_{\Delta u^{+}-\Delta d^{+}}$and $\langle x\rangle_{\Delta u^{-}-\Delta d^{-}}$.

As we further decrease the assumed uncertainties in the lattice-QCD pseudo-data, we observe a corresponding reduction of the uncertainties in the global fit. In Scenario C, the most optimistic, we find that for both $\langle 1\rangle_{\Delta u^{+}}$and $\langle 1\rangle_{\Delta d^{+}}$there is an uncertainty reduction by about an order of magnitude compared to the current values, and by about a factor of five for $\langle 1\rangle_{\Delta s^{+}}$. Therefore, future lattice-QCD calculations of polarized PDF moments can potentially lead to a much more precise understanding of the spin structure of the proton. The other quark combinations exhibit less sensitivity to the inclusion of the PDF moments in the global fit, because they are already quite well constrained by available experimental data. The PDF uncertainties for $\langle 1\rangle_{\Delta u^{+}-\Delta d^{+}}$are reduced by a factor of two in this quite optimistic scenario, while those of $\langle x\rangle_{\Delta u^{-}-\Delta d^{-}}$are essentially unaffected even in the most optimistic 

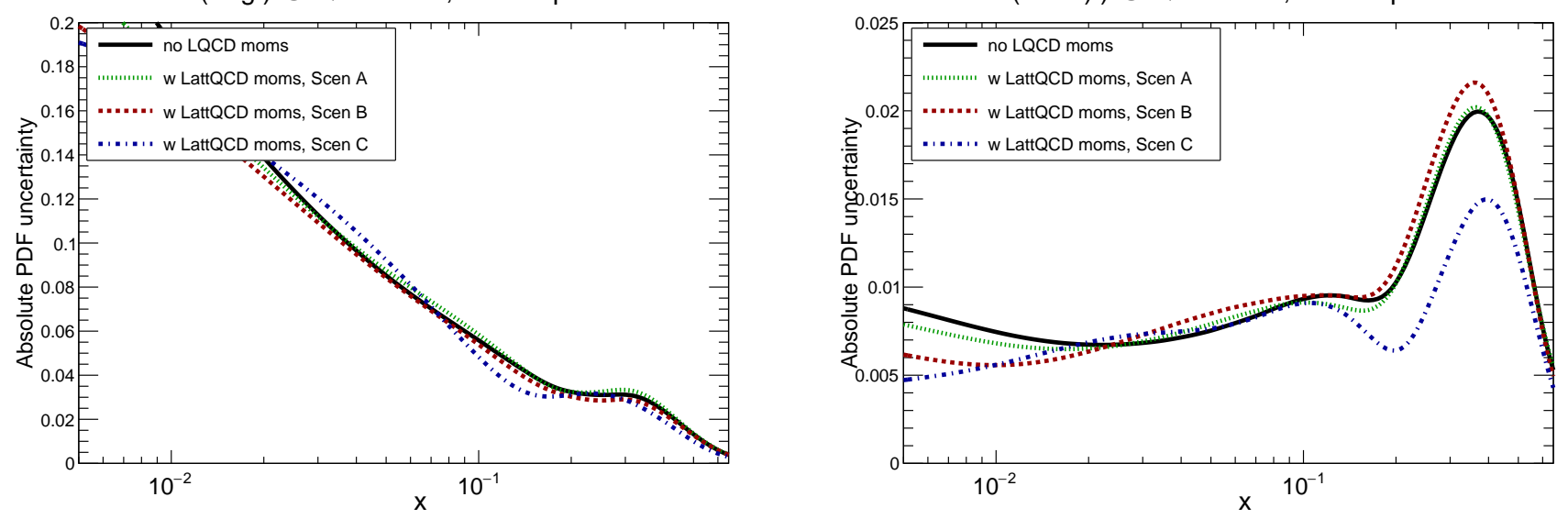

$\delta\left(\Delta \mathrm{d}^{+}\right) @ \mathrm{Q}^{2}=4 \mathrm{GeV}^{2}$, NNPDFpol1.1
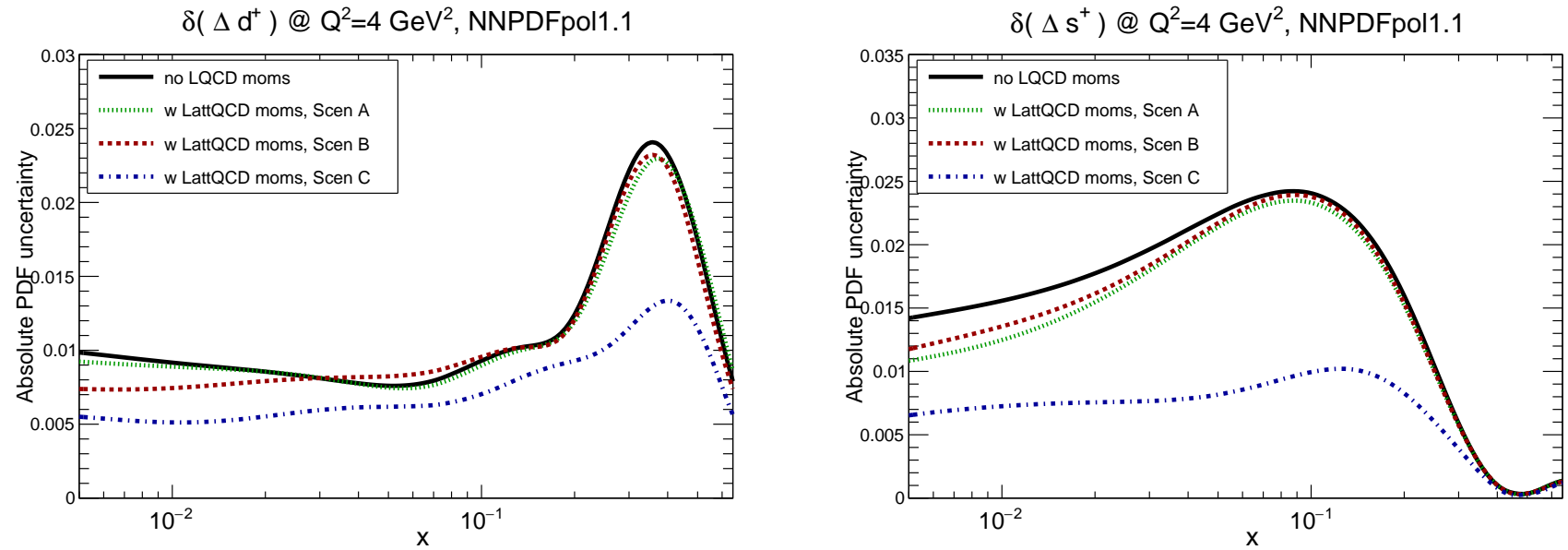

Figure 4.3: Same as Fig. 4.1, now showing the absolute PDF uncertainties of the NNPDFpol1.1 fit $Q^{2}=$ $4 \mathrm{GeV}^{2}$, compared to the corresponding results once the lattice pseudo-data on polarized moments in included in the analysis via reweighting.

scenario.

In Fig. 4.3 we compare the absolute PDF uncertainties of the NNPDFpol1.1 fit to the corresponding results once the lattice pseudo-data on polarized moments are included in the analysis by means of the reweighting. We show absolute rather than relative uncertainties because, unlike unpolarized PDFs, polarized PDFs often exhibit nodes (in particular for strangeness and the gluon) and in the nearby regions the concept of relative uncertainty becomes ill-defined.

From Fig. 4.3 we see that for scenarios A and B there is only a very moderate reduction (or even a slight increase) of the PDF uncertainties, seemingly at odds with the results for their moments in Table 4.4. The reason is that the first PDF moments alone provide only limited information on the shape of the PDFs themselves, and therefore in some cases one finds a larger error reduction on the moments (since these are the fitted quantities) than on the PDFs themselves (which are only indirectly constrained). Once, however, the lattice-QCD pseudo-data uncertainties decrease beyond a certain level, these uncertainties start to influence the PDF shape, as we can see from the results of Scenario C. In that case we find that the PDF uncertainties can decrease by up to a factor of two (three) for $\Delta d^{+}(x, Q)\left(\Delta s^{+}(x, Q)\right)$. We also see the apparently simple feature that relative reduction of PDF uncertainties is more or less constant along the whole range of $x$. For the strange quark this is perhaps roughly consistent with a simple reduction in the normalization uncertainty independent of $x$. However, similarly to the unpolarized case, for the down quark this decrease is a much smaller factor than the decrease in the uncertainty of the moments, meaning that there must be some anticorrelation between PDFs at different $x$ values. 


\subsubsection{Hessian profiling analysis}

To complement the results obtained with the Bayesian reweighting approach, we use a profiling method, suitable for Hessian PDF sets, to estimate the effect of including lattice-QCD pseudo-data into the fit [264, 265]. We choose HERAPDF2.0 [20] as a representative set of Hessian PDFs. As in the case of the Bayesian reweighting exercise presented in the previous section We consistently use the same lattice-QCD pseudo-data on PDF moments to estimate the impact on HERAPDF2.0. An additional advantage of the HERAPDF2.0 set is the use of a standard tolerance $\Delta \chi^{2}=1$ for defining the $68 \%$-CL PDF uncertainties, which enables a robust framework for applying the profiling method.

For Hessian PDF sets, the Hessian profiling method can be used to both check the compatibility of new data with a given PDF set, and also estimate the impact these data will have on the PDFs. In the following we describe the essential components of the profiling method, and assume that the Hessian PDF set uses a tolerance of $\Delta \chi^{2}=1$, which corresponds to $68 \%$ CL uncertainties, as is the case with the HERAPDF2.0 set. ${ }^{13}$ The central values of the considered moments are obtained using the central PDFs and the corresponding errors are calculated according to:

$$
\delta \mathcal{F}_{i}=\frac{1}{2} \sqrt{\sum_{k}\left(\mathcal{F}_{i}\left(f_{k}^{+}\right)-\mathcal{F}_{i}\left(f_{k}^{-}\right)\right)^{2}}, \quad i=1, \ldots, N_{\text {mom }},
$$

where $k$ labels the number of error PDFs (Hessian eigenvectors) which have both a positive and negative direction. In the profiling method, one considers a $\chi^{2}$ function in which the $\chi^{2}$ of the new data has been added to the initial $\chi_{0}^{2}$, namely

$$
\chi_{\text {new }}^{2}=\chi_{0}^{2}+\sum_{k}^{N_{\text {eig }}} z_{k}^{2}+\sum_{i=1}^{N_{\text {data }}} \frac{\left(\mathcal{F}_{i}-\mathcal{F}_{i}^{(\exp )}\right)^{2}}{\left(\delta \mathcal{F}_{i}^{(\exp )}\right)^{2}},
$$

where $\chi_{0}^{2}$ is the value of the $\chi^{2}$ function in the minimum of the initial PDF set, $z_{k}$ are the parameters diagonalizing the Hessian matrix of the initial PDF set, $N_{\text {eig }}$ is the dimension of the eigenvector space in which initial Hessian errors are defined (half of the number of error PDFs), $\mathcal{F}_{i}^{(\exp )}$ is the new (pseudo-)data, and $\mathcal{F}_{i}$ the corresponding theory prediction.

In the spirit of the Hessian method, the new theory predictions $\mathcal{F}_{i}$ can be expanded using a linear approximation:

$$
\mathcal{F}_{i} \simeq \mathcal{F}_{i}\left[S_{0}\right]+\left.\sum_{k} \frac{\partial \mathcal{F}_{i}[S]}{\partial z_{k}}\right|_{S=S_{0}} z_{k} \quad \simeq \mathcal{F}_{i}\left[S_{0}\right]+\sum_{k} D_{i k} w_{k}
$$

where $S_{0}$ represents the central PDF and we have defined

$$
D_{i k}=\frac{1}{2}\left(\mathcal{F}_{i}\left[S_{k}^{+}\right]-\mathcal{F}_{i}\left[S_{k}^{-}\right]\right) ;
$$

here the derivative has been approximated by a finite difference of the Hessian PDF error sets $S_{k}^{ \pm}$. The new $\chi^{2}$ of Eq. (4.9) can now be minimized with respect to the parameters $w_{k}$, which results in:

$$
w_{k}^{\min }=\sum_{n}-B_{k n}^{-1} a_{n}
$$

where we have introduced

$$
B_{k n}=\sum_{i} \frac{D_{i k} D_{i n}}{\left(\delta \mathcal{F}_{i}^{(\exp )}\right)^{2}}+\delta_{k n}, \quad a_{k}=\sum_{i} \frac{D_{i k}\left(\mathcal{F}_{i}\left[S_{0}\right]-\mathcal{F}_{i}^{(\exp )}\right)}{\left(\delta \mathcal{F}_{i}^{(\exp )}\right)^{2}} .
$$

\footnotetext{
${ }^{13}$ In this exercise we consider only the experimental HERAPDF2.0 uncertainties, but not the model and parametrization variations, which are not suited for profiling.
} 


\begin{tabular}{lcccc}
\hline & Original & Scenario A & Scenario B & Scenario C \\
\hline$\langle x\rangle_{u^{+}}$ & $0.3720 \pm 0.0036$ & $0.3720 \pm 0.0030$ & $0.3720 \pm 0.0027$ & $0.3720 \pm 0.0020$ \\
$\langle x\rangle_{d^{+}}$ & $0.1845 \pm 0.0053$ & $0.1845 \pm 0.0028$ & $0.1845 \pm 0.0023$ & $0.1845 \pm 0.0015$ \\
$\langle x\rangle_{s^{+}}$ & $0.0346 \pm 0.0037$ & $0.0346 \pm 0.0015$ & $0.0346 \pm 0.0012$ & $0.0346 \pm 0.0009$ \\
$\langle x\rangle_{g}$ & $0.4006 \pm 0.0078$ & $0.4006 \pm 0.0042$ & $0.4006 \pm 0.0035$ & $0.4006 \pm 0.0024$ \\
$\langle x\rangle_{u^{+}-d^{+}}$ & $0.1875 \pm 0.0074$ & $0.1875 \pm 0.0045$ & $0.1875 \pm 0.0039$ & $0.1875 \pm 0.0027$ \\
\hline
\end{tabular}

Table 4.5: Values of the unpolarized PDF moments used as lattice-QCD pseudo-data, as well as the corresponding results after the profiling for the three scenarios summarized in Table 4.1. The HERAPDF2.0 PDFs were used, and the PDF uncertainties quoted correspond in all cases to 68\% CL intervals. The corresponding results of applying the reweighting method to NNPDF3.1 were listed in Table 4.3.

The key result of the Hessian profiling method is that now the components of the solution $w_{k}^{\text {min }}$ define a new set of PDFs representing a global minimum after including the new data:

$$
f_{\text {new }}=f_{S_{0}}+\sum_{k=1}^{N_{\text {eig }}} \frac{f_{S_{k}^{+}}-f_{S_{k}^{-}}}{2} w_{k}^{\min } .
$$

A set of new error PDFs can be also defined; in this case the matrix $B_{k n}$ plays the role of the Hessian matrix from which the PDF uncertainties can be obtained.

We performed this study using the xFitter program [266] assuming the same three scenarios for the lattice-QCD pseudo-data as in Table 4.1. The results are shown in Table 4.5, where we tabulate the uncertainties of the input HERAPDF2.0 PDF in column two and the corresponding uncertainties for each scenario in columns three to five. The analogous results from the reweighting method, applied to the NNPDF3.1 data set, were listed in Table 4.3.

From a comparison of the constraining power of the lattice-QCD pseudo-data displayed in Table 4.5 to Table 4.3, we observe a consistent trend between Bayesian reweighting of NNDPF3.1 and Hessian profiling of HERAPDF2.0. The PDF uncertainties for $\langle x\rangle_{d^{+}}\left(\langle x\rangle_{s^{+}}\right.$and $\left.\langle x\rangle_{g}\right)$ reduce by a factor of roughly four (four and three, respectively) compared to the original HERAPDF2.0 uncertainties. When comparing with Sec. 4.1.2, the initial uncertainties of the HERAPDF2.0 analysis are affected by the choice of data (DIS data only), and the number and form of the parameterization (14 parameter HERAPDF form); the final uncertainties are determined by the profiling procedure. In particular the profiling for the HERAPDF2.0 study assigns an effective uncertainty on the pseudodata corresponding to $\Delta \chi^{2}=1$, whereas the constraint in the NNPDF study is weaker, as it would be for a PDF set with eigenvectors, but which applied a tolerance criterion. While these initial studies are instructive, further comparisons of these analyses would be valuable.

In Fig. 4.4 we present a comparison of the $u^{+}, d^{+}, g$, and $s^{+}$PDFs at the scale of $Q^{2}=4 \mathrm{GeV}^{2}$ between the original HERAPDF2.0 set and the results of the profiling exercise for Scenarios A, B and C. Only the experimental PDF uncertainties are shown in this comparison, but not the model and parametrization variations. The corresponding results based on the reweighting of NNPDF3.1 were shown in Figs. 4.1 and 4.2.

From Fig. 4.4 we see that, as expected, the impact of the lattice pseudo-data is greatest in the medium and large- $x$ regions. The precise impact on the PDFs is rather similar for the three scenarios, with the most optimistic Scenario C leading to the largest reduction in uncertainties. The quark flavor combinations that are most affected by the lattice-QCD pseudo-data are the $d^{+}$and $s^{+}$PDFs, and, to a lesser extent, the gluon PDF. The improvement in the PDF uncertainties for $d^{+}$and $s^{+}$occurs because the DIS data used in HERAPDF2.0 include only limited constraints on quark flavor separation, and, for these PDFs, the lattice-QCD pseudo-data add important new information. 

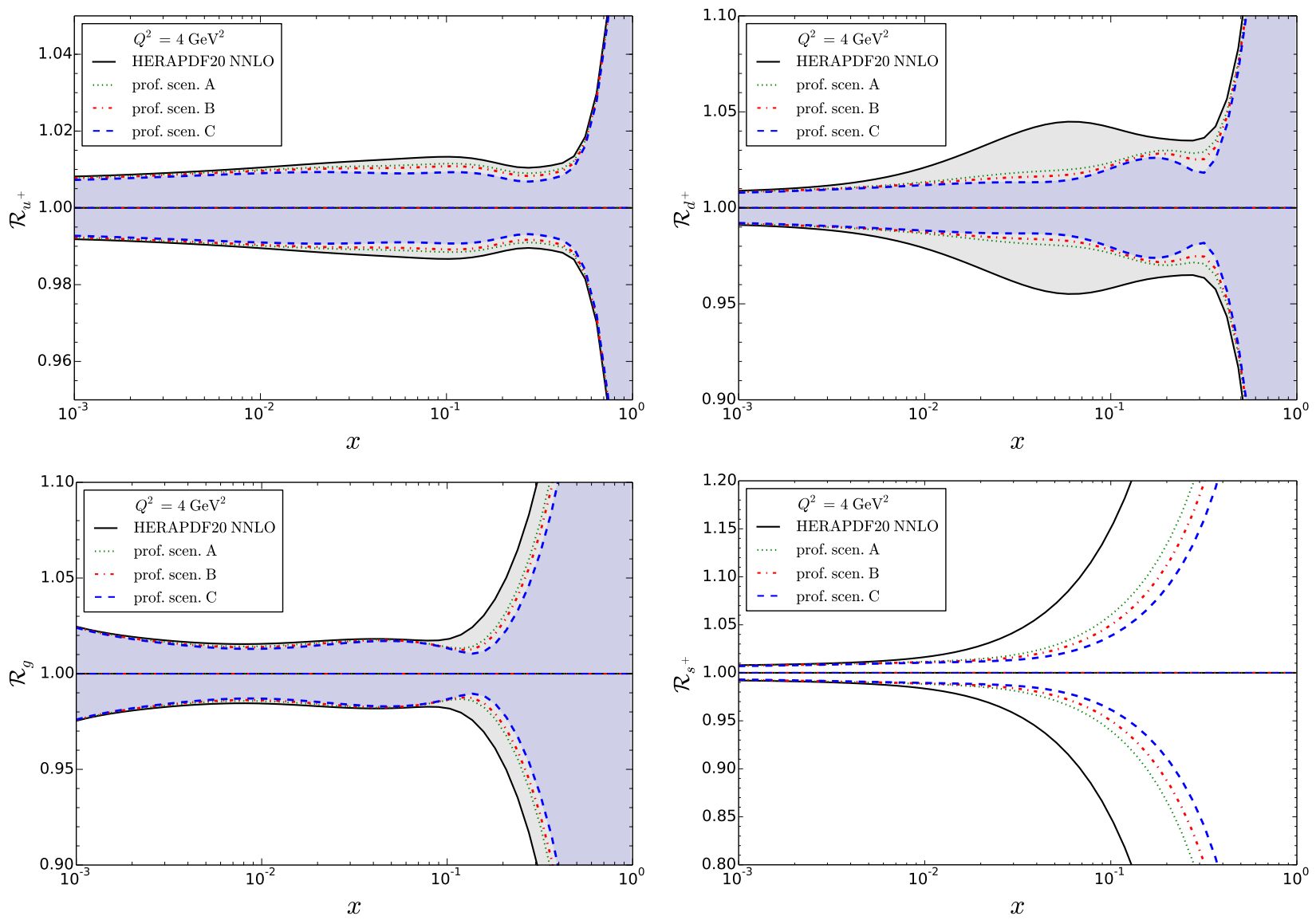

Figure 4.4: Comparison of the $u^{+}, d^{+}, g$, and $s^{+}$PDFs at the scale of $Q^{2}=4 \mathrm{GeV}^{2}$ between the original HERAPDF2.0 set and the results of the profiling exercise accounting for the constraints of the lattice-QCD moments pseudo-data in Scenarios A, B and C. Only the experimental PDF uncertainties are shown, but not the model and parametrization variations.

\subsection{Impact of lattice calculations of $x$-space PDFs}

In the previous section, we studied the impact of lattice-QCD calculations of PDF moments. We now perform an exploration of the potential constraints that future lattice QCD calculations of $x$-space PDFs can provide on global analyses. We focus on the isotriplet combination $x u-x d$ (and $x \Delta u-x \Delta d$ in the polarized case), the quark combination on which initial studies have been focused, as it is the simplest to calculate, owing to the lack of disconnected diagrams and the absence of mixing with other quark flavors or with gluons.

Following the same Bayesian reweighting procedure employed for PDF moments in Sec. 4.1.2, we have generated pseudo-data for the isotriplet combinations

$$
u\left(x_{i}, Q^{2}\right)-d\left(x_{i}, Q^{2}\right) \quad \text { and } \quad \bar{u}\left(x_{i}, Q^{2}\right)-\bar{d}\left(x_{i}, Q^{2}\right), \quad i=1, \ldots, N_{x},
$$

for the unpolarized case, and for

$$
\Delta u\left(x_{i}, Q^{2}\right)-\Delta d\left(x_{i}, Q^{2}\right) \quad \text { and } \quad \Delta \bar{u}\left(x_{i}, Q^{2}\right)-\Delta \bar{d}\left(x_{i}, Q^{2}\right), \quad i=1, \ldots, N_{x}
$$

for the polarized case, with $N_{x}$ being the number of points in $x$-space that are being sampled. We take $Q^{2}=4 \mathrm{GeV}^{2}$, consistent with our choices for the exercise performed in Secs. 4.1.2-4.1.3.

We consider three scenarios, denoted by Scenario D, E, and F, for the total uncertainty $\delta_{L}^{(i)}$ that will be assigned to the lattice-QCD calculations of the specific quark combinations listed in Eqs. (4.15) and 


\begin{tabular}{lcc}
\hline & NNPDF3.1 & NNPDFpol1.1 \\
\hline$N_{\text {rep }}$ original & 1000 & 100 \\
$N_{\text {eff Scenario D }}$ & 376 & 41 \\
$N_{\text {eff Scenario E }}$ & 173 & 35 \\
$N_{\text {eff }}$ Scenario F & 64 & 22 \\
\hline
\end{tabular}

Table 4.6: The effective number of replicas $N_{\text {eff }}$, Eq. (4.7), remaining when the pseudo-data on the lattice-QCD calculations of Eqns. (4.15) and (4.16) are included in the global unpolarized and polarized fits.

(4.16). Lattice-QCD computations are expected to have the smallest systematic uncertainties at large $x$, so we choose the $N_{x}=5$ points to be

$$
x_{i}=0.70,0.75,0.80,0.85,0.90 .
$$

For each scenario, we assume the same relative error for each value of $\left\{x_{i}\right\}$, and we neglect possible correlations between neighboring $x$-points. We assume uncertainties of $\delta_{L}^{(i)}=12 \%, 6 \%$ and $3 \%$ for scenarios D, E, and F, respectively. Note that we assume the same values of $\delta_{L}^{(i)}$ for the polarized and unpolarized cases, as well as for both the quark and the antiquark isotriplet combinations Eqs. (4.15) and (4.16).

We summarize the results of this exercise in Fig. 4.5, where we plot the ratio of the PDF uncertainties in each Scenario A, B and C (D, E and F) to the uncertainty of the original NNPDF3.1 (NNPDFpol1.1) set. We show the impact on the PDF uncertainties in $\bar{u}$ and $\bar{d}$ at large- $x$ in the upper plots, with the corresponding comparison for $\Delta \bar{u}$ and $\Delta \bar{d}$ in the lower plots. We concentrate on the results for the individual quark flavors, even though the constraints are imposed on differences between flavors, as the former are of the more direct interest for phenomenology. From this comparison, we find that lattice-QCD calculations of the $x$-dependence of PDFs can significantly reduce the uncertainties for both unpolarized and polarized antiquarks in the large- $x$ region. Taking into account that the PDF uncertainties on the large- $x$ antiquarks are rather large, and that they enter a number of important Beyond the Standard Model (BSM) search channels (such as for instance for production of new heavy gauge bosons $W^{\prime}$ and $Z^{\prime}$ ), our analysis demonstrates that such calculations would have direct phenomenological implications. We note however that the curves in Fig. 4.5 fluctuate by a rather large amount. This might be due to the fact that the uncertainties of the original PDFs fluctuate, particularly at low scales.

Fig. 4.5 shows that in the unpolarized case the large- $x$ PDF uncertainties could be reduced to $60 \%$ of their original value. We also find that there are no large differences between the three scenarios, probably because the constraint is on quark differences not on individual flavors, so there is freedom for $\bar{u}$ and $\bar{d}$ to vary in a correlated fashion while still satisfying the constraint. However, it does suggest that a direct lattice-QCD calculation of $x \bar{u}-x \bar{d}$ does not need to reach uncertainties at the few-percent level to influence global fits. For the polarized PDFs, Fig. 4.5 demonstrates that the reduction in PDF uncertainties could be significantly more marked. For instance, in the case of $\Delta \bar{d}$, at $x \simeq 0.8$ the resulting PDF uncertainty from Scenario $\mathrm{F}$ is less than $50 \%$ of the original uncertainty.

In Table 4.6 we indicate the effective number of replicas $N_{\text {eff }}$, Eq. (4.7), remaining when the latticeQCD pseudo-data for Eqns. (4.15) and (4.16) are included in the global unpolarized and polarized fits. Here we find a marked decrease in $N_{\text {rep }}$ for the three scenarios, in particular for the unpolarized case. For example, in the most optimistic Scenario F, only 64 effective replicas remain out of the original sample of $N_{\text {rep }}=1000$ replicas. See Table 4.2 for the corresponding information at the level of PDF moments.

We emphasize that the results of this exercise must be interpreted with some care. First of all, the results depend sensitively on the specific values of $\left\{x_{i}\right\}$ that we have assumed for the lattice-QCD 

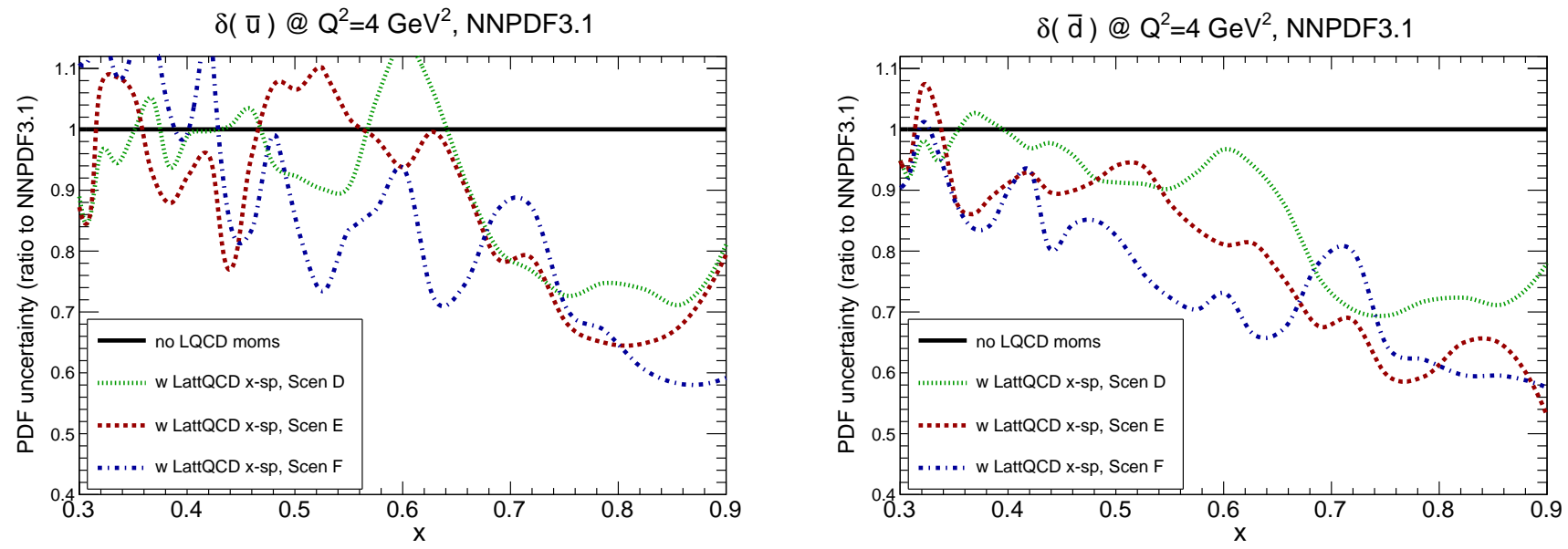

$\delta(\Delta \overline{\mathrm{u}}) @ \mathrm{Q}^{2}=4 \mathrm{GeV}^{2}, \mathrm{NNPDFpol1.1}$
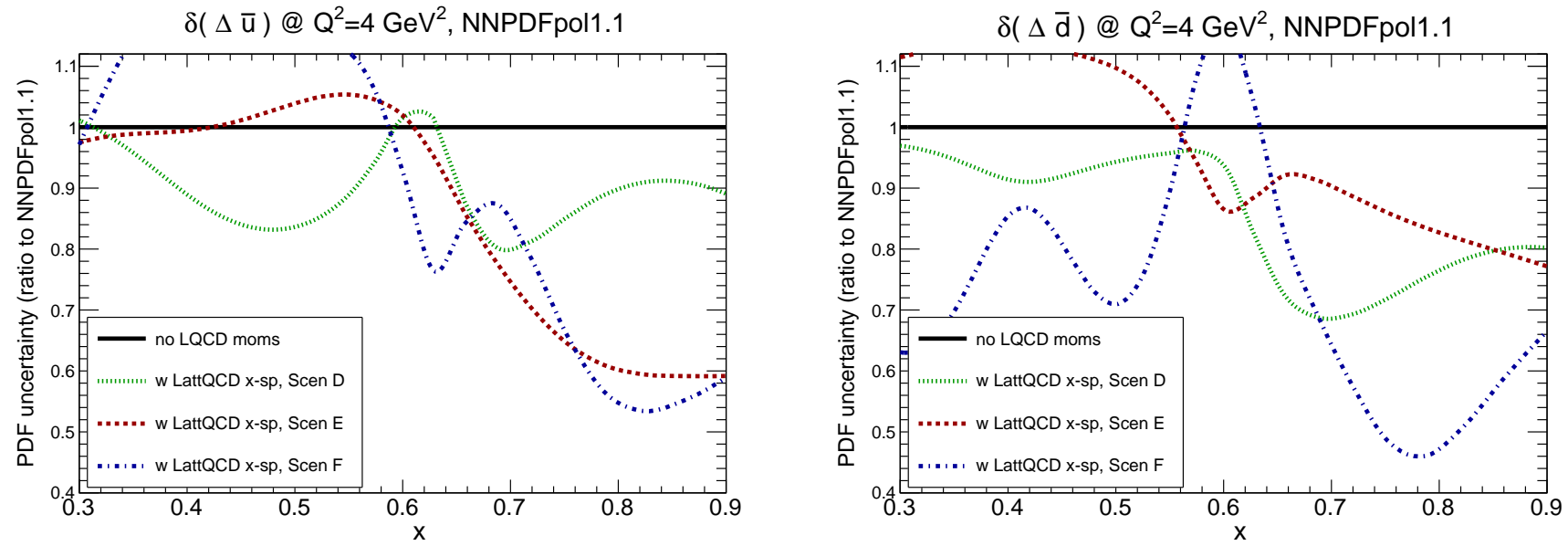

Figure 4.5: The ratio of PDF uncertainties to the original NNPDF3.1 (NNPDFpol1.1) in the fits where lattice-QCD pseudo-data on $x$-space PDFs have been added to the global unpolarized (polarized) analysis. Specifically, we show the impact on the PDF uncertainties in $\bar{u}$ and $\bar{d}$ at large- $x$ in the upper plots, with the corresponding comparison for $\Delta \bar{u}$ and $\Delta \bar{d}$ in the lower plots.

calculation, and on the values of the associated uncertainties $\delta_{L}^{(i)}$. The quantitative results depend on the choice of input PDF set and would vary if, for example, the input set were the HERAPDF2.0 set used for the Hessian profiling exercise of Sec. 4.1.3. Even with these caveats, our analysis makes clear that a direct computation of the isotriplet combination $x u-x d$ on the lattice has the potential to constrain the large- $x$ PDFs in a more significant way than corresponding PDF moment calculations, particularly in the unpolarized case. Given the importance of antiquark PDFs in the large- $x$ region for LHC phenomenology (especially for a high-luminosity run), pursuing these calculations should be high on the list of priorities for the lattice-QCD community.

\subsection{Discussion}

We conclude this section with a brief discussion of the main lessons that can be learned from this exercise, which provides the first quantitative estimate of the impact of present and future lattice-QCD calculations of PDF moments and $x$-space PDFs, for both polarized and unpolarized PDFs.

First, we have demonstrated that in the polarized case, even with current uncertainties, lattice-QCD calculations of selected PDF moments can impose sizable constraints on several important polarized quark combinations. This suggests that global polarized PDF analyses should consider including existing lattice-QCD calculations in their fits to constrain some of the least known quark combinations, such as the total strangeness. The situation is rather different in the unpolarized case, where a reduction of the 
current lattice-QCD uncertainties by a factor of between five and ten seems to be required to influence global fits. This difference arises because unpolarized PDFs are known with much higher precision than polarized PDFs, thanks to the much wider amount of experimental data sensitive to unpolarized PDFs, including the constraints from recent high-precision measurements at the LHC. Thus, in addition to the differences highlighted in Fig. 3.2, much more precise lattice-QCD calculations than in the polarized case need to be used to be competitive with current PDF fits.

Second, lattice-QCD calculations of the quark isotriplet combinations $x u-x d$ and $x \bar{u}-x \bar{d}$ would be instrumental in constraining quark PDFs at large $x$. Even a calculation with $\delta_{L} \simeq 10 \%$ uncertainties at large- $x$ would start to provide useful constraints on global fits. Moreover, we find that, in the unpolarized case, the information on the PDFs that could be derived from a direct $x$-space calculation from lattice-QCD is clearly superior to the information that can be obtained from PDF moments alone, at least for the subset of PDFs and moments used in the present exercise.

The profiling studies presented in this section could be extended in a number of directions. In the polarized case, one could include the current lattice-QCD values of the moments listed in Table 3.8 in global analyses: indeed, we have demonstrated that at the current level of uncertainties one expects to find some non-trivial constraints. In this respect, a crucial topic to investigate is the compatibility (or lack thereof) of the existing lattice-QCD numbers compared to constraints from experimental data. For both unpolarized and polarized PDFs, it would be interesting to include the effects of other moments and flavor combinations. Higher moments, in particular, typically probe regions of higher $x$, compared to lower moments, and in the large- $x$ regions uncertainties in the global-fit PDFs are more marked. One could also consider the effects of the quark combinations for which $x$-space calculations might be available, for example those related to the proton strangeness. Finally, a more refined analysis should include the theoretical correlations expected in lattice-QCD calculations, for instance, in the case of $x$-space calculations, one expects neighboring points in $x$ to be highly correlated.

\section{Outlook}

The study of the PDFs of the proton is an active interdisciplinary research field lying at the crossroads of high-energy, hadronic and nuclear physics, with important applications in astroparticle physics. In this white paper, we have reviewed our current knowledge of PDFs as determined from both the global analysis framework and from lattice-QCD computations. We have established a common language between the two communities, to facilitate interactions between them. We have presented a first systematic comparison between state-of-the-art lattice-QCD calculations of PDF moments and the corresponding results from global analyses both in the unpolarized and polarized cases. We have provided additional benchmark numbers from the global fits for the higher moments not used in this benchmark comparison, which can be used to validate future lattice-QCD calculations.

The main outcome of this white paper is the first quantitative study of the impact of lattice-QCD calculations in the global fits, based on both PDF moments and on Bjorken- $x$ dependence pseudo-data, assuming a number of different scenarios for the associated uncertainties. In the case of unpolarized PDFs, we have demonstrated that a reduction of the uncertainties of current lattice-QCD calculations is needed to provide any impact on global PDF fits. In the case of polarized PDFs, we have shown that current lattice-QCD calculations can provide useful input into global-PDF analyses. Although the studies presented here are still in an initial exploratory phase, they provide strong motivation for global fitters to begin consider incorporating lattice-QCD constraints into their global analyses.

The studies presented in this white paper can be extended in a number of directions.

First, we have restricted our benchmark comparison only to the lowest moments of polarized and unpolarized PDFs, whose various sources of systematic uncertainties have been computed with the greatest control. Future work should extend this comparison to higher PDF moments, which will 
have some impact on PDF fits, provided the precision and accuracy of lattice-QCD calculations keep improving.

Second, a similar benchmark exercise between global fit results and lattice-QCD calculations should be performed at the level of $x$-space calculations. It will be important to compare in detail the available lattice-QCD results with state-of-the-art global fits, to validate the former and thereby demonstrate to what extent lattice-QCD calculations of $x$-space PDFs can contribute to global fits.

Third, it should be possible to assess the impact of lattice-QCD calculations on other nonperturbative objects determined from global analyses of experimental data. Examples of these include the transversity (see Ref. [267] for a recent study), transverse-momentum dependent PDFs (TMDs), generalized PDFs (GPDs), or collinear PDFs for hadrons other than protons. All these quantities are known with much less precision than unpolarized and polarized PDFs, given that the corresponding experimental information is rather scarce. In this case, lattice-QCD calculations could have the potential to provide new information, without the need of high precision.

In summary, the aim of this study has been to build a bridge between the lattice-QCD and global-fit communities. Our final goal is for lattice-QCD calculations to provide novel inputs into polarized and unpolarized PDF fits. Precise lattice-QCD results could reduce the uncertainties of global PDF fits and/or discriminate between different sets. We hope this white paper motivates the lattice-QCD and global-fit communities to continue fruitful interactions to improve our knowledge of PDFs.

\section{Acknowledgments}

We are grateful to Jacqueline Gills and Michelle Bosher for their help in the organization of the workshop. We thank Rodolfo Sassot and Jacob Ethier for providing us with the DSSV14 predictions in Table C.3 and with the JAM17 predictions in Tables 3.6, C.2 and C.3. The workshop was partly supported by the European Research Council (ERC) via the Starting Grant PDF4BSM - Parton Distributions in the Higgs Boson Era. We also thank the Department of Energy's (DoE) Institute of Nuclear Theory (INT) at the University of Washington in Seattle for partial support during the completion of this work. This work was also partially funded by the U.S. DoE contract No. DE-AC05-06OR23177, under which Jefferson Science Associates, LLC operates Jefferson Lab. H.-W.L. is supported by the U.S. National Science Foundation (NSF) under Grant PHY 1653405; E.R.N. by the U.K. Science and Technology Facilities Council (STFC) via the Rutherford Grant ST/M003787/1 and the Consolidated Grant ST/P000630/1; K.O. by Jefferson Science Associates, LLC under U.S. DoE contract No. DEAC05-06OR23177, by U.S. DoE under Grant No. DE-FG02-04ER41302, and by STFC via the Consolidated Grant ST/P000681/1; F.I.O. and P.M.N. by the U.S. DoE under Grant No. DE-SC0010129; J.R. by the ERC via the Starting Grant PDF $4 B S M$ - Parton Distributions in the Higgs Boson Era and by the Dutch Organization for Scientific Research (NWO); A.A. acknowledges support by the U.S. DoE under contract No. DE-SC008791; A.B. and G.B. by the ERC via the Consolidator Grant 3DSPIN Mapping the proton in 3D; J.-W.C. partly by the Ministry of Science and Technology, Taiwan, under Grant No. 105-2112-M-002-017-MY3 and the Kenda Foundation; M.C. by the U.S. DoE, Office of Science, Office of Nuclear Physics, within the framework of the TMD Topical Collaboration, as well as by the NSF under Grant No. PHY-1714407; L.D.D. by the Royal Society, Wolfson Research Merit Award, grant No. WM140078: L.A.H.-L. and R.S.T. by the STFC via Grant awards ST/L000377/1 and ST/P000274/1; M.E. by the U.S. DoE, Office of Science, Office of Nuclear Physics through Grant DE-FG02-96ER40965 as well as through the TMD Topical Collaboration; C.J.M. by the U.S. DoE through Grant No. DE-FG02-00ER41132; T.I. by Science and Technology Commission of Shanghai Municipality (Grants No. 16DZ2260200) and in part by the DoE, Laboratory Directed Research and Development (LDRD) funding of BNL, under contract No. DE-EC0012704; C.-P.Y. by the NSF under Grant No. PHY-1417326. 


\section{A Definition of the PDF moments}

In this appendix, we summarize the conventions adopted in this paper to denote the moments of relevant unpolarized and polarized PDF combinations. We focus on the quantities which can be presently computed in lattice QCD, although those used for benchmarks in Sec. 3 are only a subset of them. In the equations below, we use the shorthand notation

$$
q^{ \pm} \equiv q \pm \bar{q} \quad \text { and } \quad \Delta q^{ \pm} \equiv \Delta q \pm \Delta \bar{q}, \quad q=u, d, s, c,
$$

for unpolarized and polarized PDFs respectively. We identify $\mu$ with the QCD factorization scale and $Q$ with the characteristic scale of a given hard-scattering process. The use of the following notation is strongly recommended for any comparison between lattice-QCD computations and global-fit determinations of PDF moments.

- Unpolarized moments.

1. The first moment of the total $u^{+}-d^{+}$PDF combination

$$
\left.\langle x\rangle_{u^{+}-d^{+}}\left(\mu^{2}\right)\right|_{\mu^{2}=Q^{2}}=\int_{0}^{1} d x x\left\{u\left(x, Q^{2}\right)+\bar{u}\left(x, Q^{2}\right)-d\left(x, Q^{2}\right)-\bar{d}\left(x, Q^{2}\right)\right\} .
$$

2. The second moment of the valence $u^{-}-d^{-}$PDF combination

$$
\left.\left\langle x^{2}\right\rangle_{u^{-}-d^{-}}\left(\mu^{2}\right)\right|_{\mu^{2}=Q^{2}}=\int_{0}^{1} d x x^{2}\left\{u\left(x, Q^{2}\right)-\bar{u}\left(x, Q^{2}\right)-d\left(x, Q^{2}\right)+\bar{d}\left(x, Q^{2}\right)\right\} .
$$

3. The first moment of the individual quark $q^{+}$total PDF combination

$$
\left.\langle x\rangle_{q^{+}=u^{+}, d^{+}, s^{+}, c^{+}}\left(\mu^{2}\right)\right|_{\mu^{2}=Q^{2}}=\int_{0}^{1} d x x\left\{q\left(x, Q^{2}\right)+\bar{q}\left(x, Q^{2}\right)\right\} .
$$

4. The second moment of the individual quark $q^{-}$valence PDF combination

$$
\left.\left\langle x^{2}\right\rangle_{q^{-}=u^{-}, d^{-}, s^{-}, c^{-}}\left(\mu^{2}\right)\right|_{\mu^{2}=Q^{2}}=\int_{0}^{1} d x x^{2}\left\{q\left(x, Q^{2}\right)-\bar{q}\left(x, Q^{2}\right)\right\} .
$$

5. The first moment of the gluon PDF

$$
\left.\langle x\rangle_{g}\left(\mu^{2}\right)\right|_{\mu^{2}=Q^{2}}=\int_{0}^{1} d x x g\left(x, Q^{2}\right) .
$$

- Polarized moments.

1. The zeroth moment of the total $u^{+}-d^{+}$PDF combination

$$
\left.\langle 1\rangle_{\Delta u^{+}-\Delta d^{+}}\left(\mu^{2}\right)\right|_{\mu^{2}=Q^{2}}=\int_{0}^{1} d x\left\{\Delta u\left(x, Q^{2}\right)+\Delta \bar{u}\left(x, Q^{2}\right)-\Delta d\left(x, Q^{2}\right)-\Delta \bar{d}\left(x, Q^{2}\right)\right\} .
$$

2. The first moment of the valence $u^{-}-d^{-}$PDF combination

$$
\left.\langle x\rangle_{\Delta u^{-}-\Delta d^{-}}\left(\mu^{2}\right)\right|_{\mu^{2}=Q^{2}}=\int_{0}^{1} d x x\left\{\Delta u\left(x, Q^{2}\right)-\Delta \bar{u}\left(x, Q^{2}\right)-\Delta d\left(x, Q^{2}\right)+\Delta \bar{d}\left(x, Q^{2}\right)\right\}
$$


3. The zeroth moment of the individual quark $q^{+}$total PDF combination

$$
\left.\langle 1\rangle_{q^{+}=\Delta u^{+}, \Delta d^{+}, \Delta s^{+}, \Delta c^{+}}\left(\mu^{2}\right)\right|_{\mu^{2}=Q^{2}}=\int_{0}^{1} d x\left\{\Delta q\left(x, Q^{2}\right)+\Delta \bar{q}\left(x, Q^{2}\right)\right\} .
$$

4. The first moment of the individual quark $q^{-}$valence PDF combination

$$
\left.\langle x\rangle_{\Delta q^{-}=\Delta u^{-}, \Delta d^{-}, \Delta s^{-}, \Delta c^{-}}\left(\mu^{2}\right)\right|_{\mu^{2}=Q^{2}}=\int_{0}^{1} d x x\left\{\Delta q\left(x, Q^{2}\right)-\Delta \bar{q}\left(x, Q^{2}\right)\right\} .
$$

Some of these moments have a direct physical interpretation, see Sec. 2.1. For instance, Eq. (A.4) and Eq. (A.9) correspond respectively to the proton's momentum and spin fractions carried by a given quark flavor (and its corresponding antiquark) at the scale $\mu^{2}=Q^{2}$. Higher moments and/or moments of other flavor combinations are readily computable from any phenomenological PDF set. We do not consider them though, as the corresponding lattice-QCD computations are outside the current reach.

\section{B PDF moments from lattice QCD}

In this appendix, we summarize additional results for the moments of unpolarized and polarized PDFs from lattice QCD that were not discussed in Sec. 3.1.2, either because the calculations were performed in the quenched approximation, or because they were not extrapolated to the physical pion mass.

- In Table B.1, we show the first moments of unpolarized PDFs $\langle x\rangle_{u^{+-d^{+}}},\langle x\rangle_{q^{+}}$and $\langle x\rangle_{g}$ that were not included in Table 3.1.

- In Table B.2, we show the second moments of unpolarized PDFs $\left\langle x^{2}\right\rangle_{u^{-}-d^{-}},\left\langle x^{2}\right\rangle_{u^{-}}$and $\left\langle x^{2}\right\rangle_{d^{-}}$.

- In Table B.3, we show the zeroth moments of polarized PDFs $\langle 1\rangle_{\Delta u^{+}},\langle 1\rangle_{\Delta d^{+}}$and $\langle 1\rangle_{\Delta s^{+}}$that were not included in Table 3.3.

- In Table B.4, we show the first moments of polarized PDFs $\langle x\rangle_{\Delta u^{-}-\Delta d^{-}},\langle x\rangle_{\Delta u^{-}}$and $\langle x\rangle_{\Delta d^{-}}$that were not included in Table 3.4 .

All results are displayed at $\mu^{2}=4 \mathrm{GeV}^{2}$. The characterization of each source of systematic uncertainty follows the conventions delineated in Sec. 3.1.2, to which the reader is referred for the meaning of each symbol in Tables B.1-B.4. Moments are denoted according to the notation introduced in Appendix A.

We also provide tables with full bibliographic details.

- In Table B.5, for the axial coupling $g_{A} \equiv\langle 1\rangle_{\Delta u^{+}-\Delta d^{+}}$. We do not include quenched results, perturbatively renormalized results, and conference proceedings results.

- In Table B.6, for the non-isovector quark spins.

- In Table B.7, for $\langle x\rangle_{u^{+-} d^{+}}$. We omit quenched and non-renormalized results.

- In Table B.8, for the non-isovector momentum fractions.

- In Table B.9, for $\langle x\rangle_{\Delta u^{-}-\Delta d^{-}}$.

- In Table B.10 for higher moments of unpolarized and polarized PDFs.

A representative subset of the results contained in Tables 3.1, 3.3, 3.4, B.1, B.3 and B.4 is displayed in Fig. B.1. Specifically, we show from left to right and from top to bottom results for $\langle x\rangle_{u^{+}-d^{+}},\langle x\rangle_{q^{+}}$, $\langle x\rangle_{g},\langle 1\rangle_{\Delta s^{+}},\langle 1\rangle_{\Delta q^{+}}$and $\langle x\rangle_{\Delta u^{-}-\Delta d^{-}}$; see the corresponding entries of each table for details. 


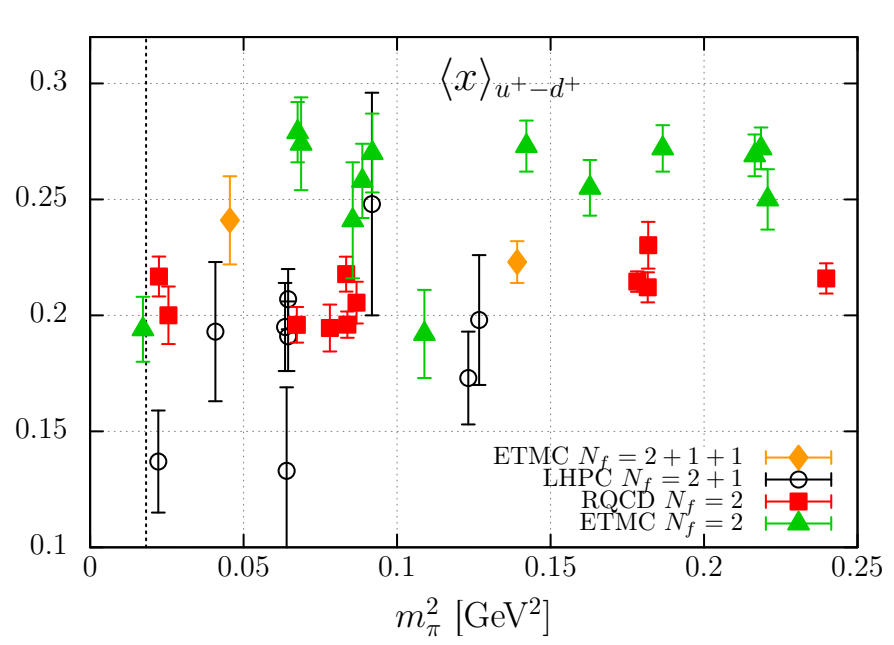

(a)

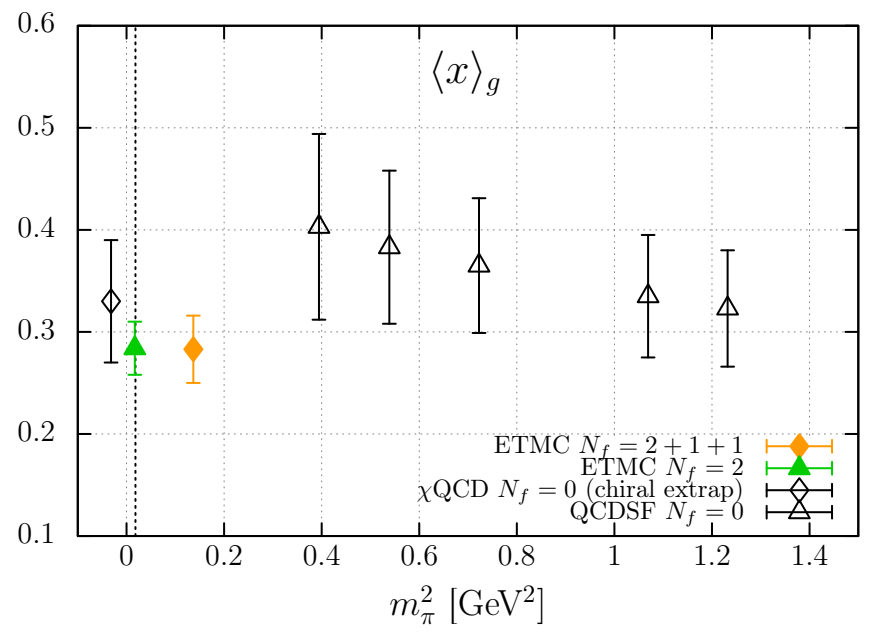

(c)

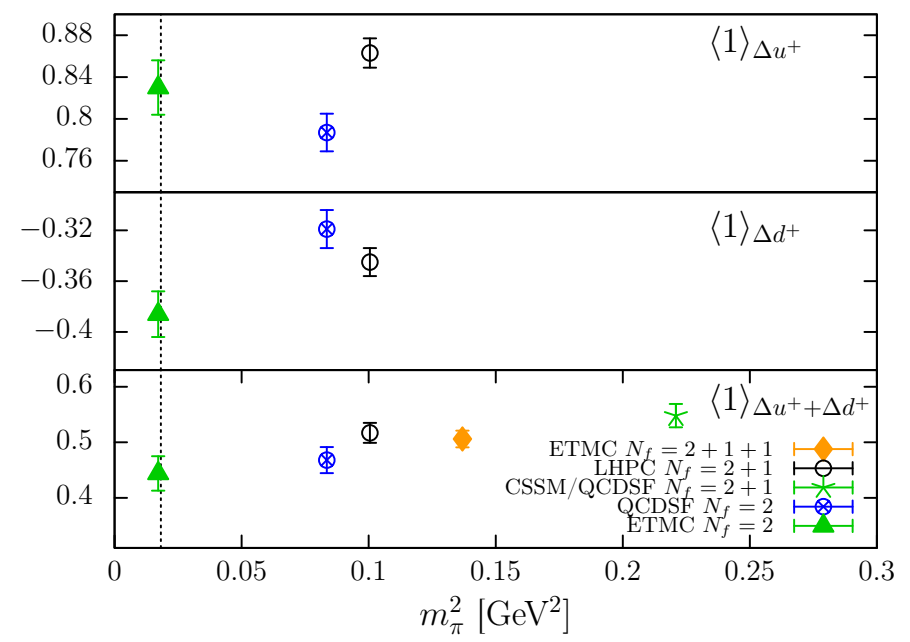

(e)

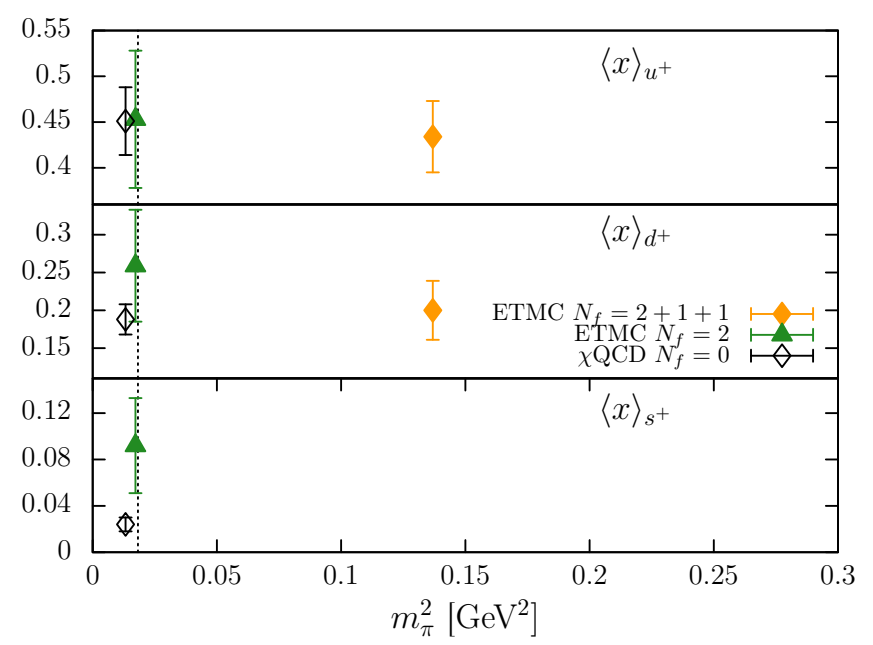

(b)

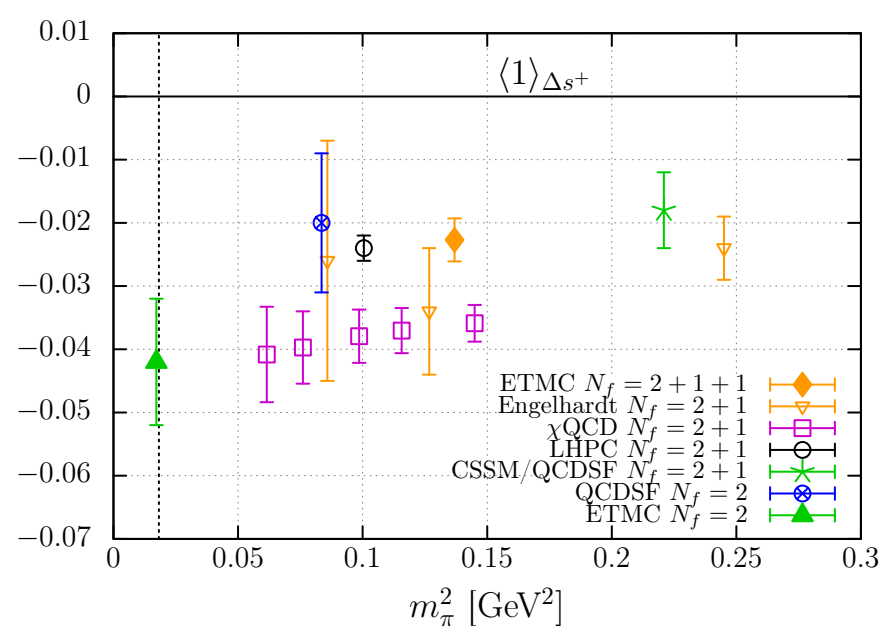

(d)

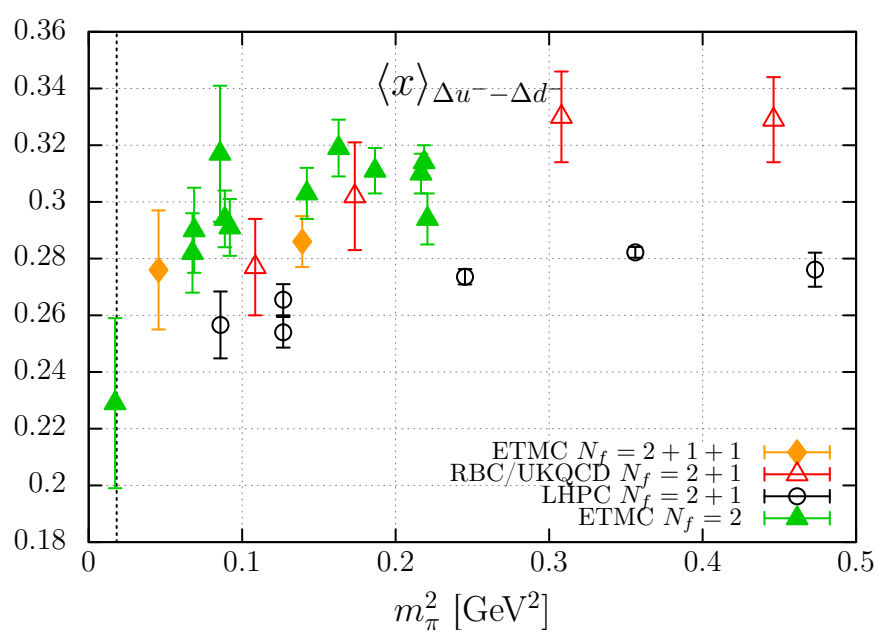

(f)

Figure B.1: Comparison of lattice-QCD results for the zeroth and first moments of unpolarized and polarized PDFs. From left to right and from top to bottom, we show results for $\langle x\rangle_{u^{+}-d^{+}},\langle x\rangle_{q^{+}},\langle x\rangle_{g},\langle 1\rangle_{\Delta s^{+}},\langle 1\rangle_{\Delta q^{+}}$ and $\langle x\rangle_{\Delta u^{-}-\Delta d^{-}}$; see Tables 3.1, 3.3, 3.4, B.1, B.3 and B.4 for details. 


\begin{tabular}{|c|c|c|c|c|c|c|c|c|c|c|c|}
\hline Mom. & Collab. & Ref. & $N_{f}$ & Status & Disc $[\mathrm{fm}]$ & $\mathrm{QM}$ & $\mathrm{FV}$ & Ren & $\mathrm{ES}$ & & \\
\hline \multirow[t]{3}{*}{$\langle x\rangle_{u^{+}-d^{+}}$} & ETMC 15 & {$[261]$} & $2+1+1$ & $\mathrm{P}$ & $0.06,0.08$ & - & $\boldsymbol{\square}, \star$ & $\star, \star$ & $\square, \star$ & & Fig. B.1 (a) \\
\hline & ETMC 15 & {$[261]$} & 2 & $\mathrm{P}$ & $0.06-0.09$ & - & O & $\star$ & $\boldsymbol{\square}$ & & Fig. B.1 (a) \\
\hline & RQCD 14 & {$[249]$} & 2 & $\mathrm{P}$ & $0.06-0.08$ & - & O & $\star$ & O & & Fig. B.1 (a) \\
\hline \multirow[t]{4}{*}{$\langle x\rangle_{q^{+}}$} & ETMC 13 & {$[268]$} & $2+1+1$ & $\mathrm{P}$ & 0.08 & - & $\star$ & $\star$ & $\star$ & $\&$ & Fig. B.1 (b) \\
\hline & $\chi \mathrm{QCD} 13$ & [269] & 0 & $\mathrm{P}$ & $\boldsymbol{\square}$ & $\mathbf{\square}$ & $\mathbf{\square}$ & O & $\mathbf{\square}$ & $\dagger t$ & $\langle x\rangle_{u^{+}}=0.451(37)$ \\
\hline & $\chi \mathrm{QCD} 13$ & {$[269]$} & 0 & $\mathrm{P}$ & $\boldsymbol{\square}$ & $\boldsymbol{\square}$ & $\mathbf{\square}$ & O & $\boldsymbol{\square}$ & $\dagger t$ & $\langle x\rangle_{d^{+}}=0.188(20)$, \\
\hline & $\chi \mathrm{QCD} 13$ & {$[269]$} & 0 & $\mathrm{P}$ & $\boldsymbol{\square}$ & $\mathbf{\square}$ & $\boldsymbol{\square}$ & O & $\boldsymbol{\square}$ & $\dagger t$ & $\langle x\rangle_{s^{+}}=0.024(6)$ \\
\hline \multirow[t]{3}{*}{$\langle x\rangle_{g}$} & ETMC 13 & {$[270]$} & $2+1+1$ & $\mathrm{P}$ & 0.08 & - & $\star$ & 0 & $\star$ & & Fig. B.1 (c) \\
\hline & $\chi \mathrm{QCD} 13$ & [269] & 0 & $\mathrm{P}$ & $\boldsymbol{\square}$ & $\square$ & $\square$ & O & $\star$ & $\ddagger$ & $0.334(55)$ \\
\hline & QCDSF 12 & [111] & 0 & $\mathrm{P}$ & $\boldsymbol{\square}$ & $\boldsymbol{\square}$ & $\star$ & $\star$ & - & $\dagger$ & $0.43(7)(5)$ \\
\hline
\end{tabular}

\footnotetext{
\& Nonsinglet renormalization is applied.

$\dagger$ The lightest $m_{\pi}$ has $L m_{\pi} \geq 4.0$, however, $L \sim 1.6 \mathrm{fm}$.

$\ddagger$ The connected contribution is only evaluated at one $t_{\text {sep }}$.
}

Table B.1: Status of current lattice-QCD calculations of the first moments of unpolarized PDFs. All results are quoted at $\mu^{2}=4 \mathrm{GeV}^{2}$. We use the abbreviations Disc (discretization), QM (quark mass), FV (finite volume), Ren (renormalization) and ES (excited states) to denote the corresponding sources of uncertainty.

\begin{tabular}{|c|c|c|c|c|c|c|c|c|c|c|c|}
\hline Mom. & Collab. & Ref. & $N_{f}$ & Status & Disc & $\mathrm{QM}$ & FV & Ren & ES & & \\
\hline \multirow[t]{3}{*}{$\left\langle x^{2}\right\rangle_{u^{-}-d^{-}}$} & LHPC and SESAM 02 & {$[271]$} & 2 & $\mathrm{P}$ & $\boldsymbol{\square}$ & $\square$ & $\mathbf{\square}$ & O & $\mathbf{\square}$ & & $0.145(69)$ \\
\hline & QCDSF 05 & {$[91]$} & 0 & $\mathrm{P}$ & $\mathbf{\square}$ & $\boldsymbol{\square}$ & $\boldsymbol{\square}$ & $\star$ & $\mathbf{\square}$ & & $0.083(17)$ \\
\hline & LHPC and SESAM 02 & {$[271]$} & 0 & $\mathrm{P}$ & $\mathbf{\square}$ & $\boldsymbol{\square}$ & $\square$ & O & $\mathbf{\square}$ & & $0.090(68)$ \\
\hline$\left\langle x^{2}\right\rangle_{u^{-}}$ & $\chi \mathrm{QCD} 09$ & {$[272]$} & 0 & $\mathrm{P}$ & $\mathbf{\square}$ & घ & $\boldsymbol{\square}$ & O & $\boldsymbol{\square}$ & $*$ & $0.117(18)$ \\
\hline$\left\langle x^{2}\right\rangle_{d^{-}}$ & $\chi \mathrm{QCD} 09$ & {$[272]$} & 0 & $\mathrm{P}$ & $\boldsymbol{\square}$ & $\boldsymbol{\square}$ & $\mathbf{\square}$ & O & $\mathbf{\square}$ & $*$ & $0.052(9)$ \\
\hline
\end{tabular}

* Only the connected contribution is included.

Table B.2: Same as Table B.1, but for second moments of unpolarized PDFs.

\begin{tabular}{|c|c|c|c|c|c|c|c|c|c|c|c|}
\hline Mom. & Collab. & Ref. & $N_{f}$ & Status & Disc $[\mathrm{fm}]$ & $\mathrm{QM}$ & FV & Ren & ES & & \\
\hline \multirow[t]{4}{*}{$\langle 1\rangle_{\Delta u^{+}, \Delta d^{+}}$} & ETMC 13 & {$[268]$} & $2+1+1$ & $\mathrm{P}$ & 0.08 & - & $\star$ & $\star$ & $\star$ & $\&$ & Fig. B.1 (e) \\
\hline & LHPC 17 & {$[273]$} & $2+1$ & $\mathrm{P}$ & 0.11 & - & $\star$ & $\star$ & $\star$ & & Fig. B.1 (e) \\
\hline & QCDSF/CSSM 15 & {$[113]$} & $2+1$ & $\mathrm{P}$ & 0.07 & - & $\star$ & $\star$ & $\star$ & & Fig. B.1 (e) \\
\hline & QCDSF 11 & {$[274]$} & 2 & $\mathrm{P}$ & 0.07 & - & $\star$ & $\star$ & $\mathbf{\square}$ & $\$$ & Fig. B.1 (e) \\
\hline \multirow[t]{4}{*}{$\langle 1\rangle_{\Delta s^{+}}$} & ETMC 13 & {$[268]$} & $2+1+1$ & $\mathrm{P}$ & 0.08 & - & $\star$ & $\star$ & $\star$ & $\&$ & Fig. B.1 (d) \\
\hline & LHPC 17 & {$[273]$} & $2+1$ & $\mathrm{P}$ & 0.11 & - & $\star$ & $\star$ & $\star$ & & Fig. B.1 (d) \\
\hline & QCDSF/CSSM 15 & {$[113]$} & $2+1$ & $\mathrm{P}$ & 0.07 & - & $\star$ & $\star$ & $\star$ & & Fig. B.1 (d) \\
\hline & QCDSF 11 & {$[274]$} & 2 & $\mathrm{P}$ & 0.07 & - & $\star$ & $\star$ & $\star$ & $\$$ & Fig. B.1 (d) \\
\hline
\end{tabular}

\& Nonsinglet renormalization is applied.

$\$$ The mixing with $\langle x\rangle_{q^{+}}$is small so only the excited state analysis for $\langle x\rangle_{g}$ is considered.

Table B.3: Same as Table B.1, but for zeroth moments of polarized PDFs. 


\begin{tabular}{|c|c|c|c|c|c|c|c|c|c|c|c|}
\hline Mom. & Collab. & Ref. & $N_{f}$ & Status & Disc [fm] & $\mathrm{QM}$ & $\mathrm{FV}$ & Ren & ES & & \\
\hline \multirow[t]{2}{*}{$\langle x\rangle_{\Delta u^{-}-\Delta d^{-}}$} & ETMC 15 & {$[261]$} & $2+1+1$ & $\mathrm{P}$ & $0.06,0.08$ & - & $\square, \star$ & $\star, \star$ & $\square, \star$ & & Fig. B.1 (f) \\
\hline & ETMC 15 & {$[261]$} & 2 & $\mathrm{P}$ & $0.06-0.09$ & - & 0 & $\star$ & $\boldsymbol{\square}$ & & Fig. B.1 (f) \\
\hline$\langle x\rangle_{\Delta u^{-}}$ & ETMC 13 & {$[268]$} & $2+1+1$ & $\mathrm{P}$ & 0.08 & $373 \mathrm{MeV}$ & $\star$ & $\star$ & $\star$ & $\&$ & $0.214(11)$ \\
\hline$\langle x\rangle_{\Delta d^{-}}$ & ETMC 13 & {$[268]$} & $2+1+1$ & $\mathrm{P}$ & 0.08 & $373 \mathrm{MeV}$ & $\star$ & $\star$ & $\star$ & $\&$ & $0.083(11)$ \\
\hline
\end{tabular}

$\&$ Nonsinglet renormalization is applied.

Table B.4: Same as Table B.1, but for first moments of polarized PDFs.

\begin{tabular}{|c|c|c|c|c|c|}
\hline Ref. & Sea quarks & Valence quarks & Renormalization & $N_{\Delta t}$ & $m_{\pi}(\mathrm{MeV})$ \\
\hline Mainz '17b* [252] & 2 clover & clover & Schrödinger functional & $4-6$ & $193-473$ \\
\hline ETMC '17b [253] & 2 clover-TM & clover-TM & Rome-Southampton & 3 & 131 \\
\hline CalLat '17b [250] & $2+1+1$ staggered & domain wall & Rome-Southampton & all & $131-313$ \\
\hline LHPC '17 [273] & $2+1$ clover & clover & Rome-Southampton & 5 & 317 \\
\hline NME '17 [275] & $2+1$ clover & clover & Rome-Southampton & $1^{* *}, 4-5$ & $172-285$ \\
\hline Mainz '17a [276] & 2 clover & clover & Schrödinger functional & $4-6$ & $193-456$ \\
\hline Dragos et al. '16 [277] & 3 clover & clover & Rome-Southampton & $1,2^{* *}, 5$ & 460 \\
\hline PNDME '16 [251] & $2+1+1$ staggered & clover & Rome-Southampton & $3-5$ & $128-319$ \\
\hline$\chi \mathrm{QCD}^{\prime} 16[278]$ & $2+1$ domain wall & overlap & $Z_{A} / Z_{V}=1$ & 3 & 330 \\
\hline \multirow[t]{3}{*}{ ETMC '15b [261] } & 2 clover-TM & superseded by $\mathrm{E}$ & TMC '17 & & \\
\hline & 2 twisted mass & twisted mass & Rome-Southampton & 1 & $262-470$ \\
\hline & $2+1+1$ twisted mass & twisted mass & & 1,4 & 213,373 \\
\hline RQCD '15 [254] & 2 clover & clover & Rome-Southampton & $1-5$ & $150-490$ \\
\hline PNDME '14 [279] & \multicolumn{5}{|c|}{ superseded by PNDME '16 } \\
\hline QCDSF '14 [255] & 2 clover & clover & $g_{A} / f_{\pi} \times f_{\pi}^{\text {phys }}$ & 1,5 & $157-1591$ \\
\hline LHPC '14 [247] & $2+1$ clover & clover & Rome-Southampton & 3 & $149-356$ \\
\hline ETMC '13 [280] & superseded by ETMC & '15b & & & \\
\hline CSSM '13 [281] & $2+1$ clover & clover & Schrödinger functional & $1^{* * \dagger}$ & 290 \\
\hline Mainz '12 [282] & \multicolumn{5}{|c|}{ superseded by Mainz '17b } \\
\hline ETMC '11 [283] & \multicolumn{5}{|c|}{ superseded by ETMC '15b } \\
\hline LHPC '10 [260] & $2+1$ staggered & domain wall & $A_{\mu} / \mathcal{A}_{\mu}$ ratio & $1-2$ & $293-758$ \\
\hline RBC+UKQCD '09 [284] & $2+1$ domain wall & domain wall & $Z_{A} / Z_{V}=1$ & 1 & $329-668$ \\
\hline RBC+UKQCD '08 [285] & \multicolumn{5}{|c|}{ superseded by RBC+UKQCD ’09 } \\
\hline RBC ’08 [286] & 2 domain wall & domain wall & $Z_{A} / Z_{V}=1$ & $1-2$ & $493-695$ \\
\hline LHPC '08 [287] & \multicolumn{5}{|c|}{ superseded by LHPC '10 } \\
\hline Alexandrou et al. '07 [288] & 2 Wilson & Wilson & Rome-Southampton & 1 & $384-691$ \\
\hline LHPC '06 [289] & \multicolumn{5}{|c|}{ superseded by LHPC '10 } \\
\hline QCDSF '06 [290] & \multicolumn{5}{|c|}{ superseded by QCDSF '14 } \\
\hline
\end{tabular}

* Preprint.

** A variationally optimized interpolating operator is employed.

${ }^{\dagger}$ Carried out with a single fixed source-operator separation and all source-sink separations.

Table B.5: Full details of lattice-QCD calculations of the axial coupling $g_{A} \equiv\langle 1\rangle_{\Delta u^{+}-\Delta d^{+}}$. We omit quenched results, perturbatively renormalized results, and conference proceedings. 


\begin{tabular}{|c|c|c|c|c|}
\hline Ref. & Flavors & Sea quarks & Valence quarks & Renormalization \\
\hline ETMC '17b [253] & $u, d, s, c$ & 2 clover-TM & clover-TM & Rome-Southampton \\
\hline ETMC '17c [248] & $u, d, s$ & 2 clover-TM & clover-TM & Rome-Southampton \\
\hline$\chi \mathrm{QCD}^{\prime} 17 \mathrm{~b}[257]$ & $s, c$ & $2+1$ domain wall & overlap & single-flavor anomalous WI \\
\hline LHPC '17 [273] & $u, d, s$ & $2+1$ clover & clover & Rome-Southampton \\
\hline CSSM and & $u+d+s$ & $2+1,3$ clover & clover & Rome-Southampton \\
\hline QCDSF/UKQCD '15 [113] & conn. / disc. & & & \\
\hline ETMC '14 [268] & $u+d, s$ & $2+1+1$ twisted mass & twisted mass & nonsinglet Rome-Southampton \\
\hline Engelhardt '12 [258] & $s$ & $2+1$ staggered & domain wall & nonsinglet $A_{\mu} / \mathcal{A}_{\mu}$ ratio \\
\hline QCDSF '12 [274] & $u, d, s$ & 2 clover & clover & $\begin{array}{l}\text { nonsinglet Rome-Southampton } \\
+ \text { two-loop singlet-nonsinglet }\end{array}$ \\
\hline Babich et al. '10 [291] & $s$ & 2 aniso-clover & aniso-clover & none \\
\hline SESAM '99 [292] & $u, d, s$ & 2 Wilson & Wilson & one loop \\
\hline$\chi \mathrm{QCD}^{\prime} 95[293]$ & $u, d, s$ & quenched & Wilson & one loop \\
\hline Fukugita et al. '95 [294] & $u, d, s$ & quenched & Wilson & one loop \\
\hline Gupta and Mandula '94 [295] & singlet** & quenched & Wilson & anomalous Ward identity \\
\hline Allés et al. '94 [296] & singlet** & quenched & Wilson & anomalous Ward identity \\
\hline Altmeyer et al. '94 [297] & singlet & 4 staggered & staggered & anomalous Ward identity \\
\hline Mandula and Ogilvie '93 [298] & $s^{* *}$ & quenched & Wilson & none \\
\hline
\end{tabular}

* Preprint.

** No signal available.

Table B.6: Full details of lattice-QCD calculations of the non-isovector quark spins. The earliest results are summarized in Ref. [299].

\begin{tabular}{|c|c|c|c|c|c|}
\hline Ref. & Sea quarks & Valence quarks & Renormalization & $N_{\Delta t}$ & $m_{\pi}(\mathrm{MeV})$ \\
\hline ఒQCD '16 [278] & $2+1$ domain wall & overlap & one loop & 3 & 330 \\
\hline \multirow[t]{3}{*}{ ETMC '15b [261] } & 2 clover-TM & clover-TM & \multirow[t]{3}{*}{ Rome-Southampton } & 3 & 131 \\
\hline & 2 twisted mass & twisted mass & & 1 & $262-470$ \\
\hline & $2+1+1$ twisted mass & twisted mass & & 1,5 & 213,373 \\
\hline ETMC '15a [300] & $2+1+1$ twisted mass & twisted mass & Rome-Southampton & 1 & $302-466$ \\
\hline RQCD '14 [249] & 2 clover & clover & Rome-Southampton & $1-6$ & $149-490$ \\
\hline LHPC '14 [247] & $2+1$ clover & clover & Rome-Southampton & 3 & $149-356$ \\
\hline ETMC '13 [280] & \multicolumn{5}{|c|}{ superseded by ETMC '15b } \\
\hline RQCD '12 [301] & \multicolumn{5}{|c|}{ superseded by RQCD '14 } \\
\hline ETMC '11 [283] & \multicolumn{5}{|c|}{ superseded by ETMC '15b } \\
\hline QCDSF/UKQCD '11* [302] & 2 clover & clover & Rome-Southampton & 1 & $170-670$ \\
\hline LHPC ' $11^{*}[303]$ & $2+1$ domain wall & domain wall & Rome-Southampton & 1 & $297-403$ \\
\hline LHPC '10 [260] & $2+1$ staggered & domain wall & one-loop $Z_{\mathcal{O}} / Z_{A}$ & $1-2$ & $293-758$ \\
\hline RBC-UKQCD '10 [259] & $2+1$ domain wall & domain wall & Rome-Southampton & 1 & $329-668$ \\
\hline RBC '08 [286] & 2 domain wall & domain wall & Rome-Southampton & $1-2$ & $493-695$ \\
\hline LHPC '08 [287] & \multicolumn{5}{|c|}{ superseded by LHPC '10 } \\
\hline LHPC and & 2 Wilson & Wilson & one loop & $1-2$ & 490 \\
\hline SESAM '02 [271] & \multicolumn{5}{|l|}{ and quenched } \\
\hline
\end{tabular}

* Conference proceedings.

Table B.7: Full details of lattice-QCD calculations of $\langle x\rangle_{u^{+-} d^{+}}$. We omit quenched and non-renormalized results. 


\begin{tabular}{|c|c|c|c|c|}
\hline Ref. & Flavors & Sea quarks & Valence quarks & Renormalization \\
\hline ETMC '17a [270] & $g$ & $\begin{array}{l}2+1+1 \text { twisted mass } \\
2 \text { clover-TM }\end{array}$ & $\begin{array}{l}\text { twisted mass } \\
\text { clover-TM }\end{array}$ & one loop \\
\hline ETMC '17c [248] & $u, d, s, g$ & 2 clover-TM & clover-TM & $\begin{array}{l}\text { Rome-Southampton }(q) \\
\text { one-loop }(g)\end{array}$ \\
\hline ETMC '15a [300] & $u+d-2 s$ & $2+1+1$ twisted mass & twisted mass & Rome-Southampton \\
\hline ETMC '14 [268] & $u+d$ & $2+1+1$ twisted mass & twisted mass & nonsinglet \\
\hline $\begin{array}{l}\chi Q C D \text { '15 [269] } \\
\text { QCDSF-UKQCD '12 [111] }\end{array}$ & $\begin{array}{l}u, d, s, g \\
g\end{array}$ & $\begin{array}{l}\text { quenched } \\
\text { quenched }\end{array}$ & $\begin{array}{l}\text { Wilson } \\
\text { clover }\end{array}$ & $\begin{array}{l}\text { Rome-Southampton } \\
\text { sum rule + one-loop } \\
\text { nonperturbative }\end{array}$ \\
\hline
\end{tabular}

* Preprint.

Table B.8: Full details of lattice-QCD calculations of the non-isovector momentum fractions.

\begin{tabular}{lllll}
\hline Ref. & Sea quarks & Valence quarks & Renormalization & $N_{\Delta t}$ \\
\hline ETMC '15b [261] & 2 clover-TM & clover-TM & Rome-Southampton & 3 \\
& 2 twisted mass & twisted mass & & 1 \\
& $2+1+1$ twisted mass & twisted mass & & 1 or 4 \\
ETMC '13 [280] & superseded by ETMC '15b & & \\
ETMC '11 [283] & superseded by ETMC '15b & Rome-Southampton & 1 \\
QCDSF /UKQCD '11* [302] & 2 clover & clover & one-loop $Z_{\mathcal{O}} / Z_{A}$ & $1-2$ \\
LHPC '10 [260] & $2+1$ staggered & domain wall & Rome-Southampton & 1 \\
RBC-UKQCD '10 [259] & $2+1$ domain wall & domain wall & Rome-Southampton & $1-2$ \\
RBC '08 [286] & 2 domain wall & domain wall & Roum & \\
LHPC '08 [287] & superseded by LHPC '10 & & $1-2$ \\
LHPC and & 2 Wilson & Wilson & & \\
SESAM '02 [271] & and quenched & & one loop & 1 \\
QCDSF '97 [304] & quenched & Wilson & & \\
\hline
\end{tabular}

* Conference proceedings.

Table B.9: Full details of lattice-QCD calculations of $\langle x\rangle_{\Delta u^{-}-\Delta d^{-}}$. 


\begin{tabular}{|c|c|c|c|c|}
\hline Ref. & Observables & Sea quarks & Valence quarks & Renormalization \\
\hline LHPC ' $10^{\dagger}[260]$ & $\begin{array}{l}\langle x\rangle_{u^{+}-d^{+}},\left\langle x^{2}\right\rangle_{u^{-}-d^{-}} \\
g_{A},\langle x\rangle_{\Delta u^{-}-\Delta d^{-}},\left\langle x^{2}\right\rangle_{\Delta u^{+}-\Delta d^{+}}\end{array}$ & $2+1$ staggered & domain wall & one-loop $Z_{\mathcal{O}} / Z_{A}$ \\
\hline$\chi \mathrm{QCD}$ '09 [272] & $\begin{array}{l}\left.\langle x\rangle_{u^{+}, d^{+}, s^{+}} \text {(superseded by } \chi \mathrm{QCD}{ }^{\prime} 15\right), \\
\left\langle x^{2}\right\rangle_{u^{-}, d^{-}, s^{-}} \\
\text {superseded by LHPC '10 }\end{array}$ & quenched & Wilson & one loop \\
\hline QCDSF '05c [305] & $\left\langle x^{2}\right\rangle_{\Delta u^{+}-\Delta d^{+}}$ & 2 clover & clover & Rome-Southampton \\
\hline $\mathrm{QCDSF}^{\prime} 05 \mathrm{~b}[91]$ & $\langle x\rangle_{u^{+}-d^{+}},\left\langle x^{2}\right\rangle_{u^{-}-d^{-}},\left\langle x^{3}\right\rangle_{u^{+}-d^{+}}$ & quenched & clover & Rome-Southampton \\
\hline QCDSF '05a* [306] & $\langle x\rangle_{u^{+}-d^{+}},\left\langle x^{2}\right\rangle_{u^{-}-d^{-}},\left\langle x^{3}\right\rangle_{u^{+-d^{+}}}$ & 2 clover & clover & one loop \\
\hline $\begin{array}{l}\text { LHPC and } \\
\text { SESAM '02 [271] }\end{array}$ & $\begin{array}{l}\langle x\rangle_{u^{+}-d^{+}},\left\langle x^{2}\right\rangle_{u^{-}-d^{-}},\left\langle x^{3}\right\rangle_{u^{+-}-d^{+}} \\
g_{A},\langle x\rangle_{\Delta u^{-}-\Delta d^{-}},\left\langle x^{2}\right\rangle_{\Delta u^{+}-\Delta d^{+}}\end{array}$ & $\begin{array}{l}2 \text { Wilson } \\
\text { and quenched }\end{array}$ & Wilson & one loop \\
\hline $\mathrm{QCDSF}^{\prime} 01[307]$ & $\left\langle x^{2}\right\rangle_{\Delta u^{+}-\Delta d^{+}}$ & quenched & clover & Rome-Southampton \\
\hline QCDSF '96 [98] & $\begin{array}{l}\langle x\rangle_{u^{+}-d^{+}},\left\langle x^{2}\right\rangle_{u^{--} d^{-}},\left\langle x^{3}\right\rangle_{u^{+-}-d^{+}} \\
g_{A},\left\langle x^{2}\right\rangle_{\Delta u^{+}-\Delta d^{+}}\end{array}$ & quenched & Wilson & one loop \\
\hline
\end{tabular}

\footnotetext{
* Conference proceedings.

$\dagger$ The moment $\left\langle x^{2}\right\rangle_{u-d}=A_{30}^{u-d}(0)$ is plotted in the ratio of form factors $A_{30}(t) / A_{10}(t)$, where we can use $A_{10}^{u-d}(0)=1$. The moment $\left\langle x^{2}\right\rangle_{\Delta u-\Delta d}=\tilde{A}_{30}^{u-d}(0)$ is plotted in the ratio of form factors $\tilde{A}_{30}(t) / \tilde{A}_{10}(t)$ and we can use $\tilde{A}_{10}^{u-d}(0)=g_{A}$.
}

Table B.10: Full details of lattice-QCD calculations of higher moments of unpolarized and polarized PDFs. 


\begin{tabular}{lccccccc}
\hline Mom. & NNPDF3.1 & CT14 & MMHT14 & ABMP16 & CJ15 & HERAPDF2.0 & PDF4LHC15 \\
\hline$\left\langle x^{2}\right\rangle_{u^{-}}$ & $0.0851(27)$ & $0.0841(13)$ & $0.0831(14)$ & $0.0845(8)$ & $0.0853(3)$ & $0.0886(29)$ & $0.0833(15)$ \\
$\left\langle x^{2}\right\rangle_{d^{-}}$ & $0.0284(27)$ & $0.0295(10)$ & $0.0305(11)$ & $0.0267(7)$ & $0.0305(3)$ & $0.0334(18)$ & $0.0305(17)$ \\
$\left\langle x^{2}\right\rangle_{s^{-}}$ & $0.0010(31)$ & - & $0.0006(8)$ & - & - & - & $0.0011(11)$ \\
$\left\langle x^{2}\right\rangle_{u^{-}-d^{-}}$ & $0.0571(27)$ & $0.0546(19)$ & $0.0526(19)$ & $0.0578(9)$ & $0.0548(3)$ & $0.0553(17)$ & $0.0530(24)$ \\
\hline
\end{tabular}

Table C.1: Second moments of unpolarized valence PDFs from global PDF fits at $\mu^{2}=Q^{2}=4 \mathrm{GeV}^{2}$.

\begin{tabular}{lccc}
\hline Mom. & NNPDFpol1.1 & DSSV08 & JAM17 \\
\hline$\langle x\rangle_{\Delta u^{-}}$ & $0.1493(85)$ & $0.1624(56)$ & $0.181(14)$ \\
$\langle x\rangle_{\Delta d^{-}}$ & $-0.0468(79)$ & $-0.0410(55)$ & $-0.060(18)$ \\
\hline
\end{tabular}

Table C.2: First moments of polarized valence PDFs from global PDF fits at $\mu^{2}=Q^{2}=4 \mathrm{GeV}^{2}$.

\begin{tabular}{lcccc}
\hline Mom. & NNPDFpol1.1 & DSSV14 & JAM15 & JAM17 \\
\hline$\langle 1\rangle_{\Delta g}^{\left[10^{-5}, 1\right]}$ & $-0.1(1.7)$ & $0.27\left(_{-1.44}^{+1.72}\right)$ & $1.08(87)$ & $0.18(28)$ \\
$\langle 1\rangle_{\Delta g}^{\left[10^{-3}, 1\right]}$ & $0.14(78)$ & $0.27\left(_{-0.54}^{+0.63}\right)$ & $0.74(40)$ & $0.20(24)$ \\
$\langle 1\rangle_{\Delta g}^{\left.110^{-2}, 1\right]}$ & $0.23(24)$ & $0.24\left(_{-0.15}^{+0.23}\right)$ & $0.52(19)$ & $0.18(20)$ \\
\hline
\end{tabular}

Table C.3: Truncated zeroth moments of the polarized gluon PDF from global fits at $\mu^{2}=Q^{2}=4 \mathrm{GeV}^{2}$.

\section{PDF fit results for higher moments}

In this appendix, we summarize the current values of the higher moments of unpolarized and polarized PDFs from global fits, not previously listed in Sec. 3.1.3. Even though these moments were not selected for the benchmark exercise performed in Sec. 3.2, we find it useful to collect them here for future reference.

- In Table C.1 we display the values of the second moments of the unpolarized quark valence distributions $\left\langle x^{2}\right\rangle_{u^{-}},\left\langle x^{2}\right\rangle_{d^{-}},\left\langle x^{2}\right\rangle_{s^{-}}$and $\left\langle x^{2}\right\rangle_{u^{-}-d^{-}}$.

- In Table C.2 we display the values of the first moments of the polarized quark valence distributions $\langle x\rangle_{\Delta u^{-}},\langle x\rangle_{\Delta d^{-}}$and $\langle x\rangle_{\Delta s^{-}}$.

- In Table C.3 we display the value of the zeroth moment of the polarized gluon PDF truncated in the region $\left[x_{\min }, x_{\max }\right],\langle 1\rangle_{\Delta g}^{\left[x_{\min }, x_{\max }\right]}=\int_{x_{\min }}^{x_{\max }} d x \Delta g\left(x, Q^{2}\right)$. The truncated moment is shown instead of the full moment because the latter is potentially affected by a large extrapolation uncertainty difficult to quantify (see Sec. 2.3.3). Three different truncated ranges are considered: $\left[10^{-5}, 1\right]$, $\left[10^{-3}, 1\right]$ and $\left[10^{-2}, 1\right]$.

All values in Tables C.1-C.3 are computed at $\mu^{2}=Q^{2}=4 \mathrm{GeV}^{2}$. For the description of the corresponding PDF sets and their uncertainties, see Secs. 2.3.2, 2.3.3 and 3.1.3. 


\section{References}

[1] E. Perez and E. Rizvi Rep.Prog.Phys. 76 (2013) 046201, [arXiv:1208.1178].

[2] A. De Roeck and R. S. Thorne Prog.Part.Nucl.Phys. 66 (2011) 727, [arXiv:1103.0555].

[3] S. Alekhin et al. arXiv:1101.0536.

[4] R. D. Ball et al. JHEP 04 (2013) 125, [arXiv: 1211.5142].

[5] S. Forte and G. Watt Ann. Rev. Nucl. Part. Sci. 63 (2013) 291-328, [arXiv:1301.6754].

[6] P. Jimenez-Delgado, W. Melnitchouk, and J. F. Owens J. Phys. G40 (2013) 093102, [arXiv:1306.6515].

[7] J. Rojo et al. J. Phys. G42 (2015) 103103, [arXiv:1507.00556].

[8] J. Butterworth et al. J. Phys. G43 (2016) 023001, [arXiv:1510.03865].

[9] A. Accardi et al. Eur. Phys. J. C76 (2016), no. 8 471, [arXiv:1603.08906].

[10] J. Gao, L. Harland-Lang, and J. Rojo arXiv:1709.04922.

[11] NNPDF Collaboration, R. D. Ball et al. Eur. Phys. J. C77 (2017), no. 10 663, [arXiv: 1706.00428].

[12] L. A. Harland-Lang, A. D. Martin, P. Motylinski, and R. S. Thorne Eur. Phys. J. C75 (2015), no. 5 204, [arXiv:1412.3989].

[13] S. Dulat, T.-J. Hou, J. Gao, M. Guzzi, J. Huston, P. Nadolsky, J. Pumplin, C. Schmidt, D. Stump, and C. P. Yuan Phys. Rev. D93 (2016), no. 3 033006, [arXiv:1506.07443].

[14] S. Alekhin, J. Blümlein, S. Moch, and R. Placakyte Phys. Rev. D96 (2017), no. 1014011 , [arXiv: 1701.05838].

[15] A. Accardi, L. T. Brady, W. Melnitchouk, J. F. Owens, and N. Sato Phys. Rev. D93 (2016), no. 11 114017, [arXiv:1602.03154].

[16] NNPDF Collaboration, E. R. Nocera, R. D. Ball, S. Forte, G. Ridolfi, and J. Rojo Nucl. Phys. B887 (2014) 276-308, [arXiv: 1406.5539].

[17] D. de Florian, R. Sassot, M. Stratmann, and W. Vogelsang Phys. Rev. D80 (2009) 034030, [arXiv:0904.3821].

[18] Jefferson Lab Angular Momentum Collaboration, N. Sato, W. Melnitchouk, S. E. Kuhn, J. J. Ethier, and A. Accardi Phys. Rev. D93 (2016), no. 7 074005, [arXiv: 1601.07782].

[19] Asymmetry Analysis Collaboration, M. Hirai and S. Kumano Nucl. Phys. B813 (2009) 106-122, [arXiv:0808.0413].

[20] ZEUS, H1 Collaboration, H. Abramowicz et al. Eur. Phys. J. C75 (2015), no. 12 580, [arXiv: 1506.06042].

[21] PROSA Collaboration, O. Zenaiev et al. Eur. Phys. J. C75 (2015), no. 8396 , [arXiv: 1503.04581]. 
[22] R. Gauld and J. Rojo Phys. Rev. Lett. 118 (2017), no. 7 072001, [arXiv:1610.09373].

[23] R. Boughezal, A. Guffanti, F. Petriello, and M. Ubiali JHEP 07 (2017) 130, [arXiv: 1705.00343].

[24] J. Currie, E. W. N. Glover, and J. Pires Phys. Rev. Lett. 118 (2017), no. 7072002 , [arXiv: 1611.01460].

[25] M. Czakon, N. P. Hartland, A. Mitov, E. R. Nocera, and J. Rojo JHEP 04 (2017) 044, [arXiv: 1611.08609].

[26] M. Guzzi, K. Lipka, and S.-O. Moch JHEP 01 (2015) 082, [arXiv:1406.0386].

[27] D. de Florian, R. Sassot, M. Stratmann, and W. Vogelsang Phys. Rev. Lett. 113 (2014), no. 1 012001, [arXiv: 1404.4293].

[28] Particle Data Group Collaboration, C. Patrignani et al. Chin. Phys. C40 (2016), no. 10 100001.

[29] R. Gupta in Probing the standard model of particle interactions. Proceedings, Summer School in Theoretical Physics, NATO Advanced Study Institute, 68th session, Les Houches, France, July 28-September 5, 199\%. Pt. 1, 2, pp. 83-219, 1997. hep-lat/9807028.

[30] M. Constantinou PoS LATTICE2014 (2015) 001, [arXiv: 1411.0078].

[31] S. Syritsyn PoS LATTICE2013 (2014) 009, [arXiv: 1403.4686].

[32] H.-W. Lin PoS LATTICE2012 (2012) 013, [arXiv: 1212.6849].

[33] H.-W. Lin, J.-W. Chen, S. D. Cohen, and X. Ji Phys. Rev. D91 (2015) 054510, [arXiv:1402.1462].

[34] C. Alexandrou, K. Cichy, V. Drach, E. Garcia-Ramos, K. Hadjiyiannakou, K. Jansen, F. Steffens, and C. Wiese Phys. Rev. D92 (2015) 014502, [arXiv: 1504.07455].

[35] J.-W. Chen, S. D. Cohen, X. Ji, H.-W. Lin, and J.-H. Zhang Nucl. Phys. B911 (2016) 246-273, [arXiv: 1603.06664].

[36] C. Alexandrou, K. Cichy, M. Constantinou, K. Hadjiyiannakou, K. Jansen, F. Steffens, and C. Wiese Phys. Rev. D96 (2017), no. 1 014513, [arXiv:1610.03689].

[37] R. K. Ellis, W. J. Stirling, and B. R. Webber, QCD and collider physics. Cambridge University Press, 1996.

[38] E. Leader, Spin in particle physics. Cambridge University Press, 2001.

[39] J. Collins, Foundations of perturbative QCD. Cambridge University Press, 2013.

[40] T. DeGrand and C. E. De Tar, Lattice methods for quantum chromodynamics. 2006.

[41] C. Gattringer and C. B. Lang, Quantum chromodynamics on the lattice. Springer, 2010.

[42] D. J. Gross and F. Wilczek Phys. Rev. D8 (1973) 3633-3652.

[43] D. J. Gross and F. Wilczek Phys. Rev. Lett. 30 (1973) 1343-1346. 
[44] D. J. Gross and F. Wilczek Phys. Rev. D9 (1974) 980-993.

[45] H. D. Politzer Phys. Rept. 14 (1974) 129-180.

[46] J. C. Collins and D. E. Soper Ann. Rev. Nucl. Part. Sci. 37 (1987) 383-409.

[47] J. C. Collins, D. E. Soper, and G. F. Sterman Adv. Ser. Direct. High Energy Phys. 5 (1989) 1-91, [hep-ph/0409313].

[48] J. M. Campbell, J. W. Huston, and W. J. Stirling Rept. Prog. Phys. 70 (2007) 89, [hep-ph/0611148].

[49] J. Gasser and H. Leutwyler Annals Phys. 158 (1984) 142.

[50] E. Leader and C. Lorcé Phys. Rept. 541 (2014), no. 3 163-248, [arXiv: 1309.4235].

[51] G. 't Hooft Nucl. Phys. B61 (1973) 455-468.

[52] S. Weinberg Phys. Rev. D8 (1973) 3497-3509.

[53] A. V. Manohar in Lake Louise Winter Institute: Symmetry and Spin in the Standard Model Lake Louise, Alberta, Canada, February 23-29, 1992, pp. 1-46, 1992. hep-ph/9204208.

[54] M. Anselmino, P. Gambino, and J. Kalinowski Z. Phys. C64 (1994) 267-274, [hep-ph/9401264].

[55] M. Anselmino and E. Leader Phys. Lett. B293 (1992) 216-218.

[56] J. C. Collins and D. E. Soper Nucl. Phys. B194 (1982) 445-492.

[57] G. Curci, W. Furmanski, and R. Petronzio Nucl. Phys. B175 (1980) 27-92.

[58] L. Baulieu, E. G. Floratos, and C. Kounnas Nucl. Phys. B166 (1980) 321-339.

[59] Y. L. Dokshitzer Sov. Phys. JETP 46 (1977) 641-653. [Zh. Eksp. Teor. Fiz.73,1216(1977)].

[60] V. N. Gribov and L. N. Lipatov Sov. J. Nucl. Phys. 15 (1972) 438-450. [Yad. Fiz.15,781(1972)].

[61] G. Altarelli and G. Parisi Nucl. Phys. B126 (1977) 298-318.

[62] S. Moch, J. A. M. Vermaseren, and A. Vogt Nucl. Phys. B688 (2004) 101-134, [hep-ph/0403192].

[63] A. Vogt, S. Moch, and J. A. M. Vermaseren Nucl. Phys. B691 (2004) 129-181, [hep-ph/0404111].

[64] J. Davies, A. Vogt, B. Ruijl, T. Ueda, and J. A. M. Vermaseren Nucl. Phys. B915 (2017) 335-362, [arXiv:1610.07477].

[65] S. Moch, B. Ruijl, T. Ueda, J. A. M. Vermaseren, and A. Vogt JHEP 10 (2017) 041, [arXiv: 1707.08315].

[66] S. Moch, J. A. M. Vermaseren, and A. Vogt Nucl. Phys. B889 (2014) 351-400, [arXiv: 1409.5131].

[67] A. Vogt Comput. Phys. Commun. 170 (2005) 65-92, [hep-ph/0408244].

[68] G. P. Salam and J. Rojo Comput. Phys. Commun. 180 (2009) 120-156, [arXiv:0804.3755]. 
[69] M. Botje Comput. Phys. Commun. 182 (2011) 490-532, [arXiv:1005.1481].

[70] V. Bertone, S. Carrazza, and J. Rojo Comput. Phys. Commun. 185 (2014) 1647-1668, [arXiv:1310.1394].

[71] V. Bertone, S. Carrazza, and E. R. Nocera JHEP 03 (2015) 046, [arXiv: 1501.00494].

[72] W. Giele et al. in Physics at TeV colliders. Proceedings, Euro Summer School, Les Houches, France, May 21-June 1, 2001, pp. 275-426, 2002. hep-ph/0204316.

[73] M. Dittmar et al. hep-ph/0511119.

[74] M. Di Pierro in GSA Summer School on Physics on the Frontier and in the Future Batavia, Illinois, July 31-August 7, 2000, 2000. hep-lat/0009001.

[75] G. P. Lepage in Strong interactions at low and intermediate energies. Proceedings, 13th Annual Hampton University Graduate Studies, HUGS'98, Newport News, USA, May 26-June 12, 1998, pp. 49-90, 1998. hep-lat/0506036.

[76] M. Lüscher in Probing the standard model of particle interactions. Proceedings, Summer School in Theoretical Physics, NATO Advanced Study Institute, 68th session, Les Houches, France, July 28-September 5, 1997. Pt. 1, 2, pp. 229-280, 1998. hep-lat/9802029.

[77] D. Landau and K. Binder, A Guide to Monte Carlo Simulations in Statistical Physics. Cambridge University Press, 2015.

[78] M. Newman and G. Barkema, Monte Carlo Methods in Statistical Physics. Oxford University Press, 1999.

[79] S. J. Brodsky, A. L. Deshpande, H. Gao, R. D. McKeown, C. A. Meyer, Z.-E. Meziani, R. G. Milner, J. Qiu, D. G. Richards, and C. D. Roberts arXiv:1502.05728.

[80] E.-C. Aschenauer et al. Eur. Phys. J. A53 (2017), no. 4 71, [arXiv:1410.8831].

[81] S. Aoki et al. Eur. Phys. J. C77 (2017), no. 2 112, [arXiv:1607.00299].

[82] M. Creutz Acta Phys. Slov. 61 (2011) 1-127, [arXiv:1103.3304].

[83] A. Vladikas in Modern perspectives in lattice QCD: Quantum field theory and high performance computing. Proceedings, International School, 93rd Session, Les Houches, France, August 3-28, 2009, pp. 161-222, 2011. arXiv:1103.1323.

[84] S. Chandrasekharan and U. J. Wiese Prog. Part. Nucl. Phys. 53 (2004) 373-418, [hep-lat/0405024].

[85] M. Golterman in Modern perspectives in lattice QCD: Quantum field theory and high performance computing. Proceedings, International School, 93rd Session, Les Houches, France, August 3-28, 2009, pp. 423-515, 2009. arXiv:0912.4042.

[86] M. Lüscher Commun. Math. Phys. 104 (1986) 177.

[87] M. Lüscher Commun. Math. Phys. 105 (1986) 153-188.

[88] G. P. Lepage in Boulder ASI 1989:97-120, pp. 97-120, 1989. 
[89] G. Martinelli, C. Pittori, C. T. Sachrajda, M. Testa, and A. Vladikas Nucl.Phys. B445 (1995) 81-108, [hep-lat/9411010].

[90] M. Göckeler, R. Horsley, E.-M. Ilgenfritz, H. Perlt, P. E. L. Rakow, G. Schierholz, and A. Schiller Phys. Rev. D54 (1996) 5705-5714, [hep-lat/9602029].

[91] QCDSF Collaboration, M. Göckeler, R. Horsley, D. Pleiter, P. E. L. Rakow, and G. Schierholz Phys. Rev. D71 (2005) 114511, [hep-ph/0410187].

[92] M. Lüscher JHEP 08 (2010) 071, [arXiv: 1006.4518]. [Erratum: JHEP03,092(2014)].

[93] S. Borsanyi et al. JHEP 09 (2012) 010, [arXiv: 1203.4469].

[94] S. Dürr et al. Science 322 (2008) 1224-1227, [arXiv:0906.3599].

[95] HPQCD Collaboration, I. D. Kendall, C. T. H. Davies, C. McNeile, G. P. Lepage, and J. Shigemitsu PoS LATTICE2008 (2008) 223.

[96] W. Detmold, W. Melnitchouk, and A. W. Thomas Mod.Phys.Lett. A18 (2003) 2681-2698, [hep-lat/0310003].

[97] W. Zimmermann Annals Phys. 77 (1973) 570-601. [Lect. Notes Phys.558,278(2000)].

[98] M. Göckeler, R. Horsley, E.-M. Ilgenfritz, H. Perlt, P. E. L. Rakow, G. Schierholz, and A. Schiller Phys. Rev. D53 (1996) 2317-2325, [hep-lat/9508004].

[99] J. Blümlein and H. Böttcher Phys. Lett. B662 (2008) 336-340, [arXiv:0802.0408].

[100] G. Martinelli and C. T. Sachrajda Nucl. Phys. B478 (1996) 660-686, [hep-ph/9605336].

[101] Z. Davoudi and M. J. Savage Phys. Rev. D86 (2012) 054505, [arXiv:1204.4146].

[102] C. Monahan and K. Orginos Phys. Rev. D91 (2015), no. 7 074513, [arXiv: 1501.05348].

[103] W. Detmold and C. J. D. Lin Phys. Rev. D73 (2006) 014501, [hep-lat/0507007].

[104] V. Braun and D. Mueller Eur. Phys. J. C55 (2008) 349-361, [arXiv:0709.1348].

[105] K.-F. Liu and S.-J. Dong Phys. Rev. Lett. 72 (1994) 1790-1793, [hep-ph/9306299].

[106] K.-F. Liu Phys. Rev. D62 (2000) 074501, [hep-ph/9910306].

[107] M. T. Hansen, H. B. Meyer, and D. Robaina arXiv:1704.08993.

[108] K.-F. Liu PoS LATTICE2015 (2016) 115, [arXiv: 1603.07352].

[109] K.-F. Liu Phys. Rev. D96 (2017), no. 3 033001, [arXiv:1703.04690].

[110] A. J. Chambers, R. Horsley, Y. Nakamura, H. Perlt, P. E. L. Rakow, G. Schierholz, A. Schiller, K. Somfleth, R. D. Young, and J. M. Zanotti Phys. Rev. Lett. 118 (2017), no. 24242001 , [arXiv: 1703.01153].

[111] UKQCD, QCDSF Collaboration, R. Horsley, R. Millo, Y. Nakamura, H. Perlt, D. Pleiter, P. E. L. Rakow, G. Schierholz, A. Schiller, F. Winter, and J. M. Zanotti Phys. Lett. B714 (2012) 312-316, [arXiv:1205.6410]. 
[112] QCDSF/UKQCD, CSSM Collaboration, A. J. Chambers et al. Phys. Rev. D90 (2014), no. 1 014510, [arXiv:1405.3019].

[113] A. J. Chambers et al. Phys. Rev. D92 (2015), no. 11 114517, [arXiv:1508.06856].

[114] X. Ji and C. Jung Phys. Rev. Lett. 86 (2001) 208, [hep-lat/0101014].

[115] X. Ji Phys.Rev.Lett. 110 (2013), no. 26 262002, [arXiv:1305.1539].

[116] X. Ji Sci.China Phys.Mech.Astron. 57 (2014) 1407-1412, [arXiv:1404.6680].

[117] X. Xiong, X. Ji, J.-H. Zhang, and Y. Zhao Phys. Rev. D90 (2014), no. 1014051 , $[\operatorname{arXiv}: 1310.7471]$.

[118] A. Radyushkin Phys. Lett. B767 (2017) 314-320, [arXiv:1612.05170].

[119] Y.-Q. Ma and J.-W. Qiu arXiv:1404.6860.

[120] Y.-Q. Ma and J.-W. Qiu Int. J. Mod. Phys. Conf. Ser. 37 (2015) 1560041, [arXiv:1412.2688].

[121] Y.-Q. Ma and J.-W. Qiu arXiv:1709.03018.

[122] A. V. Radyushkin Phys. Rev. D96 (2017), no. 3 034025, [arXiv:1705.01488].

[123] K. Orginos, A. Radyushkin, J. Karpie, and S. Zafeiropoulos arXiv: 1706.05373.

[124] L. Gamberg, Z.-B. Kang, I. Vitev, and H. Xing Phys. Lett. B743 (2015) 112-120, [arXiv: 1412.3401].

[125] G. S. Bali, B. Lang, B. U. Musch, and A. Schäfer Phys. Rev. D93 (2016), no. 9 094515, [arXiv: 1602 .05525].

[126] J. Green, K. Jansen, and F. Steffens arXiv:1707.07152.

[127] W. Wang, S. Zhao, and R. Zhu arXiv:1708.02458.

[128] H.-n. Li Phys. Rev. D94 (2016), no. 7 074036, [arXiv: 1602.07575].

[129] C. E. Carlson and M. Freid Phys. Rev. D95 (2017), no. 9 094504, [arXiv: 1702.05775].

[130] R. A. Briceño, M. T. Hansen, and C. J. Monahan Phys. Rev. D96 (2017), no. 1014502 , [arXiv: 1703.06072].

[131] V. S. Dotsenko and S. N. Vergeles Nucl. Phys. B169 (1980) 527-546.

[132] I. Ya. Arefeva Phys. Lett. B93 (1980) 347-353.

[133] N. S. Craigie and H. Dorn Nucl. Phys. B185 (1981) 204-220.

[134] N. G. Stefanis Nuovo Cim. A83 (1984) 205.

[135] H. Dorn Fortsch. Phys. 34 (1986) 11-56.

[136] X. Ji, J.-H. Zhang, and Y. Zhao arXiv:1706.08962.

[137] T. Ishikawa, Y.-Q. Ma, J.-W. Qiu, and S. Yoshida arXiv:1707.03107. 
[138] B. U. Musch, P. Hagler, J. W. Negele, and A. Schafer Phys. Rev. D83 (2011) 094507, [arXiv:1011.1213].

[139] T. Ishikawa, Y.-Q. Ma, J.-W. Qiu, and S. Yoshida arXiv:1609.02018.

[140] J.-W. Chen, X. Ji, and J.-H. Zhang Nucl. Phys. B915 (2017) 1-9, [arXiv:1609.08102].

[141] C. Monahan and K. Orginos JHEP 03 (2017) 116, [arXiv:1612.01584].

[142] X. Xiong, T. Luu, and U.-G. Meissner arXiv: 1705.00246.

[143] M. Constantinou and H. Panagopoulos Phys. Rev. D96 (2017), no. 5 054506, [arXiv: 1705.11193].

[144] J.-W. Chen, T. Ishikawa, L. Jin, H.-W. Lin, Y.-B. Yang, J.-H. Zhang, and Y. Zhao arXiv: 1706.01295.

[145] J.-W. Chen, T. Ishikawa, L. Jin, H.-W. Lin, Y.-B. Yang, J.-H. Zhang, and Y. Zhao arXiv: 1710.01089.

[146] M. Lüscher, P. Weisz, and U. Wolff Nucl. Phys. B359 (1991) 221-243.

[147] C. Alexandrou, K. Cichy, M. Constantinou, K. Hadjiyiannakou, K. Jansen, H. Panagopoulos, and F. Steffens Nucl. Phys. B923 (2017) 394-415, [arXiv:1706.00265].

[148] G. C. Rossi and M. Testa Phys. Rev. D96 (2017), no. 1 014507, [arXiv:1706.04428].

[149] C. Alexandrou, K. Cichy, M. Constantinou, K. Hadjiyiannakou, K. Jansen, F. Steffens, and C. Wiese PoS LATTICE2016 (2016) 151, [arXiv: 1612.08728].

[150] H.-W. Lin, J.-W. Chen, T. Ishikawa, and J.-H. Zhang arXiv:1708.05301.

[151] C. Alexandrou, S. Bacchio, K. Cichy, M. Constantinou, K. Hadjiyiannakou, K. Jansen, G. Koutsou, A. Scapellato, and F. Steffens in 35th International Symposium on Lattice Field Theory (Lattice 2017) Granada, Spain, June 18-24, 2017, 2017. arXiv:1710.06408.

[152] B. L. Ioffe Phys. Lett. 30B (1969) 123-125.

[153] V. Braun, P. Gornicki, and L. Mankiewicz Phys. Rev. D51 (1995) 6036-6051, [hep-ph/9410318].

[154] X. Ji, J.-H. Zhang, and Y. Zhao Nucl. Phys. B924 (2017) 366-376, [arXiv:1706.07416].

[155] A. V. Radyushkin arXiv:1710.08813.

[156] J. Karpie, K. Orginos, A. Radyushkin, and S. Zafeiropoulos arXiv:1710.08288.

[157] M. Stratmann and W. Vogelsang Phys. Rev. D64 (2001) 114007, [hep-ph/0107064].

[158] P. M. Nadolsky and C. P. Yuan Nucl. Phys. B666 (2003) 3-30, [hep-ph/0304001].

[159] J. C. Collins, F. Wilczek, and A. Zee Phys. Rev. D18 (1978) 242.

[160] S. J. Brodsky and G. R. Farrar Phys. Rev. Lett. 31 (1973) 1153-1156.

[161] R. D. Ball, E. R. Nocera, and J. Rojo Eur. Phys. J. C76 (2016), no. 7 383, [arXiv: 1604.00024].

[162] E. R. Nocera Phys.Lett. B742 (2015) 117-125, [arXiv:1410.7290]. 
[163] S. Forte, L. Garrido, J. I. Latorre, and A. Piccione JHEP 05 (2002) 062, [hep-ph/0204232].

[164] NNPDF Collaboration, L. Del Debbio, S. Forte, J. I. Latorre, A. Piccione, and J. Rojo JHEP 03 (2005) 080, [hep-ph/0501067].

[165] J. J. Ethier, N. Sato, and W. Melnitchouk Phys. Rev. Lett. 119 (2017), no. 13132001 , [arXiv: 1705.05889].

[166] S. J. Brodsky, A. Kusina, F. Lyonnet, I. Schienbein, H. Spiesberger, and R. Vogt Adv. High Energy Phys. 2015 (2015) 231547, [arXiv: 1504.06287].

[167] NNPDF Collaboration, R. D. Ball, V. Bertone, M. Bonvini, S. Carrazza, S. Forte, A. Guffanti, N. P. Hartland, J. Rojo, and L. Rottoli Eur. Phys. J. C76 (2016), no. 11647 , [arXiv: 1605.06515].

[168] T.-J. Hou, S. Dulat, J. Gao, M. Guzzi, J. Huston, P. Nadolsky, C. Schmidt, J. Winter, K. Xie, and C. P. Yuan arXiv:1707.00657.

[169] J. Gao, M. Guzzi, J. Huston, H.-L. Lai, Z. Li, P. Nadolsky, J. Pumplin, D. Stump, and C. P. Yuan Phys. Rev. D89 (2014), no. 3 033009, [arXiv:1302.6246].

[170] NNPDF Collaboration, R. D. Ball, L. Del Debbio, S. Forte, A. Guffanti, J. I. Latorre, J. Rojo, and M. Ubiali JHEP 05 (2010) 075, [arXiv:0912.2276].

[171] G. D’Agostini, Bayesian reasoning in data analysis: A critical introduction. 2003.

[172] G. D’Agostini Nucl. Instrum. Meth. A346 (1994) 306-311.

[173] D. Stump, J. Pumplin, R. Brock, D. Casey, J. Huston, J. Kalk, H. L. Lai, and W. K. Tung Phys. Rev. D65 (2001) 014012, [hep-ph/0101051].

[174] J. Pumplin, D. Stump, R. Brock, D. Casey, J. Huston, J. Kalk, H. L. Lai, and W. K. Tung Phys. Rev. D65 (2001) 014013, [hep-ph/0101032].

[175] J. Pumplin, D. R. Stump, J. Huston, H. L. Lai, P. M. Nadolsky, and W. K. Tung JHEP 07 (2002) 012, [hep-ph/0201195].

[176] W. T. Giele and S. Keller Phys. Rev. D58 (1998) 094023, [hep-ph/9803393].

[177] W. T. Giele, S. A. Keller, and D. A. Kosower hep-ph/0104052.

[178] G. Watt and R. S. Thorne JHEP 08 (2012) 052, [arXiv: 1205.4024].

[179] T.-J. Hou et al. JHEP 03 (2017) 099, [arXiv:1607.06066].

[180] J. Gao and P. Nadolsky JHEP 07 (2014) 035, [arXiv:1401.0013].

[181] S. Carrazza, S. Forte, Z. Kassabov, J. I. Latorre, and J. Rojo Eur. Phys. J. C75 (2015), no. 8 369, [arXiv:1505.06736].

[182] SM and NLO Multileg Working Group Collaboration, T. Binoth et al. in Physics at TeV colliders. Proceedings, 6th Workshop, dedicated to Thomas Binoth, Les Houches, France, June 8-26, 2009, pp. 21-189, 2010. arXiv:1003.1241.

[183] R. S. Thorne Phys. Rev. D86 (2012) 074017, [arXiv:1201.6180]. 
[184] A. D. Martin, R. G. Roberts, W. J. Stirling, and R. S. Thorne Eur. Phys. J. C35 (2004) 325-348, [hep-ph/0308087].

[185] A. Accardi, M. E. Christy, C. E. Keppel, P. Monaghan, W. Melnitchouk, J. G. Morfin, and J. F. Owens Phys. Rev. D81 (2010) 034016, [arXiv:0911.2254].

[186] A. D. Martin, W. J. Stirling, R. S. Thorne, and G. Watt Eur. Phys. J. C63 (2009) 189-285, [arXiv:0901.0002].

[187] NNPDF Collaboration, R. D. Ball, L. Del Debbio, S. Forte, A. Guffanti, J. I. Latorre, A. Piccione, J. Rojo, and M. Ubiali Nucl. Phys. B823 (2009) 195-233, [arXiv:0906.1958].

[188] A. D. Martin, A. J. T. M. Mathijssen, W. J. Stirling, R. S. Thorne, B. J. A. Watt, and G. Watt Eur. Phys. J. C73 (2013), no. 2 2318, [arXiv:1211.1215].

[189] A. Accardi, W. Melnitchouk, J. F. Owens, M. E. Christy, C. E. Keppel, L. Zhu, and J. G. Morfin Phys. Rev. D84 (2011) 014008, [arXiv:1102.3686].

[190] A. Manohar, P. Nason, G. P. Salam, and G. Zanderighi Phys. Rev. Lett. 117 (2016), no. 24 242002, [arXiv:1607.04266].

[191] A. V. Manohar, P. Nason, G. P. Salam, and G. Zanderighi arXiv:1708.01256.

[192] J. M. Campbell, R. K. Ellis, and C. Williams Phys. Rev. Lett. 118 (2017), no. 22 222001, [arXiv: 1612.04333].

[193] M. Czakon, D. Heymes, and A. Mitov JHEP 04 (2017) 071, [arXiv: 1606.03350].

[194] Y. Li and F. Petriello Phys. Rev. D86 (2012) 094034, [arXiv:1208.5967].

[195] T. Carli, D. Clements, A. Cooper-Sarkar, C. Gwenlan, G. P. Salam, F. Siegert, P. Starovoitov, and M. Sutton Eur. Phys. J. C66 (2010) 503-524, [arXiv:0911.2985].

[196] fastNLO Collaboration, M. Wobisch, D. Britzger, T. Kluge, K. Rabbertz, and F. Stober arXiv:1109.1310.

[197] V. Bertone, R. Frederix, S. Frixione, J. Rojo, and M. Sutton JHEP 08 (2014) 166, [arXiv: 1406.7693].

[198] J. F. Owens, A. Accardi, and W. Melnitchouk Phys. Rev. D87 (2013), no. 9 094012, [arXiv:1212.1702].

[199] S. Carrazza, A. Ferrara, D. Palazzo, and J. Rojo J. Phys. G42 (2015), no. 5 057001, [arXiv:1410.5456].

[200] S. Carrazza, J. I. Latorre, J. Rojo, and G. Watt Eur. Phys. J. C75 (2015) 474, [arXiv: 1504.06469].

[201] S. Carrazza, S. Forte, Z. Kassabov, and J. Rojo Eur. Phys. J. C76 (2016), no. 4 205, [arXiv: 1602.00005].

[202] G. Altarelli, S. Forte, and G. Ridolfi Nucl. Phys. B534 (1998) 277-296, [hep-ph/9806345].

[203] N. Cabibbo, E. C. Swallow, and R. Winston Ann. Rev. Nucl. Part. Sci. 53 (2003) 39-75, [hep-ph/0307298]. 
[204] R. Flores-Mendieta, E. E. Jenkins, and A. V. Manohar Phys. Rev. D58 (1998) 094028, [hep-ph/9805416].

[205] D. de Florian, R. Sassot, M. Epele, R. J. Hernández-Pinto, and M. Stratmann Phys. Rev. D91 (2015), no. 1 014035, [arXiv: 1410.6027].

[206] D. de Florian, M. Epele, R. J. Hernández-Pinto, R. Sassot, and M. Stratmann Phys. Rev. D95 (2017), no. 9 094019, [arXiv:1702.06353].

[207] M. Hirai, H. Kawamura, S. Kumano, and K. Saito PTEP 2016 (2016), no. 11 113B04, [arXiv: 1608.04067].

[208] N. Sato, J. J. Ethier, W. Melnitchouk, M. Hirai, S. Kumano, and A. Accardi Phys. Rev. D94 (2016), no. 11 114004, [arXiv:1609.00899].

[209] NNPDF Collaboration, V. Bertone, S. Carrazza, N. P. Hartland, E. R. Nocera, and J. Rojo Eur. Phys. J. C77 (2017), no. 8 516, [arXiv:1706.07049].

[210] I. Borsa, R. Sassot, and M. Stratmann arXiv:1708.01630.

[211] E.-C. Aschenauer et al. arXiv:1501.01220.

[212] C. Bourrely and J. Soffer Phys. Lett. B314 (1993) 132-138.

[213] C. Bourrely, J. P. Guillet, and J. Soffer Nucl. Phys. B361 (1991) 72-92.

[214] J. Dudek et al. Eur. Phys. J. A48 (2012) 187, [arXiv:1208.1244].

[215] A. Accardi et al. Eur. Phys. J. A52 (2016), no. 9 268, [arXiv:1212.1701].

[216] E. C. Aschenauer et al. arXiv:1409.1633.

[217] F. Taghavi-Shahri, H. Khanpour, S. Atashbar Tehrani, and Z. Alizadeh Yazdi Phys. Rev. D93 (2016), no. 11 114024, [arXiv:1603.03157].

[218] H. Khanpour, S. T. Monfared, and S. Atashbar Tehrani Phys. Rev. D95 (2017), no. 7 074006, [arXiv: 1703.09209].

[219] E. Nocera and S. Pisano PoS DIS2016 (2016) 284, [arXiv: 1608.08575].

[220] D. de Florian, R. Sassot, M. Stratmann, and W. Vogelsang Phys. Rev. Lett. 101 (2008) 072001, [arXiv:0804.0422].

[221] E. Leader, A. V. Sidorov, and D. B. Stamenov Phys. Rev. D82 (2010) 114018, [arXiv:1010.0574].

[222] J. Blümlein and H. Böttcher Nucl. Phys. B841 (2010) 205-230, [arXiv:1005.3113].

[223] C. Bourrely and J. Soffer Phys. Lett. B740 (2015) 168-171, [arXiv:1408.7057].

[224] E. R. Nocera, Unbiased spin-dependent Parton Distribution Functions. PhD thesis, Milan U., 2014. arXiv:1403.0440.

[225] NNPDF Collaboration, R. D. Ball, S. Forte, A. Guffanti, E. R. Nocera, G. Ridolfi, and J. Rojo Nucl. Phys. B874 (2013) 36-84, [arXiv:1303.7236]. 
[226] STAR Collaboration, L. Adamczyk et al. Phys. Rev. Lett. 115 (2015), no. 9092002 , [arXiv: 1405.5134].

[227] PHENIX Collaboration Collaboration, A. Adare et al. Phys.Rev. D90 (2014), no. 1 012007-, [arXiv:1402.6296].

[228] STAR Collaboration Collaboration, L. Adamczyk et al. Phys.Rev. D86 (2012) 032006-, [arXiv:1205.2735].

[229] PHENIX Collaboration Collaboration, A. Adare et al. Phys.Rev. D84 (2011) 012006-, [arXiv: 1009.4921].

[230] STAR Collaboration Collaboration, L. Adamczyk et al. Phys.Rev.Lett. 113 (2014) 072301-, [arXiv: 1404.6880].

[231] NNPDF Collaboration, R. D. Ball, V. Bertone, F. Cerutti, L. Del Debbio, S. Forte, A. Guffanti, J. I. Latorre, J. Rojo, and M. Ubiali Nucl. Phys. B849 (2011) 112-143, [arXiv:1012.0836]. [Erratum: Nucl. Phys.B855,927(2012)].

[232] E. R. Nocera PoS DIS2014 (2014) 204.

[233] J. Bartels, B. I. Ermolaev, and M. G. Ryskin Z. Phys. C70 (1996) 273-280, [hep-ph/9507271].

[234] J. Bartels, B. I. Ermolaev, and M. G. Ryskin Z. Phys. C72 (1996) 627-635, [hep-ph/9603204].

[235] Y. V. Kovchegov, D. Pitonyak, and M. D. Sievert JHEP 01 (2016) 072, [arXiv:1511.06737]. [Erratum: JHEP10,148(2016)].

[236] Y. V. Kovchegov, D. Pitonyak, and M. D. Sievert Phys. Rev. Lett. 118 (2017), no. 5 052001, [arXiv: 1610.06188].

[237] Y. V. Kovchegov, D. Pitonyak, and M. D. Sievert Phys. Rev. D95 (2017), no. 1 014033, [arXiv: 1610.06197].

[238] Y. V. Kovchegov, D. Pitonyak, and M. D. Sievert Phys. Lett. B772 (2017) 136-140, [arXiv: 1703.05809].

[239] Y. V. Kovchegov, D. Pitonyak, and M. D. Sievert arXiv:1706.04236.

[240] E. Leader, A. V. Sidorov, and D. B. Stamenov Phys. Rev. D84 (2011) 014002, [arXiv:1103.5979].

[241] E. C. Aschenauer, R. Sassot, and M. Stratmann Phys. Rev. D92 (2015), no. 9 094030, [arXiv: 1509.06489].

[242] E. R. Nocera J. Phys. Conf. Ser. 678 (2016), no. 1 012030, [arXiv:1510.04248].

[243] E. R. Nocera in 22nd International Symposium on Spin Physics (SPIN 2016) Urbana, IL, USA, September 25-30, 2016, 2017. arXiv:1702.05077.

[244] E. C. Aschenauer, R. Sassot, and M. Stratmann Phys. Rev. D86 (2012) 054020, [arXiv:1206.6014].

[245] NNPDF Collaboration, R. D. Ball, S. Forte, A. Guffanti, E. R. Nocera, G. Ridolfi, and J. Rojo Phys. Lett. B728 (2014) 524-531, [arXiv:1310.0461]. 
[246] E. C. Aschenauer, T. Burton, T. Martini, H. Spiesberger, and M. Stratmann Phys. Rev. D88 (2013) 114025, [arXiv:1309.5327].

[247] J. R. Green, M. Engelhardt, S. Krieg, J. W. Negele, A. V. Pochinsky, and S. N. Syritsyn Phys. Lett. B734 (2014) 290-295, [arXiv:1209.1687].

[248] C. Alexandrou, M. Constantinou, K. Hadjiyiannakou, K. Jansen, C. Kallidonis, G. Koutsou, A. V. Avilés-Casco, and C. Wiese Phys. Rev. Lett. 119 (2017) 142002, [arXiv:1706.02973].

[249] G. S. Bali, S. Collins, B. Glä $\sigma$ le, M. Göckeler, J. Najjar, R. H. Rödl, A. Schäfer, R. W. Schiel, A. Sternbeck, and W. Söldner Phys. Rev. D90 (2014), no. 7 074510, [arXiv: 1408.6850].

[250] E. Berkowitz et al. arXiv:1704.01114.

[251] T. Bhattacharya, V. Cirigliano, S. Cohen, R. Gupta, H.-W. Lin, and B. Yoon Phys. Rev. D94 (2016), no. 5 054508, [arXiv: 1606.07049].

[252] S. Capitani, M. Della Morte, D. Djukanovic, G. M. von Hippel, J. Hua, B. Jäger, P. M. Junnarkar, H. B. Meyer, T. D. Rae, and H. Wittig arXiv:1705.06186.

[253] C. Alexandrou, M. Constantinou, K. Hadjiyiannakou, K. Jansen, C. Kallidonis, G. Koutsou, and A. Vaquero Avilés-Casco Phys. Rev. D96 (2017), no. 5 054507, [arXiv: 1705.03399].

[254] G. S. Bali, S. Collins, B. Glässle, M. Göckeler, J. Najjar, R. H. Rödl, A. Schäfer, R. W. Schiel, W. Söldner, and A. Sternbeck Phys. Rev. D91 (2015), no. 5 054501, [arXiv: 1412.7336].

[255] R. Horsley, Y. Nakamura, A. Nobile, P. E. L. Rakow, G. Schierholz, and J. M. Zanotti Phys. Lett. B732 (2014) 41-48, [arXiv:1302.2233].

[256] M. Schmelling Phys. Scripta 51 (1995) 676-679.

[257] $\chi$ QCD Collaboration, M. Gong, Y.-B. Yang, J. Liang, A. Alexandru, T. Draper, and K.-F. Liu Phys. Rev. D95 (2017), no. 11 114509, [arXiv:1511.03671].

[258] M. Engelhardt Phys. Rev. D86 (2012) 114510, [arXiv:1210.0025].

[259] Y. Aoki, T. Blum, H.-W. Lin, S. Ohta, S. Sasaki, R. Tweedie, J. Zanotti, and T. Yamazaki Phys. Rev. D82 (2010) 014501, [arXiv:1003.3387].

[260] LHPC Collaboration, J. D. Bratt et al. Phys. Rev. D 82 (2010) 094502, [arXiv:1001.3620].

[261] A. Abdel-Rehim et al. Phys. Rev. D92 (2015), no. 11 114513, [arXiv: 1507.04936]. [Erratum: Phys. Rev.D93,no.3,039904(2016)].

[262] NNPDF Collaboration, R. D. Ball et al. JHEP 04 (2015) 040, [arXiv: 1410.8849].

[263] R. D. Ball, V. Bertone, F. Cerutti, L. Del Debbio, S. Forte, A. Guffanti, N. P. Hartland, J. I. Latorre, J. Rojo, and M. Ubiali Nucl. Phys. B855 (2012) 608-638, [arXiv:1108.1758].

[264] HERAFitter developers' Team Collaboration, S. Camarda et al. Eur. Phys. J. C75 (2015), no. 9 458, [arXiv:1503.05221].

[265] H. Paukkunen and P. Zurita JHEP 12 (2014) 100, [arXiv:1402.6623].

[266] S. Alekhin et al. Eur. Phys. J. C75 (2015), no. 7 304, [arXiv:1410.4412]. 
[267] H.-W. Lin, W. Melnitchouk, A. Prokudin, N. Sato, and H. Shows arXiv: 1710.09858.

[268] A. Abdel-Rehim, C. Alexandrou, M. Constantinou, V. Drach, K. Hadjiyiannakou, K. Jansen, G. Koutsou, and A. Vaquero Phys. Rev. D89 (2014), no. 3 034501, [arXiv:1310.6339].

[269] M. Deka et al. Phys. Rev. D91 (2015), no. 1 014505, [arXiv:1312.4816].

[270] C. Alexandrou, M. Constantinou, K. Hadjiyiannakou, K. Jansen, H. Panagopoulos, and C. Wiese Phys. Rev. D96 (2017), no. 5 054503, [arXiv:1611.06901].

[271] LHPC, SESAM Collaboration, D. Dolgov et al. Phys. Rev. D66 (2002) 034506, [hep-lat/0201021].

[272] M. Deka, T. Streuer, T. Doi, S. J. Dong, T. Draper, K. F. Liu, N. Mathur, and A. W. Thomas Phys. Rev. D79 (2009) 094502, [arXiv:0811.1779].

[273] J. Green, N. Hasan, S. Meinel, M. Engelhardt, S. Krieg, J. Laeuchli, J. Negele, K. Orginos, A. Pochinsky, and S. Syritsyn Phys. Rev. D95 (2017), no. 11 114502, [arXiv:1703.06703].

[274] QCDSF Collaboration, G. S. Bali et al. Phys. Rev. Lett. 108 (2012) 222001, [arXiv:1112.3354].

[275] B. Yoon et al. Phys. Rev. D95 (2017), no. 7 074508, [arXiv:1611.07452].

[276] G. von Hippel, T. D. Rae, E. Shintani, and H. Wittig Nucl. Phys. B914 (2017) 138-159, [arXiv: 1605.00564].

[277] J. Dragos, R. Horsley, W. Kamleh, D. B. Leinweber, Y. Nakamura, P. E. L. Rakow, G. Schierholz, R. D. Young, and J. M. Zanotti Phys. Rev. D94 (2016), no. 7074505 , [arXiv: 1606.03195].

[278] Y.-B. Yang, A. Alexandru, T. Draper, M. Gong, and K.-F. Liu Phys. Rev. D93 (2016), no. 3 034503, [arXiv:1509.04616].

[279] T. Bhattacharya, S. D. Cohen, R. Gupta, A. Joseph, H.-W. Lin, and B. Yoon Phys. Rev. D89 (2014), no. 9 094502, [arXiv:1306.5435].

[280] C. Alexandrou, M. Constantinou, S. Dinter, V. Drach, K. Jansen, C. Kallidonis, and G. Koutsou Phys. Rev. D88 (2013), no. 1 014509, [arXiv:1303.5979].

[281] B. J. Owen, J. Dragos, W. Kamleh, D. B. Leinweber, M. S. Mahbub, B. J. Menadue, and J. M. Zanotti Phys. Lett. B723 (2013) 217-223, [arXiv:1212.4668].

[282] S. Capitani, M. Della Morte, G. von Hippel, B. Jäger, A. Jüttner, B. Knippschild, H. B. Meyer, and H. Wittig Phys. Rev. D86 (2012) 074502, [arXiv:1205.0180].

[283] C. Alexandrou, J. Carbonell, M. Constantinou, P. A. Harraud, P. Guichon, K. Jansen, C. Kallidonis, T. Korzec, and M. Papinutto Phys. Rev. D83 (2011) 114513, [arXiv:1104.1600].

[284] T. Yamazaki, Y. Aoki, T. Blum, H.-W. Lin, S. Ohta, S. Sasaki, R. Tweedie, and J. Zanotti Phys. Rev. D79 (2009) 114505, [arXiv:0904.2039].

[285] RBC+UKQCD Collaboration, T. Yamazaki, Y. Aoki, T. Blum, H. W. Lin, M. F. Lin, S. Ohta, S. Sasaki, R. J. Tweedie, and J. M. Zanotti Phys. Rev. Lett. 100 (2008) 171602, [arXiv:0801.4016]. 
[286] H.-W. Lin, T. Blum, S. Ohta, S. Sasaki, and T. Yamazaki Phys. Rev. D78 (2008) 014505, [arXiv:0802.0863].

[287] LHPC Collaboration, P. Hägler et al. Phys. Rev. D77 (2008) 094502, [arXiv:0705.4295].

[288] C. Alexandrou, G. Koutsou, T. Leontiou, J. W. Negele, and A. Tsapalis Phys. Rev. D76 (2007) 094511, [arXiv:0706.3011]. [Erratum: Phys. Rev.D80,099901(2009)].

[289] LHPC Collaboration, R. G. Edwards, G. T. Fleming, P. Hägler, J. W. Negele, K. Orginos, A. V. Pochinsky, D. B. Renner, D. G. Richards, and W. Schroers Phys. Rev. Lett. 96 (2006) 052001, [hep-lat/0510062].

[290] A. A. Khan et al. Phys. Rev. D74 (2006) 094508, [hep-lat/0603028].

[291] R. Babich, R. C. Brower, M. A. Clark, G. T. Fleming, J. C. Osborn, C. Rebbi, and D. Schaich Phys. Rev. D85 (2012) 054510, [arXiv:1012.0562].

[292] SESAM Collaboration, S. Güsken, P. Ueberholz, J. Viehoff, N. Eicker, T. Lippert, K. Schilling, A. Spitz, and T. Struckmann Phys. Rev. D59 (1999) 114502, [hep-lat/9901009].

[293] S. J. Dong, J. F. Lagae, and K. F. Liu Phys. Rev. Lett. 75 (1995) 2096-2099, [hep-ph/9502334].

[294] M. Fukugita, Y. Kuramashi, M. Okawa, and A. Ukawa Phys. Rev. Lett. 75 (1995) 2092-2095, [hep-lat/9501010].

[295] R. Gupta and J. E. Mandula Phys. Rev. D50 (1994) 6931-6938, [hep-lat/9402018].

[296] B. Allés, M. Campostrini, L. Del Debbio, A. Di Giacomo, H. Panagopoulos, and E. Vicari Phys. Lett. B336 (1994) 248-250, [hep-lat/9402019].

[297] R. Altmeyer, M. Göckeler, R. Horsley, E. Laermann, and G. Schierholz Phys. Rev. D49 (1994) 3087-3090.

[298] J. E. Mandula and M. C. Ogilvie Phys. Lett. B312 (1993) 327-332, [hep-lat/9208009].

[299] K.-F. Liu in Future physics with light and heavy nuclei at HERMES. Contributions to the proceedings of the '95 - '96 Workshop on Future Physics at HERA, working group on light and heavy nuclei at HERA. Part 2, 1995. hep-lat/9510046.

[300] C. Alexandrou, M. Constantinou, S. Dinter, V. Drach, K. Hadjiyiannakou, K. Jansen, G. Koutsou, and A. Vaquero JHEP 06 (2015) 068, [arXiv:1501.03734].

[301] G. S. Bali, S. Collins, M. Deka, B. Glä $\boldsymbol{\sigma l e , ~ M . ~ G o ̈ c k e l e r , ~ J . ~ N a j j a r , ~ A . ~ N o b i l e , ~ D . ~ P l e i t e r , ~}$ A. Schäfer, and A. Sternbeck Phys. Rev. D86 (2012) 054504, [arXiv:1207.1110].

[302] QCDSF/UKQCD Collaboration, D. Pleiter et al. PoS LATTICE2010 (2010) 153, [arXiv: 1101.2326].

[303] S. N. Syritsyn, J. R. Green, J. W. Negele, A. V. Pochinsky, M. Engelhardt, P. Hagler, B. Musch, and W. Schroers PoS LATTICE2011 (2011) 178, [arXiv:1111.0718].

[304] M. Göckeler, R. Horsley, L. Mankiewicz, H. Perlt, P. E. L. Rakow, G. Schierholz, and A. Schiller Phys. Lett. B414 (1997) 340-346, [hep-ph/9708270]. 
[305] M. Göckeler, R. Horsley, D. Pleiter, P. E. L. Rakow, A. Schäfer, G. Schierholz, H. Stüben, and J. M. Zanotti Phys. Rev. D72 (2005) 054507, [hep-lat/0506017].

[306] QCDSF Collaboration, M. Göckeler, P. Hägler, R. Horsley, D. Pleiter, P. E. L. Rakow, A. Schäfer, G. Schierholz, and J. M. Zanotti Nucl. Phys. Proc. Suppl. 140 (2005) 399-404, [hep-lat/0409162]. [,399(2004)].

[307] M. Göckeler, R. Horsley, W. Kürzinger, H. Oelrich, D. Pleiter, P. E. L. Rakow, A. Schäfer, and G. Schierholz Phys. Rev. D63 (2001) 074506, [hep-lat/0011091]. 